\title{
THE REGULARIZED SIEGEL-WEIL FORMULA (THE SECOND TERM IDENTITY) AND THE RALLIS INNER PRODUCT FORMULA
}

\author{
WEE TECK GAN, YANNAN QIU AND SHUICHIRO TAKEDA
}

In memory of a pioneer

Steve Rallis (1942-2012)

\begin{abstract}
In this paper, we establish the second term identity of the Siegel-Weil formula in full generality, and derive the Rallis inner product formula for global theta lifts for any dual pair. As a corollary, we resolve the non-vanishing problem of global theta lifts initiated by Steve Rallis.
\end{abstract}

\section{Introduction}

About 30 years ago, Steve Rallis ([R1, R2, R3]) initiated a program to understand the cuspidality and non-vanishing of global theta liftings. We take a few moments to describe Rallis' program and his fundamental contributions to it.

1.1. Theta correspondence. Let $F$ be a number field with the ring of adeles $\mathbb{A}$, and let $E$ be either $F$ or a quadratic extension of $F$. With $\epsilon= \pm 1$, let $U_{n}$ be an $n$-dimensional $-\epsilon$-Hermitian space over $E$, and let $V_{r}$ be an $m$-dimensional $\epsilon$-Hermitian space of Witt index $r$. Then one has an associated reductive dual pair $G\left(U_{n}\right) \times H\left(V_{r}\right)$, where $G\left(U_{n}\right)$ is the isometry group of $U_{n}$ (or a covering thereof). The group $G\left(U_{n}\right)(\mathbb{A}) \times H\left(V_{r}\right)(\mathbb{A})$ has a Weil representation $\omega$ (depending on some other auxiliary data), and one has an automorphic realization

$$
\theta: \omega \longrightarrow\left\{\text { Functions on }\left[G\left(U_{n}\right)\right] \times\left[H\left(V_{r}\right)\right]\right\}
$$

where we have written $\left[G\left(U_{n}\right)\right]$ for $G\left(U_{n}\right)(F) \backslash G\left(U_{n}\right)(\mathbb{A})$. If $\pi$ is a cuspidal automorphic representation of $G\left(U_{n}\right)(\mathbb{A})$, then the global theta lift $\Theta(\pi)$ of $\pi$ to $H\left(V_{r}\right)$ is the automorphic representation of $H\left(V_{r}\right)$ spanned by the functions

$$
\theta(\phi, f)(h)=\int_{G\left(U_{n}\right)(F) \backslash G\left(U_{n}\right)(\mathbb{A})} \theta(\phi)(g, h) \cdot \overline{f(g)} d g
$$

where $f \in \pi, \phi \in \omega$ and $d g$ is the Tamagawa measure.

The main problem in the theory of theta correspondence is to investigate the cuspidality and nonvanishing of $\Theta(\pi)$. The cuspidality issue was quickly handled by Rallis in [R2] where he discovered the so-called tower property. Thus the remaining issue is the question of nonvanishing.

1.2. Rallis' program. The goal of Rallis' program is to obtain a local-global criterion for the nonvanishing of the global theta lifting $\Theta(\pi)$, with a prototype statement:

- The global theta lifting $\Theta(\pi)$ to $H\left(V_{r}\right)(\mathbb{A})$ of a cuspidal representation $\pi$ on $G\left(U_{n}\right)(\mathbb{A})$ is nonzero if and only if

- the local theta liftings to $H\left(V_{r}\right)\left(F_{v}\right)$ of $\pi_{v}$ on $G\left(U_{n}\right)\left(F_{v}\right)$ are nonzero for all places $v$ of $F$;

2000 Mathematics Subject Classification. 11F27, 11F70, 22E50.

Key words and phrases. Siegel-Weil formula, theta correspondence, Rallis inner product. 
- the standard L-function $L(s, \pi)$ of $\pi$ is nonvanishing or has a pole at a distinguished point $s_{0}$.

Besides drawing from his earlier work ([RS1, RS2, RS3] ) with G. Schiffmann, Rallis was motivated by the then-recently-appeared results of Waldspurger [W] who proved such a result for the theta correspondence between $\mathrm{O}_{3}$ and $\mathrm{Mp}_{2}$. Moreover, Rallis was able to carry out this program for the theta correspondence between $\mathrm{O}_{n}$ and $\mathrm{Mp}_{2}$ in [R2, R3].

1.3. The Rallis Inner Product Formula. The cornerstone of Rallis' program is the so-called Rallis inner product formula. Namely, one may determine the non-vanishing of $\Theta(\pi)$ by computing the Petersson inner product $\langle\theta(\phi, f), \theta(\phi, f)\rangle$. The Rallis inner product formula relates this inner product to the $L$ values of $\pi$.

The mechanism for the Rallis inner product formula relies on the following see-saw diagram of dual pairs:

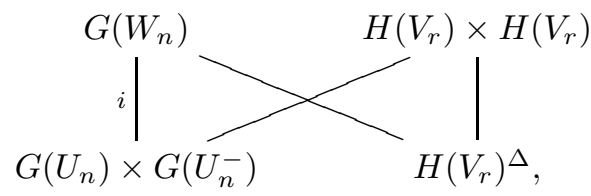

where $U_{n}^{-}$denotes the $-\epsilon$-Hermitian space obtained from $U_{n}$ by multiplying the form by -1 , so that $G\left(U_{n}^{-}\right)=G\left(U_{n}\right)$, and $W_{n}$ (to be read "doubled-U") denotes the space $U_{n}+\left(U_{n}^{-}\right)$. The resulting see-saw identity reads:

$$
\begin{aligned}
& \left\langle\theta\left(\phi_{1}, f_{1}\right), \theta\left(\phi_{2}, f_{2}\right)\right\rangle \\
= & \int_{\left[H\left(V_{r}\right)\right]}\left(\int_{\left[G\left(U_{n}\right)\right]} \theta\left(\phi_{1}\right)\left(g_{1}, h\right) \cdot \overline{f_{1}\left(g_{1}\right)} d g_{1}\right) \cdot\left(\int_{\left[G\left(U_{n}\right)\right]} \overline{\theta\left(\phi_{2}\right)\left(g_{2}, h\right)} \cdot f_{2}\left(g_{2}\right) d g_{2}\right) d h \\
= & \int_{\left[G\left(U_{n}\right) \times G\left(U_{n}\right)\right]}\left(\int_{\left[H\left(V_{r}\right)\right]} \theta\left(\phi_{1}\right)\left(g_{1}, h\right) \cdot \overline{\theta\left(\phi_{2}\right)\left(g_{2}, h\right)} d h\right) \cdot \overline{f_{1}\left(g_{1}\right)} \cdot f_{2}\left(g_{2}\right) d g_{1} d g_{2}
\end{aligned}
$$

where in the last equality, we have formally exchanged the integrals.

In order to justify the above exchange and to relate the last expression above to L-functions, Rallis was led to develop, in collaboration with S. Kudla and Piatetski-Shapiro, several key ingredients. We recall these key ingredients in turn and some recent developments concerning them.

1.4. A regularized Siegel-Weil formula. The Siegel-Weil formula was discovered by Siegel in the context of classical modular forms and then cast in the representation theoretic language and considerably extended in an influential paper of Weil [We1]. It identifies the global theta lift of the trivial representation of $H\left(V_{r}\right)$ to $G\left(W_{n}\right)$ (which is the inner integral in (1.1)) as an Eisenstein series, at least when some convergence conditions are satisfied. In a series of 3 papers [KR1, KR2, KR5], Kudla and Rallis greatly extended the theory of the Siegel-Weil formula to situations where these convergence conditions are not satisfied. Their work culminates in a regularized Siegel-Weil formula, and they established what is now known as the first term identity, at least when $G\left(W_{n}\right)$ is symplectic and $H\left(V_{r}\right)$ orthogonal. Their work was subsequently refined and extended to other dual pairs by others ([Ik, I1, I2, I3, Y2, Y3, IM0, JS]), especially in the work of Ikeda, Ichino and Yamana.

In particular, the first term identity in the so-called first term range (see below) is now completely established. Thus, one has a re-interpretation of the inner integral in (1.1) as a special value or residue of a Siegel Eisenstein series. 
1.5. The theory of the doubling zeta integral. The regularized Siegel-Weil formula led PiatetskiShapiro and Rallis [PS-R] to consider the doubling zeta integral, which is the outer integral in (1.1), with the inner integral replaced by a Siegel-Eisenstein series on $G\left(W_{n}\right)$. This is a family of zeta integrals giving rise, à la Tate's thesis and Godement-Jacquet GJ], to a theory of the standard L-function $L(s, \pi)$ for classical groups. In a paper of Lapid-Rallis [LR, the local theory of the doubling zeta integral was worked out in full detail. In particular, the local standard $\gamma$-factor (twisted by $\mathrm{GL}_{1}$ ) was precisely defined, and characterized by a list of properties (the Ten Commandments). Moreover, the local L-factor and $\epsilon$-factor were defined from the $\gamma$-factor following a procedure of Shahidi. These local L-factors and $\epsilon$-factors are the "right" ones, in the sense that they are compatible with those on Galois side under the local Langlands correspondence.

In a recent paper [Y4, Yamana showed that the local L-factors defined by Lapid-Rallis [LR] are precisely the GCD's for the local zeta integrals associated to the family of "good" sections. This implies that the analytic properties of the local zeta integrals are precisely controlled by the standard L-factors. With this, the theory of the doubling zeta integral is essentially complete.

Globally, the study of the doubling zeta integral leads to a precise understanding of the analytic properties of the standard L-functions of classical groups, such as the possible location of poles of these L-functions ( KR3, T2, Y4]).

1.6. Local theta correspondence. In the course of establishing the Siegel-Weil formula and in the application of the theory of the doubling zeta integrals, Kudla and Rallis resolved many of the local problems in the theory of the local theta correspondence. In a series of papers [KR3, KR4, KR6, KS, S1, S2, they, together with Sweet, completely determined the structure of certain degenerate principal series representations and described their constituents in the framework of local theta correspondence in the $p$-adic case. Their work partly motivated and was complemented by a similar analysis in the archimedean case, which was carried out in L1, L2, HL, LZ1, LZ2, LZ3. This, together with their study of the local doubling zeta integral, led Kudla and Rallis to formulate a conjecture known as the conservation relation, which is an identity for the sum of the first occurrences of local theta correspondence in two different Witt towers. In [KR6], they made a key progress towards this conjecture by proving one inequality of this identity. The reverse inequality was shown in a recent preprint [SZ of Sun and Zhu, thus completing the proof of the conservation conjecture.

1.7. First Term Range. The above developments imply that the local issues involved in the Rallis' program are completely settled (except for a certain subtle issue for some of the real cases, which will be discussed in the final section of this paper). In particular, the above achievements culminate in a Rallis inner product formula for the theta lift from $G\left(U_{n}\right)$ to $H\left(V_{r}\right)$ in the following cases:

- (Weil's convergent range) $r=0$ or $m-r>n+\epsilon_{0}$ ( KR1, Li, I3, Y4]);

- (First term range) $r>0$ and $m \leq n+\epsilon_{0}$, so that $m-r \leq n+\epsilon_{0}$, ([KR5, GT, Y4]),

where we define

$$
\epsilon_{0}= \begin{cases}0, & \text { if } E \neq F \\ \epsilon, \text { if } E=F\end{cases}
$$

In the first term range, the Rallis inner product formula takes the rough form:

$$
\left\langle\theta\left(\phi_{1}, f_{1}\right), \theta\left(\phi_{2}, f_{2}\right)\right\rangle=c \cdot \operatorname{Res}_{s=-s_{0}} L\left(s+\frac{1}{2}, \pi\right) \cdot Z^{*}\left(-s_{0}, \phi_{1} \otimes \overline{\phi_{2}}, f_{1}, f_{2}\right)
$$

where

$$
-s_{0}=-\frac{m-\left(n+\epsilon_{0}\right)}{2} \geq 0
$$


$c$ is some explicit nonzero constant, and $Z^{*}$ denotes the normalized global doubling zeta integral. Here, when $s_{0}=0$, one actually has the value $L\left(\frac{1}{2}, \pi\right)$ rather than the residue of $L\left(s+\frac{1}{2}, \pi\right)$ at $s=0$.

Using this, Yamana obtained in [Y4] the local-global criterion for the nonvanishing of the global theta lifting in the first term range, thus completing Rallis' program when $m \leq n+\epsilon_{0}$.

1.8. Purpose of this paper. After the above, one is left with the case when

$$
r>0, \quad n+\epsilon_{0}<m \leq\left(n+\epsilon_{0}\right)+r .
$$

In this case, one expects to have a Rallis inner product formula involving the value of $L(s, \pi)$ at $s=s_{0}>0$. For this purpose, it turns out that one needs to show a second term identity of the regularized Siegel-Weil formula.

The purpose of this paper is to supply this remaining global ingredient, i.e. we will prove the general second term identity of the regularized Siegel-Weil formula, thereby obtaining the Rallis inner product formula in complete generality.

We note that special cases of the second term identity have been known for some time. It was shown in [KRS] when $\left(H\left(V_{r}\right), G\left(W_{n}\right)\right)=\left(\mathrm{O}_{4}, \mathrm{Sp}_{4}\right)$ and in [T1 for $\left(\mathrm{U}_{3}, \mathrm{U}_{4}\right)$. In addition, when the groups $H\left(V_{r}\right)$ and $G\left(W_{n}\right)$ are the symplectic (resp. split orthogonal) and split orthogonal (resp. symplectic) groups, a second term identity for the spherical vector was shown in [GT] (resp. [K3]). The analogous spherical second term identity for unitary groups was shown by $\mathrm{W}$. Xiong in $\mathrm{X}$. In a recent paper $\mathrm{Q}$, the second-term identity for general vectors was proved by Y. Qiu when $H\left(V_{r}\right)$ and $G\left(W_{n}\right)$ are orthogonal and symplectic with $n=r$.

1.9. The Regularized Theta Integral. Let us give a more precise description of the results of this paper. Assume that we are outside the Weil's convergent range, so that $r>0$ and $m-r \leq n+\epsilon_{0}$, in which case we have

$$
0<m \leq 2 \cdot\left(n+\epsilon_{0}\right) \quad \text { and } \quad r \leq n+\epsilon_{0} .
$$

We shall further assume (as did Kudla-Rallis in KR5) that $r \leq n$. This is only a condition when $\epsilon=\epsilon_{0}=1$. In any case, consider the Weil representation $\omega_{n, r}$ of $G\left(W_{n}\right) \times H\left(V_{r}\right)$ and its automorphic realization $\theta_{n, r}$. We are interested in the theta integral

$$
I_{n, r}(\phi)(g)=\frac{1}{\tau\left(H\left(V_{r}\right)\right)} \cdot \int_{\left[H\left(V_{r}\right)\right]} \theta_{n, r}(\phi)(g, h) d h .
$$

This integral diverges, but under the above conditions Kudla-Rallis [KR5] discovered a regularization of this theta integral, which gives a meromorphic function $B^{n, r}(s, \phi)$. One is interested in the behavior of $B^{n, r}(s, \phi)$ at

$$
s=\rho_{H\left(V_{r}\right)}=\frac{m-r-\epsilon_{0}}{2} .
$$

It turns out that in the first term range, when $m \leq n+\epsilon_{0}, B^{n, r}(s, \phi)$ has a pole of order at most 1 (which is attained for some $\phi$ ) whereas in the second term range, when

$$
n+\epsilon_{0}<m \leq 2 \cdot\left(n+\epsilon_{0}\right),
$$

$B^{n, r}(s, \phi)$ has a pole of order at most 2. Thus, the Laurent expansion of $B^{n, r}(s, \phi)$ at $s=\rho_{H(V)}$ has the form

and

$$
B^{n, r}(s, \phi)=\frac{B_{-1}^{n, r}(\phi)}{s-\rho_{H_{r}}}+B_{0}^{n, r}(\phi)+\cdots \quad \text { in the first term range; }
$$

$$
B^{n, r}(s, \phi)=\frac{B_{-2}^{n, r}(\phi)}{\left(s-\rho_{H_{r}}\right)^{2}}+\frac{B_{-1}^{n, r}(\phi)}{s-\rho_{H_{r}}}+B_{0}^{n, r}(\phi)+\cdots \quad \text { in the second term range. }
$$


Let us note that each Laurent coefficient $B_{d}^{n, r}(\phi)$ is an automorphic form on $G\left(W_{n}\right)$, and hence we view $B_{d}^{n, r}$ as a linear map

$$
B_{d}^{n, r}: \omega_{n, r} \rightarrow \mathcal{A}\left(G\left(W_{n}\right)\right),
$$

where $\mathcal{A}\left(G\left(W_{n}\right)\right)$ is the space of automorphic forms on $G\left(W_{n}\right)$.

1.10. First Term Identity in First Term Range. The purpose of the Siegel-Weil formula is to identify the automorphic forms $B_{d}^{n, r}(\phi)$, as much as possible, with the analogous Laurent coefficients of a Siegel-Eisenstein series $A^{n, r}(s, \phi)$ associated to $\phi$ by the formation of Siegel-Weil sections at $s$ equal to

$$
s_{m, n}:=\left(m-n-\epsilon_{0}\right) / 2 .
$$

Observe that in the first term range, $s_{m, n} \leq 0$, whereas in the second term range, $s_{m, n}>0$. At $s=s_{m, n}>0$, the Laurent expansion of the Siegel-Eisenstein series $A^{n, r}(s, \phi)$ has the form

$$
A^{n, r}(s, \phi)=\frac{A_{-1}^{n, r}(\phi)}{s-s_{m, n}}+A_{0}^{n, r}(\phi)+\cdots
$$

As for $B_{d}^{n, r}$, each $A_{d}^{n, r}(\phi)$ is an automorphic form on $G\left(W_{n}\right)$ and hence $A_{d}^{n, r}$ is viewed as a linear map

$$
A_{d}^{n, r}: \omega_{n, r} \rightarrow \mathcal{A}\left(G\left(W_{n}\right)\right) .
$$

Assume that we are in the first term range, so that $m \leq n+\epsilon_{0}$ and $s_{m, n} \leq 0$. Let $r^{\prime} \geq r$ be defined by

$$
\operatorname{dim} V_{r}+\operatorname{dim} V_{r^{\prime}}=2 \cdot\left(n+\epsilon_{0}\right) .
$$

The space $V_{r^{\prime}}$ is called the complementary space to $V_{r}$ with respect to $W_{n}$ and its dimension $m^{\prime}=m_{0}+2 r^{\prime}$ is such that $s_{m^{\prime}, n} \geq 0$. Ikeda has defined in $\left[\mathrm{Ik}\right.$ a $G\left(W_{n}\right) \times H\left(V_{r}\right)$-equivariant map

$$
\mathrm{Ik}^{n, r^{\prime}}: \omega_{n, r^{\prime}} \longrightarrow \omega_{n, r}
$$

which we shall call the Ikeda map. Then the first term identity established in [KR5, Mo, JS, I2, GT, YY2] for various dual pairs is the following identity: assuming that $V_{r}$ is not the split binary quadratic space, then for all $\phi \in \omega_{n, r}$,

$$
a_{n, r^{\prime}} \cdot A_{-1}^{n, r^{\prime}}\left(\phi^{\prime}\right)=A_{0}^{n, r}(\phi)=2 \cdot B_{-1}^{n, r}(\phi),
$$

where $\phi^{\prime} \in \omega_{n, r^{\prime}}$ is such that

$$
\operatorname{Ik}^{n, r^{\prime}}\left(\pi_{K_{H_{r^{\prime}}}} \phi^{\prime}\right)=\phi
$$

$\pi_{K_{H_{r^{\prime}}}}$ is the projection onto the $K_{H_{r^{\prime}}}$-fixed space (with $K_{H_{r^{\prime}}}$ a maximal compact subgroup of $H_{r^{\prime}}(\mathbb{A})$ ) and $a_{n, r^{\prime}}$ is some nonzero explicit constant. There is an analogous statement for the split binary quadratic case. We recall these results in Theorems $7.1,7.3$ and 7.4 below.

Observe here that $A_{0}^{n, r}$ is the zeroth Laurent coefficient at $s_{m, n} \leq 0$, whereas $A_{-1}^{n, r^{\prime}}$ is the $-1^{\text {st }}$ Laurent coefficient at $s=s_{m^{\prime}, n} \geq 0$. One may interpret the identity $a_{n, r^{\prime}} \cdot A_{-1}^{n, r^{\prime}}\left(\phi^{\prime}\right)=2 \cdot B_{-1}^{n, r}(\phi)$ as saying that $A_{-1}^{n, r^{\prime}}$ and $B_{-1}^{n, r} \circ \mathrm{Ik}^{n, r^{\prime}}$ are proportional as linear maps $\omega_{n, r^{\prime}} \rightarrow \mathcal{A}\left(G\left(W_{n}\right)\right)$, and similarly for the identity $A_{0}^{n, r}(\phi)=2 \cdot B_{-1}^{n, r}(\phi)$.

1.11. The Main Results. The goal of this paper is to prove the first and second term identities in the second term range. More precisely, we show:

Theorem 1.2 (Siegel-Weil formula). Suppose that $0<r \leq n$ and $n+\epsilon_{0}<m \leq n+\epsilon_{0}+r$, so that we are in the second term range. Then one has:

(i) (First term identity) For all $\phi \in \omega_{n, r}$, one has

$$
A_{-1}^{n, r}(\phi)=B_{-2}^{n, r}(\phi)
$$


(ii) (Second term identity) For all $\phi \in \omega_{n, r}$, one has

$$
A_{0}^{n, r}(\phi)=B_{-1}^{n, r}(\phi)-\kappa_{r, r^{\prime}} \cdot\left\{B_{0}^{n, r^{\prime}}\left(\operatorname{Ik}^{n, r}\left(\pi_{K_{H_{r}}} \phi\right)\right)\right\} \quad \bmod \operatorname{Im} A_{-1}^{n, r} .
$$

Here, $\kappa_{r, r^{\prime}}>0$ is some explicit constant and $r^{\prime}<r$ is such that

$$
\operatorname{dim} V_{r}+\operatorname{dim} V_{r^{\prime}}=2 \cdot\left(n+\epsilon_{0}\right),
$$

so that $V_{r^{\prime}}$ is the complementary space to $V_{r}$ with respect to $W_{n}$. Moreover,

$$
\mathrm{Ik}^{n, r}: \omega_{n, r} \longrightarrow \omega_{n, r^{\prime}}
$$

is the Ikeda map which is $G\left(W_{n}\right) \times H\left(V_{r^{\prime}}\right)$-equivariant. Finally, the term $\{\ldots\}$ on the $R H S$ is interpreted to be 0 if $V_{r^{\prime}}$ is anisotropic or is equal to the split binary quadratic form.

Note that the equality in the second term identity in (ii) is viewed as an equality in $\mathcal{A}\left(G\left(W_{n}\right)\right) / \operatorname{Im} A_{-1}^{n, r}$ where $A_{-1}^{n, r}$ is viewed as a linear map $A_{-1}^{n, r}: \omega_{n, r} \rightarrow \mathcal{A}\left(G\left(W_{n}\right)\right)$.

The proof of this theorem is a significant refinement of the techniques of [GT] and is based on induction on the quantity:

$$
\mathcal{N}=m-\left(n+\epsilon_{0}\right)
$$

Observe that, outside the Weil's convergent range,

$$
\begin{aligned}
& \mathcal{N}<0 \Longleftrightarrow \text { first term range; } \\
& \mathcal{N}=0 \Longleftrightarrow \text { boundary case; } \\
& \mathcal{N}>0 \Longleftrightarrow \text { second term range. }
\end{aligned}
$$

To go beyond [GT], which is limited to the $G\left(W_{n}\right)$-span of the spherical vector, we need to make a more detailed study of a $G\left(W_{n}\right)(\mathbb{A}) \times H\left(V_{r}\right)(\mathbb{A})$-equivariant map $F^{n, r}(s,-)$ (see $\$ 4$ ) from the Weil representation to a family of induced representations of $G\left(W_{n}\right) \times H\left(V_{r}\right)$ which arises naturally from the regularised theta integral. In particular, we need to make a careful study of the properties of the section $F^{n, r}(s, \phi)$ and its restriction $f^{n, r}(s, \phi)$ to $G\left(W_{n}\right)$, such as the behavior of $f^{n, r}(s, \phi)$ when restricted to the subgroup $G\left(W_{n-1}\right)$, and the effect of the standard intertwining operator on $f^{n, r}(s, \phi)$. These key technical results are contained in Proposition 4.4. Proposition 9.8 and Lemma 9.12. When $r=n$, the map $F^{n, r}(s,-)$ was considered and studied in Q, Prop. 2.4].

As we noted, the second term identity leads to the following Rallis inner product formula for the theta lifting from $G\left(U_{n}\right)$ to $H\left(V_{r}\right)$. Since the Rallis inner product formula has been established in the convergent range and the first term range, we shall focus on the case

$$
\left(n+\epsilon_{0}\right)<m \leq 2 \cdot\left(n+\epsilon_{0}\right) .
$$

Then we have:

Theorem 1.3 (Rallis inner product formula). Suppose that

$$
\left(n+\epsilon_{0}\right)<m \leq 2 \cdot\left(n+\epsilon_{0}\right) \text { and } r \leq n
$$

so that we are either in the second term range or the convergent range, depending on whether $m \leq$ $\left(n+\epsilon_{0}\right)+r$ or not. Let $\pi$ be a cuspidal representation of $G\left(U_{n}\right)$ and consider its global theta lift $\Theta_{n, r}(\pi)$ to $H\left(V_{r}\right)$.

(i) Assume that $\Theta_{n, j}(\pi)=0$ for $j<r$, so that $\Theta_{n, r}(\pi)$ is cuspidal. Then for $\phi_{1}, \phi_{2} \in \omega_{\psi, U_{n}, V_{r}}$ and $f_{1}, f_{2} \in \pi$,

$$
\left\langle\theta\left(\phi_{1}, f_{1}\right), \theta\left(\phi_{2}, f_{2}\right)\right\rangle=[E: F] \cdot \operatorname{Val}_{s=s_{m, n}}\left(L\left(s+\frac{1}{2}, \pi\right) \cdot Z^{*}\left(s, \phi_{1} \otimes \overline{\phi_{2}}, f_{1}, f_{2}\right)\right),
$$


where

$$
s_{m, n}=\frac{m-n-\epsilon_{0}}{2}>0,
$$

$L(s, \pi)$ is the standard L-function of $\pi$, and $Z^{*}(s,-)$ denotes the normalized doubling zeta integral as in (11.3).

(ii) Assume further that for all places $v$ of $F$, the local theta lift $\Theta_{n, r}\left(\pi_{v}\right)$ is nonzero. Then $L\left(s+\frac{1}{2}, \pi\right)$ is holomorphic at $s=s_{m, n}$, so that in the context of (i),

$$
\left\langle\theta\left(\phi_{1}, f_{1}\right), \theta\left(\phi_{2}, f_{2}\right)\right\rangle=[E: F] \cdot L\left(s_{m, n}+\frac{1}{2}, \pi\right) \cdot Z^{*}\left(s_{m, n}, \phi_{1} \otimes \overline{\phi_{2}}, f_{1}, f_{2}\right) .
$$

As a consequence, we will be able to deduce the following local-global criterion (see Theorem 11.7) for the nonvanishing of global theta lifts.

Theorem 1.4 (Local-Global nonvanishing criterion). Assume the same conditions on $(m, n)$ as in Theorem 1.3. Let $\pi$ be a cuspidal representation of $G\left(U_{n}\right)$ and consider its global theta lift $\Theta_{n, r}(\pi)$ to $H\left(V_{r}\right)$. Assume that $\Theta_{n, j}(\pi)=0$ for $j<r$, so that $\Theta_{n, r}(\pi)$ is cuspidal.

(i) If $\Theta_{n, r}(\pi)$ is nonzero, then

(a) for all places $v, \Theta_{n, r}\left(\pi_{v}\right) \neq 0$, and

(b) $L\left(s_{m, n}+\frac{1}{2}, \pi\right) \neq 0$ i.e. nonzero holomorphic.

(ii) The converse to (i) holds when one assumes one of the following conditions:

- $\epsilon_{0}=-1$

- $\epsilon_{0}=0$ and $E_{v}=F_{v} \times F_{v}$ for all archimedean places $v$ of $F$;

- $\epsilon_{0}=1$ and $F$ is totally complex;

- $m=d(n)+1$.

(iii) In general, under the conditions (a) and (b) in (i), there is an $\epsilon$-Hermitian space $V^{\prime}$ over $E$ such that

- $V^{\prime} \otimes F_{v} \cong V \otimes F_{v}$ for every finite or complex place of $F$;

- the global theta lift $\Theta_{U_{n}, V^{\prime}}(\pi)$ of $\pi$ to $H\left(V^{\prime}\right)$ is nonzero.

The reason for not having the full converse to (i) is because of certain local issues at real places of $F$ which still need to be settled (see Proposition [11.6). To be more specific, one needs to show that the nonvanishing of the local theta lift $\Theta_{n, r}\left(\pi_{v}\right)$ is equivalent to the nonvanishing of the local normalized doubling zeta integral associated to $\pi_{v}$ on a certain submodule of the degenerate principal series representation. Because the structure of this degenerate principal series representation on $G\left(W_{n}\right)(\mathbb{R})$ is more complicated in the cases not covered in (ii), we could not show the desired equivalence. Once this archimedean issue can be settled, the converse to (i) will hold in general.

We note in closing that for the purpose of the local-global nonvanishing criterion, it is sufficient to show that the inner product of the theta lifts is proportional to the $L$-value, without having to be precise about the constant of proportionality. However, a precise Rallis inner product formula (with every constant determined) is useful for other purposes. For example, it was used in the proof of cases of the refined Gross-Prasad conjecture in [GI], [Ha and Q . In addition, it was used crucially in the work of Harris on period relations, and the work of Harris-Li-Skinner [HLS] on $p$-adic L-functions.

Acknowledgments: The debt that this paper owes to the fundamental work of Kudla and Rallis should be evident to the reader. We thank Atsushi Ichino for his help in the proof of Lemma 3.4(ii), Shunsuke 
Yamana for sending us his preprint [Y4 and for his helpful comments on this manuscript, Soo Teck Lee for his help in the proof of Proposition 6.4 and Chengbo Zhu for helpful discussion concerning the preprint [LZ4].

The second author thanks the National University of Singapore for hosting his visit in Spring 2012 while he and the first author worked on the paper. The writing of the paper was completed when the first and third authors visited the IHES in July 2012; they thank the IHES for its support and for providing a peaceful yet stimulating working environment. The first author is partially supported by a grant from the National University of Singapore. The third author is partially supported by NSF grant DMS-1215419.

Finally, we thank the referee of our paper for the many useful comments and suggestions, and for pointing out various inaccuracies in a first draft of this paper, especially with regards to some boundary cases of our main result which require some special considerations.

\section{Notation and Preliminaries}

In this section, we fix some notation and introduce the objects which intervene in this paper.

2.1. Fields. Let $F$ be a number field with ring of adeles $\mathbb{A}$. Fix a non-trivial additive character $\psi=\otimes_{v}^{\prime} \psi_{v}$ on $F \backslash \mathbb{A}$. We let $E$ be either $F$ or a quadratic extension of $F$. We shall regard $\psi$ as a character of $\mathbb{A}_{E}$ by composition with the trace map $\operatorname{Tr}_{E / F}$. Moreover, let $\chi_{E}$ denote the (possibly trivial) quadratic character of $\mathbb{A}^{\times}$associated to $E / F$ by global class field theory. By $|-|$, we always mean the absolute value on $\mathbb{A}_{E}^{\times}$rather than on $\mathbb{A}^{\times}$. Occasionally, we use the notation $|-|$for the absolute value of a local field, but this should be clear from the context.

For a vector space $X$ over $E$, by $\mathcal{S}(X)(\mathbb{A})$ we mean the space of Schwartz-Bruhat functions on $X \otimes \mathbb{A}$ and so $\mathcal{S}(X)(\mathbb{A})=\otimes_{v}^{\prime} \mathcal{S}(X)\left(F_{v}\right)$, where $\mathcal{S}(X)\left(F_{v}\right)$ is the space of Schwartz-Bruhat functions on $X \otimes F_{v}$. We sometimes omit $(\mathbb{A})$ or $\left(F_{v}\right)$ and simply write $\mathcal{S}(X)$ when there is no danger of confusion.

2.2. $\epsilon$-Hermitian Spaces. Throughout the paper, we shall fix the sign

$$
\epsilon= \pm 1
$$

Let $V_{0}$ be an $m_{0}$-dimensional vector space over $E$ equipped with a nondegenerate anisotropic $\epsilon$-Hermitian form $(-,-)$. With $\mathbb{H}$ denoting the hyperbolic plane, i.e. the split $\epsilon$-Hermitian space of dimension 2 , we set

$$
V_{r}=V_{0} \oplus \mathbb{H}^{r}
$$

and let

$$
m=\operatorname{dim}_{E} V=m_{0}+2 r .
$$

The family of spaces $\left\{V_{r}: r \geq 0\right\}$ forms a Witt tower of $\epsilon$-Hermitian spaces.

We now make two basic definitions. Set

$$
\epsilon_{0}=\left\{\begin{array}{l}
\epsilon, \text { if } E=F \\
0, \text { if } E \neq F
\end{array}\right.
$$

and

$$
d(n)=n+\epsilon_{0}
$$

for $n \in \mathbb{Z}$. 
Let $H_{r}=H\left(V_{r}\right)$ be the associated isometry group, so that

$$
H_{r} \cong \begin{cases}\mathrm{O}_{m}, & \text { if } \epsilon_{0}=1 \\ \mathrm{Sp}_{m}, & \text { if } \epsilon_{0}=-1 \\ \mathrm{U}_{m}, & \text { if } \epsilon_{0}=0\end{cases}
$$

The family of groups $\left\{H_{r}: r \geq 0\right\}$ forms a Witt tower of classical groups.

The space $V_{r}$ has a maximal isotropic space $X_{r}$ of dimension $r$, so that

$$
V_{r}=X_{r} \oplus V_{0} \oplus X_{r}^{*}
$$

Fix an ordered basis $\left\{x_{1}, \ldots, x_{r}\right\}$ of $X_{r}$, with corresponding dual basis $\left\{x_{r}^{*}, \cdots, x_{1}^{*}\right\}$ for $X_{r}^{*}$, so that

$$
X_{r}=E \cdot x_{1} \oplus \cdots \oplus E \cdot x_{r} \quad \text { and } \quad X_{r}^{*}=E \cdot x_{r}^{*} \oplus \cdots \oplus E \cdot x_{1}^{*}
$$

with

$$
\left(x_{i}, x_{j}\right)=\left(x_{i}^{*}, x_{j}^{*}\right)=0 \quad \text { and } \quad\left(x_{i}, x_{j}^{*}\right)=\delta_{i j} .
$$

Let

$$
P\left(X_{r}\right)=M\left(X_{r}\right) \cdot N\left(X_{r}\right)
$$

be the maximal parabolic subgroup of $H_{r}$ which stabilizes the space $X_{r}$. Then its Levi factor is

$$
M\left(X_{r}\right) \cong \mathrm{GL}\left(X_{r}\right) \times H\left(V_{0}\right) .
$$

To simplify notation, we shall sometimes write $P_{r}$ in place of $P\left(X_{r}\right)$. Note that in all cases, we have

$$
\pi_{0}\left(H_{r}\right)=\pi_{0}\left(H_{0}\right) \quad \text { if } r>0,
$$

except when $\epsilon_{0}=1$ and $m_{0}=0$, in which case $H_{r}=\mathrm{O}_{r, r}$ and $H_{0}$ is trivial so that

$$
\# \pi_{0}\left(H_{r} / H_{0}\right)=2 \text {. }
$$

Here $\pi_{0}$ indicates the set of connected components.

The group $H_{r}$ comes equipped with a family of maximal compact subgroups $\left\{K_{H_{r, v}}\right\}$, such that $K_{H_{r, v}}$ is hyperspecial for almost all places $v$ of $F$. We may and do assume that $K_{H_{r, v}}$ is a special maximal compact subgroup for all finite $v$. Then

$$
K_{H_{r}}=\prod_{v} K_{H_{r, v}} \subset H_{r}(\mathbb{A})
$$

is a maximal compact subgroup of $H_{r}(\mathbb{A})$ and one has the Iwasawa decomposition

$$
H_{r}(\mathbb{A})=P\left(X_{r}\right)(\mathbb{A}) \cdot K_{H_{r}} .
$$

We may also ensure that $K_{H_{r}} \cap \mathrm{GL}\left(X_{r}\right)(\mathbb{A})$ is a maximal compact subgroup of $\mathrm{GL}\left(X_{r}\right)(\mathbb{A})$.

2.3. $-\epsilon$-Hermitian Spaces. Similarly, let $W_{n}$ be a $2 n$-dimensional vector space over $E$ equipped with a nondegenerate $-\epsilon$-Hermitian form $\langle-,-\rangle$ and a maximal isotropic subspace $Y_{n}$ of dimension $n$, so that

$$
W_{n}=Y_{n} \oplus Y_{n}^{*}
$$

Let $G=G_{n}=G\left(W_{n}\right)$ be the associated isometry group or the unique two-fold cover thereof, according to

$$
G_{n}= \begin{cases}\mathrm{Sp}_{2 n}, & \text { if } \epsilon_{0}=1 \text { and } m_{0} \text { is even; } \\ \mathrm{Mp}_{2 n}, & \text { if } \epsilon_{0}=1 \text { and } m_{0} \text { is odd; } \\ \mathrm{O}_{2 n}, & \text { if } \epsilon_{0}=-1 \\ \mathrm{U}_{2 n}, & \text { if } \epsilon_{0}=0\end{cases}
$$

Thus, in addition to the space $W_{n}$, the group $G_{n}$ depends on the space $V_{0}$ (or rather the parity of its dimension) in the first two cases. 
We fix an ordered basis $\left\{y_{1}, \ldots, y_{n}\right\}$ of $Y_{n}$ and corresponding dual basis $\left\{y_{n}^{*}, \ldots, y_{1}^{*}\right\}$ for $Y_{n}^{*}$, so that

$$
Y_{n}=E \cdot y_{1} \oplus \cdots \oplus E \cdot y_{n} \quad \text { and } \quad Y_{n}^{*}=E \cdot y_{n}^{*} \oplus \cdots \oplus E \cdot y_{1}^{*} .
$$

For any subspace $Y_{r}:=\left\langle y_{1}, \ldots, y_{r}\right\rangle \subset Y_{n}$, let

$$
Q\left(Y_{r}\right)=L\left(Y_{r}\right) \cdot U\left(Y_{r}\right)
$$

denote the maximal parabolic subgroup fixing $Y_{r}$. Then its Levi factor is

$$
L\left(Y_{r}\right) \cong \mathrm{GL}\left(Y_{r}\right) \times G_{n-r} .
$$

As before, we shall sometimes write $Q_{r}$ in place of $Q\left(Y_{r}\right)$. Moreover, if there is a need to indicate that $Q_{r}$ is a subgroup of $G_{n}$, we shall write $Q_{r}^{n}$. When $r=n, Q\left(Y_{r}\right)$ is a Siegel parabolic subgroup of $G_{n}$.

The unipotent radical $U\left(Y_{r}\right)$ of $Q\left(Y_{r}\right)$ sits in a short exact sequence

$$
1 \longrightarrow Z\left(Y_{r}\right) \longrightarrow N\left(Y_{r}\right) \longrightarrow V_{n-r} \otimes Y_{r} \longrightarrow 1
$$

where

$$
Z\left(Y_{r}\right)=\left\{\epsilon \text {-Hermitian forms on } Y_{r}^{*}\right\} \subset \operatorname{Hom}_{F}\left(Y_{r}^{*}, Y_{r}\right) .
$$

Thus, when $r=n, N\left(Y_{n}\right)=Z\left(Y_{n}\right)$ is abelian.

The following table summarizes the groups discussed so far:

\begin{tabular}{|c|c|c|c|}
\hline \multicolumn{2}{|c|}{} & $G_{n}=G\left(W_{n}\right)$ & $H_{r}=H\left(V_{r}\right)$ \\
\hline \multirow{2}{*}{$\epsilon_{0}=1$} & $m_{0}$ even & $\mathrm{Sp}_{2 n}$ & $\mathrm{O}_{m}$ \\
\cline { 2 - 4 } & $m_{0}$ odd & $\mathrm{Mp}_{2 n}$ & $\mathrm{O}_{m}$ \\
\hline \multicolumn{2}{|c|}{$\epsilon_{0}=-1$} & $\mathrm{O}_{2 n}$ & $\mathrm{Sp}_{m}$ \\
\hline \multicolumn{2}{|c|}{$\epsilon_{0}=0$} & $\mathrm{U}_{2 n}$ & $\mathrm{U}_{m}$ \\
\hline \hline \multicolumn{2}{|c|}{ Parabolic } & $Q_{r}=Q\left(Y_{r}\right)=L\left(Y_{r}\right) \cdot U\left(Y_{r}\right)$ & $P_{r}=P\left(X_{r}\right)=M\left(X_{r}\right) \cdot N\left(X_{r}\right)$ \\
\hline \multicolumn{2}{|c|}{ Levi factor } & $L\left(Y_{r}\right)=\mathrm{GL}\left(Y_{r}\right) \times G_{n-r}$ & $M\left(X_{r}\right)=\mathrm{GL}\left(X_{r}\right) \times H\left(V_{0}\right)$ \\
\hline
\end{tabular}

2.4. Metaplectic case. The case when $G_{n}(\mathbb{A})$ is the metaplectic group $\operatorname{Mp}_{2 n}(\mathbb{A})$ deserves further comments. In this case, a parabolic subgroup of $G_{n}(\mathbb{A})$ is simply the inverse image of a parabolic subgroup of $\mathrm{Sp}_{2 n}(\mathbb{A})$. We will follow the notation and conventions in $[\mathrm{GS}]$ for the structural issues regarding $\mathrm{Mp}_{2 n}(\mathbb{A})$. For example, the Levi subgroup of a parabolic $Q\left(Y_{r}\right)(\mathbb{A})$ is:

$$
L\left(Y_{r}\right)(\mathbb{A})=\widetilde{G L}_{r}(\mathbb{A}) \times_{\mu_{2}} \operatorname{Mp}_{2 n-2 r}(\mathbb{A})
$$

where $\widetilde{G L}_{r}(\mathbb{A})$ is the two-fold cover of $\mathrm{GL}_{r}(\mathbb{A})$ defined by the Hilbert symbol, namely

$$
\widetilde{\mathrm{GL}}_{r}(\mathbb{A})=\mathrm{GL}_{r}(\mathbb{A}) \times\{ \pm 1\}
$$

as a set and the group structure is given by

$$
\left(g_{1}, \eta_{1}\right) \cdot\left(g_{2}, \eta_{2}\right)=\left(g_{1} g_{2}, \eta_{1} \eta_{2}\left(\operatorname{det} g_{1}, \operatorname{det} g_{2}\right)_{\mathbb{A}}\right) \quad \text { for } g_{i} \in \mathrm{GL}_{r}(\mathbb{A}) \text { and } \eta_{i} \in\{ \pm 1\},
$$

where $(-,-)_{\mathbb{A}}$ is the Hilbert symbol. The determinant map of $\mathrm{GL}_{r}(\mathbb{A})$ lifts to a homomorphism

$$
\operatorname{det}: \widetilde{\mathrm{GL}}_{r}(\mathbb{A}) \longrightarrow \widetilde{\mathrm{GL}}_{1}(\mathbb{A}) \text {. }
$$

By the theory of Weil indices, the additive character $\psi$ gives rise to a genuine character

$$
\chi_{\psi}: \widetilde{\mathrm{GL}}_{1}(\mathbb{A}) \longrightarrow \mathbb{C}^{\times}
$$

Composing with det, one obtains a genuine character $\chi_{\psi}$ odet of $\widetilde{\mathrm{GL}}_{r}(\mathbb{A})$. The map $\tau \mapsto \tilde{\tau}_{\psi}=\left(\chi_{\psi} \circ \operatorname{det}\right) \otimes \tau$ then gives an identification (depending on $\psi$ ) of the irreducible representations of $\mathrm{GL}_{r}(\mathbb{A})$ with the irreducible genuine representations of $\widetilde{\mathrm{GL}}_{r}(\mathbb{A})$. 
In order to unify and simplify notation, we shall henceforth write $\tau$ for a genuine representation of $L\left(Y_{r}\right)(\mathbb{A})$ in the metaplectic case, when we actually mean the genuine representation $\tilde{\tau}_{\psi}$. Also for a parabolic subgroup $Q\left(Y_{r}\right)(\mathbb{A})$ of $\mathrm{Mp}_{2 n}(\mathbb{A})$ and a representation $\tau \otimes \sigma$ of $L\left(Y_{r}\right)(\mathbb{A})=\widetilde{\mathrm{GL}}\left(Y_{r}\right)(\mathbb{A}) \times_{\mu_{2}}$ $\operatorname{Mp}_{2 n-2 r}(\mathbb{A})$, one may consider the normalized induced representation $\operatorname{Ind}_{Q(Y)(\mathbb{A})}^{\operatorname{Mp}_{2 n}(\mathbb{A})}\left(\tau|\operatorname{det}|^{s} \otimes \sigma\right)$, keeping in mind that $\tau$ denotes the genuine representation $\tilde{\tau}_{\psi}$. In particular, this notion of induced representation depends on $\psi$, though we suppress $\psi$ from the notation.

2.5. Measures. Having fixed the additive character $\psi$ of $F \backslash \mathbb{A}$, we fix the Haar measure $d x_{v}$ on $F_{v}$ (for all $v$ ) to be self-dual with respect to $\psi_{v}$. The product measure $d x$ on $\mathbb{A}$ is independent of the choice of $\psi$ and is the Tamagawa measure of $\mathbb{A}$. For any algebraic group $G$ over $F$, we always use the Tamagawa measure on $G(\mathbb{A})$ when $G(\mathbb{A})$ is unimodular. This applies to the groups $G_{n}(\mathbb{A})$ and $H_{r}(\mathbb{A})$, as well as the Levi subgroups and unipotent radical of their parabolic subgroups. In addition, for any compact group $K$, we always use the Haar measure $d k$ with respect to which $K$ has volume 1 .

Finally, we need to fix a measure for the metaplectic group $\mathrm{Mp}_{2 n}(\mathbb{A})$. Recall that one has a short exact sequence

$$
1 \longrightarrow \mu_{2} \longrightarrow \mathrm{Mp}_{2 n}(\mathbb{A}) \stackrel{\pi}{\longrightarrow} \operatorname{Sp}_{2 n}(\mathbb{A}) \longrightarrow 1 \text {. }
$$

Having fixed the Tamagawa measure $d g$ on $\operatorname{Sp}_{2 n}(\mathbb{A})$, we fix the measure $d \tilde{g}$ on $\operatorname{Mp}_{2 n}(\mathbb{A})$ such that $\pi_{*}(d \tilde{g})=d g$, so that

$$
\int_{\mathrm{Sp}_{2 n}(F) \backslash \mathrm{Mp}_{2 n}(\mathbb{A})} d \tilde{g}=1 .
$$

We shall call this $d \tilde{g}$ the Tamagawa measure for $\operatorname{Mp}_{2 n}(\mathbb{A})$. We adopt the same convention for the Tamagawa measure on the nonlinear cover $\tilde{G L}_{r}(\mathbb{A})$ of $\mathrm{GL}_{r}(\mathbb{A})$ which intervenes in the parabolic subgroups of $\operatorname{Mp}_{2 n}(\mathbb{A})$.

In any case, we write $\tau(G)$ for the Tamagawa number of $G$. When the identity component $G^{0}$ has no $F$-rational characters, $\tau(G)$ is simply defined by:

$$
\tau(G)=\int_{[G]} d g
$$

and a more delicate definition is needed in the general case. In any case, we have

except when

$$
\tau\left(H_{r}\right)= \begin{cases}1, & \text { if } \epsilon_{0} \neq 0 \\ 2, & \text { if } \epsilon_{0}=0\end{cases}
$$

- $\epsilon_{0}=1, m_{0}=1$ and $r=0$, in which case $H_{r}=\mathrm{O}_{1}$ has $\tau\left(\mathrm{O}_{1}\right)=1 / 2$ (see We2]);

- $\epsilon_{0}=1, m_{0}=0$ and $r=1$, in which case $H_{r}=\mathrm{O}_{1,1}$ is associated to the split binary quadratic form and $H_{r}^{0} \cong \mathbb{G}_{m}$, so that $\tau\left(\mathrm{O}_{1,1}\right)=1 / 2$.

2.6. Complementary Spaces. With $W_{n}$ fixed, one may associate to each $V_{r}$ a complementary space $V_{r^{\prime}}$ in the same Witt tower, characterized by

$$
m_{0}+r+r^{\prime}=d(n) .
$$

Of course, this only makes sense if $r^{\prime} \geq 0$. Thus, the notion of complementary spaces is only relevant when

$$
0 \leq m_{0}+r \leq d(n)
$$

When $V_{r^{\prime}}$ exists, and $r \geq r^{\prime}$, we may write:

$$
V_{r}=X_{r-r^{\prime}}^{\prime} \oplus V_{r^{\prime}} \oplus X_{r-r^{\prime}}^{\prime *}
$$


where

We set

$$
X_{r-r^{\prime}}^{\prime}=\left\langle x_{r^{\prime}+1}, \ldots, x_{r}\right\rangle
$$

$$
m^{\prime}=\operatorname{dim} V_{r^{\prime}}=m_{0}+2 r^{\prime} .
$$

2.7. Ikeda's map. Suppose that $V_{r} \supset V_{r^{\prime}}$ (not necessarily complementary spaces). Then one may write

$$
V_{r}=X_{r-r^{\prime}}^{\prime} \oplus V_{r^{\prime}} \oplus X_{r-r^{\prime}}^{\prime *}
$$

We define a map

given by

$$
\mathrm{Ik}^{n, r, r^{\prime}}: \mathcal{S}\left(Y_{n}^{*} \otimes V_{r}\right)(\mathbb{A}) \longrightarrow \mathcal{S}\left(Y_{n}^{*} \otimes V_{r^{\prime}}\right)(\mathbb{A})
$$

$$
\mathrm{Ik}^{n, r, r^{\prime}}(\phi)(a)=\int_{\left(Y_{n}^{*} \otimes X_{r-r^{\prime}}^{\prime}\right)(\mathbb{A})} \phi(x, a, 0) d x,
$$

for $a \in\left(Y_{n}^{*} \otimes V_{r^{\prime}}\right)(\mathbb{A})$. Thus, $\mathrm{Ik}^{n, r, r^{\prime}}$ is the composite

$$
\begin{gathered}
\mathcal{S}\left(Y_{n}^{*} \otimes V_{r}\right)=\mathcal{S}\left(Y_{n}^{*} \otimes V_{r^{\prime}}\right) \otimes \mathcal{S}\left(Y_{n}^{*} \otimes\left(X_{r-r^{\prime}}^{\prime}+X_{r-r^{\prime}}^{\prime *}\right)\right) \\
\downarrow I d \otimes \mathcal{F}_{1} \\
\mathcal{S}\left(Y_{n}^{*} \otimes V_{r^{\prime}}\right) \otimes \\
\mathcal{S}\left(W_{n} \otimes X_{r-r^{\prime}}^{\prime}\right) \\
\downarrow I d \otimes e v_{0} \\
\mathcal{S}\left(Y_{n}^{*} \otimes V_{r^{\prime}}\right)
\end{gathered}
$$

where

$$
\mathcal{F}_{1}: \mathcal{S}\left(Y_{n}^{*} \otimes\left(X_{r-r^{\prime}}^{\prime}+X_{r-r^{\prime}}^{\prime *}\right)\right) \longrightarrow \mathcal{S}\left(W_{n} \otimes X_{r-r^{\prime}}^{\prime}\right)
$$

is the partial Fourier transform in the subspace $\left(Y_{n}^{*} \otimes X_{r-r^{\prime}}^{\prime *}(\mathbb{A})\right.$, and $e v_{0}$ is evaluation at 0 . It is clear that if $r^{\prime \prime}<r^{\prime}<r$, one has

$$
\mathrm{Ik}^{n, r^{\prime}, r^{\prime \prime}} \circ \mathrm{Ik}^{n, r, r^{\prime}}=\mathrm{Ik}^{n, r, r^{\prime \prime}} .
$$

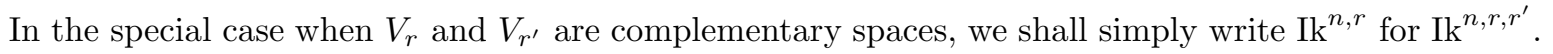
The map $\mathrm{Ik}^{n, r}$ was used by $\mathrm{T}$. Ikeda in his refinement ([Ik]) of the first term identity of Kudla-Rallis ([KR5]). Thus we call $\mathrm{Ik}^{n, r}$ (more generally $\mathrm{Ik}^{n, r, r^{\prime}}$ ) an Ikeda map.

2.8. Weil representation. We choose a Hecke character $\chi$ as follows:

$$
\chi=\chi_{V_{0}}=\left\{\begin{array}{l}
\text { the quadratic character of } \mathbb{A}^{\times} \text {associated to } \operatorname{disc}\left(V_{0}\right), \text { if } \epsilon_{0}=1 ; \\
\text { the trivial character of } \mathbb{A}^{\times}, \text {if } \epsilon_{0}=-1 ; \\
\text { a character of } \mathbb{A}_{E}^{\times} \text {such that }\left.\chi\right|_{\mathbb{A} \times}=\chi_{E}^{m_{0}}, \text { if } \epsilon_{0}=0 .
\end{array}\right.
$$

Note that when $\epsilon_{0}= \pm 1, \chi$ is completely determined by $V_{0}$.

Associated to the pair $(\psi, \chi)$, the group $G_{n}(\mathbb{A}) \times H_{r}(\mathbb{A})$ has a distinguished representation $\omega_{n, r}=$ $\omega_{\psi, \chi, W_{n}, V_{r}}$ known as the Weil representation. The Weil representation can be realized on the space of Schwartz-Bruhat functions $\mathcal{S}\left(Y_{n}^{*} \otimes V_{r}\right)(\mathbb{A})$. The action is given by the following formulas.

$$
\left\{\begin{array}{l}
\omega_{n, r}(1, h) \phi(x)=\phi\left(h^{-1} \cdot x\right) \text { if } h \in H_{r}(\mathbb{A}) ; \\
\omega_{n, r}(a, 1) \phi(x)=\chi(\operatorname{det}(a)) \cdot|\operatorname{det}(a)|^{m / 2} \cdot \phi\left(a^{-1} \cdot x\right), \text { for } a \in L\left(Y_{n}\right)(\mathbb{A})=\operatorname{GL}\left(Y_{n}\right)(\mathbb{A}) ; \\
\omega_{n, r}(u, 1) \phi(x)=\psi\left(\frac{1}{2} \cdot\langle u(x), x\rangle\right) \cdot \phi(x), \text { for } u \in N\left(Y_{n}\right)(\mathbb{A}) \subset \operatorname{Hom}_{F}\left(Y_{n}^{*}, Y_{n}\right)(\mathbb{A}), \\
\omega_{n, r}\left(w_{0}, 1\right) \phi(x)=\gamma_{V_{r}, W_{n}} \cdot \int_{V_{r} \otimes Y_{n}^{*}(\mathbb{A})} \phi(y) \cdot \psi(\langle x, y\rangle) d y,
\end{array}\right.
$$


where $w_{0} \in G_{n}(F)$ is the Weyl group element $y_{i} \mapsto-\epsilon \cdot y_{i}^{*}$ and $y_{i}^{*} \mapsto y_{i}$, and $\gamma_{V_{r}, W_{n}}$ is a certain root of unity which we will not explicate here.

Note that the Ikeda map

$$
\mathrm{Ik}^{n, r, r^{\prime}}: \mathcal{S}\left(V_{r} \otimes Y_{n}^{*}\right)(\mathbb{A}) \longrightarrow \mathcal{S}\left(V_{r^{\prime}} \otimes Y_{n}^{*}\right)(\mathbb{A})
$$

is $G_{n}(\mathbb{A}) \times H_{r^{\prime}}(\mathbb{A})$-equivariant.

2.9. The Fourier transform $\mathcal{F}_{n, r}$. On the other hand, one has the partial Fourier transform

$$
\mathcal{F}_{n, r}: \mathcal{S}\left(Y_{n}^{*} \otimes V_{r}\right)(\mathbb{A}) \longrightarrow \mathcal{S}\left(W_{n} \otimes X_{r}^{*}\right)(\mathbb{A}) \otimes \mathcal{S}\left(Y_{n}^{*} \otimes V_{0}\right)(\mathbb{A})
$$

which is given by integration over the subspace $\left(Y_{n}^{*} \otimes X_{r}\right)(\mathbb{A})$. We may regard $\mathcal{F}_{n, r}(\phi)$ as a function on $\left(W_{n} \otimes X_{r}^{*}\right)(\mathbb{A})$ taking values in $\mathcal{S}\left(Y_{n}^{*} \otimes V_{0}\right)(\mathbb{A})$. Transferring the action of $G_{n}(\mathbb{A}) \times H_{r}(\mathbb{A})$ via $\mathcal{F}_{n, r}$, we see for example that:

$$
\left\{\begin{array}{l}
\omega_{n, r}(g, 1) \mathcal{F}_{n, r}(\phi)(x)=\omega_{n, 0}(g)\left(\mathcal{F}_{n, r}(\phi)\left(g^{-1} \cdot x\right)\right) \text { for } g \in G_{n}(\mathbb{A}) \\
\omega_{n, r}(1, a) \mathcal{F}_{n, r}(\phi)(x)=|\operatorname{det}(a)|^{n} \cdot \mathcal{F}_{n, r}(\phi)(x \circ a), \text { for } a \in \operatorname{GL}\left(X_{r}\right)(\mathbb{A}) .
\end{array}\right.
$$

A bit of care is needed to interpret the representation $\omega_{n, 0}$ above when $m_{0}=0$. In that case, $\mathcal{S}\left(Y_{n}^{*} \otimes\right.$ $\left.V_{0}\right)(\mathbb{A})=\mathbb{C}$ is a 1-dimensional representation of $G_{n}$. When $\epsilon_{0}= \pm 1$ (and $m_{0}=0$ ), $\omega_{n, 0}$ is the trivial representation. When $\epsilon_{0}=0$, one has an isomorphism

$$
\iota: E^{\times} / F^{\times} \longrightarrow E_{1}
$$

where $E_{1}$ denotes the set of norm 1 elements in $E$, given by

$$
\iota(x)=x / x^{c} .
$$

Moreover, since $m_{0}=0, \chi$ is a character of $\mathbb{A}_{E}^{\times} / \mathbb{A}^{\times}$, so that one has a character $\chi \circ \iota^{-1} \circ \operatorname{det}_{G_{n}}$ of $G_{n}(\mathbb{A})$. Then one has

$$
\omega_{n, 0}=\chi \circ \iota^{-1} \circ \operatorname{det}_{G_{n}} .
$$

2.10. Automorphic forms. For an algebraic group $G$ over $F$, we set

$$
[G]:=G(F) \backslash G(\mathbb{A}) .
$$

We denote by $\mathcal{A}(G)$ the space of automorphic forms on $G$. The elements in $\mathcal{A}(G)$ are smooth automorphic forms on $[G]$ and we do not impose the usual $K$-finiteness condition. Hence the full group $G(\mathbb{A})$ acts on $\mathcal{A}(G)$.

2.11. Automorphic Theta distribution. The Weil representation $\omega_{n, r}$ has an automorphic realization

$$
\theta_{n, r}: \mathcal{S}\left(Y_{n}^{*} \otimes V_{r}\right)(\mathbb{A}) \longrightarrow \text { Functions on }\left[G_{n}\right] \times\left[H_{r}\right]
$$

given by

$$
\theta_{n, r}(\phi)(g, h)=\sum_{x \in\left(Y_{n}^{*} \otimes V_{r}\right)(F)}\left(\omega_{n, r}(g, h) \phi\right)(x) .
$$

\section{The Regularized Theta Integral}

In this section, we recall the definition of the theta integral and its regularization defined by KudlaRallis. 
3.1. Theta Integral. The theta integral we are interested in is:

$$
I_{n, r}(\phi)(g):=\frac{1}{\tau\left(H_{r}\right)} \cdot \int_{\left[H_{r}\right]} \theta_{n, r}(\phi)(g, h) d h .
$$

Note that the measure used in the above integral is effectively $\tau\left(H_{r}\right)^{-1} \cdot d h$, with respect to which $\left[H_{r}\right]$ has volume 1 (when $H_{r} \neq \mathrm{O}_{1,1}$ ). This agrees with the convention used in [KR1, KR2, IK, I1, I2, I3, IY2] when $H_{r} \neq \mathrm{O}_{1,1}$, but the convention is different in the $\mathrm{O}_{1,1}$ case.

The theta integral $I_{n, r}(\phi)$ is an automorphic form on $G_{n}$ if the integral converges absolutely. By Weil We1, it is known that the above integral converges if and only if

$$
r=0 \quad \text { or } \quad m-r=m_{0}+r>d(n) .
$$

We call this the Weil's convergent range.

In particular, the pair $\left(W_{n}, V_{0}\right)$ is in this range. Then for $\phi_{0} \in \mathcal{S}\left(Y_{n}^{*} \otimes V_{0}\right)(\mathbb{A})$, we have the theta integral $I_{n, 0}\left(\phi_{0}\right) \in \mathcal{A}\left(G_{n}\right)$. We set

$$
\Theta_{n, 0}\left(V_{0}\right)=\left\{I_{n, 0}\left(\phi_{0}\right): \phi_{0} \in \mathcal{S}\left(Y_{n}^{*} \otimes V_{0}\right)(\mathbb{A})\right\} \subset \mathcal{A}\left(G_{n}\right)
$$

which is an automorphic (sub)representation of $G_{n}$. If $V_{0}=0$, then $\Theta_{n, 0}\left(V_{0}\right)$ is interpreted to be the trivial representation of $G_{n}$ if $\epsilon_{0}= \pm 1$, and is interpreted to be the character $\chi \circ \iota^{-1} \circ \operatorname{det}_{G_{n}}$ as in (2.2) if $\epsilon_{0}=0$.

The classical Siegel-Weil formula, first formulated in We1 and extended by Kudla-Rallis in [KR1, determines the theta integral $I_{n, 0}\left(\phi_{0}\right)$ as an Eisenstein series on $G_{n}$.

3.2. Regularization. In [KR5, Kudla and Rallis defined a regularization of the integral $I_{n, r}(\phi)$ beyond the Weil's convergent range. More precisely, we suppose that

$$
r>0, \text { and } m-r \leq d(n) .
$$

Note that the second inequality implies that

$$
r \leq d(n) \leq n+1 .
$$

We further impose (as did Kudla-Rallis) that

$$
r \leq n,
$$

or equivalently that the $F$-rank of $G_{n}$ is at least that of $H_{r}$. Note that this is only an extra condition when $\epsilon_{0}=1$; when $\epsilon_{0}=-1$ or 0 , it follows automatically from (3.1) since $d(n) \leq n$. Indeed, the only case satisfying (3.1) but not (3.2) is when $\epsilon_{0}=1$ and $m_{0}=0$ so that $H_{r}=\mathrm{O}_{n+1, n+1}$ (split) and $G_{n}=\mathrm{Sp}_{2 n}$. We also note that under (3.1):

$$
0<m \leq 2 \cdot d(n)
$$

For a given place $v$, one can find an element $z_{G}$ (resp. $z_{H}$ ) in the center of universal enveloping algebra of $\mathfrak{g}_{v}$ (resp. $\mathfrak{h}_{v}$ ) for $v$ real (KR5) or in the spherical Hecke algebra of $G_{n}\left(F_{v}\right)$ (resp. $H_{r}\left(F_{v}\right)$ ) for $v$ non-archimedean ([1, I2, T1] ) so that

$$
\begin{aligned}
\omega_{n, r}\left(z_{G}\right) & =\omega_{n, r}\left(z_{H}\right), \\
\omega_{n, r}(g, h) \omega_{n, r}\left(z_{G}\right) & =\omega_{n, r}\left(z_{G}\right) \omega_{n, r}(g, h)
\end{aligned}
$$

(i.e. the action of $z_{G}$ (and hence $z_{H}$ ) commutes with the action of $G_{n}(\mathbb{A}) \times H_{r}(\mathbb{A})$ ), and such that the function $\theta_{n, r}\left(\omega_{n, r}\left(z_{G}\right) \varphi\right)(g,-)$ is rapidly decreasing on a Siegel domain of $H_{r}(\mathbb{A})$. It follows that one can integrate $\theta_{n, r}\left(\omega_{n, r}\left(z_{G}\right) \phi\right)$ against any automorphic form on $H_{r}$; we shall integrate it against an auxiliary Eisenstein series on $H_{r}$ to be defined next. 
3.3. Auxiliary Eisenstein series on $H_{r}$. Recall $P\left(X_{r}\right)$ is the parabolic subgroup of $H_{r}$ whose Levi is $M\left(X_{r}\right)=\mathrm{GL}\left(X_{r}\right) \times H\left(V_{0}\right)$. Consider the family of (normalized) induced representation

$$
I_{H_{r}}(s):=\operatorname{Ind}_{P\left(X_{r}\right)(\mathbb{A})}^{H_{r}(\mathbb{A})}|\operatorname{det}|^{s} \otimes \mathbf{1}_{H_{0}}
$$

where $|\operatorname{det}|^{s}$ is a character of $\operatorname{GL}\left(X_{r}\right)(\mathbb{A})$ and $\mathbf{1}_{H_{0}}$ is the trivial representation of $H_{0}(\mathbb{A})$. Let $f_{s}^{0} \in I_{H_{r}}(s)$ be the $K_{H_{r}}$-spherical standard section with $f_{s}^{0}(1)=1$. Then we define the Eisenstein series $E_{H_{r}}(s,-)$ on $H_{r}$ by

$$
E_{H_{r}}(s, h)=\sum_{\gamma \in P\left(X_{r}\right)(F) \backslash H_{r}(F)} f_{s}^{0}(\gamma h)
$$

for $h \in H_{r}(\mathbb{A})$ and $\operatorname{Re}(s) \gg 0$. We call $E_{H_{r}}(s,-)$ the auxiliary Eisenstein series.

We are interested in the point

$$
s=\rho_{H_{r}}:=\frac{m-r-\epsilon_{0}}{2} .
$$

We have:

$$
\operatorname{ord}_{s=\rho_{H_{r}}} E_{H_{r}}(s,-)=\left\{\begin{array}{l}
0, \text { if } H_{r}=\mathrm{O}_{1,1}, \text { i.e. } m=2, r=1 \text { and } \epsilon_{0}=1 ; \\
-1, \text { otherwise. }
\end{array}\right.
$$

The leading Laurent coefficient of $E_{H_{r}}(s,-)$ at $s=\rho_{H_{r}}$ is a constant function and we set:

$$
\kappa_{r}=\left\{\begin{array}{l}
E_{H_{r}}\left(\rho_{H_{r}}, h\right), \text { if } H_{r}=\mathrm{O}_{1,1} ; \\
\operatorname{Res}_{s=\rho_{H_{r}}} E_{H_{r}}(s, h), \text { otherwise. }
\end{array}\right.
$$

Note that when $H_{r}=\mathrm{O}_{1,1}$ (so that $r=1$ ), one has $P\left(X_{r}\right)=H_{r}^{0} \cong \mathbb{G}_{m}, \rho_{H_{r}}=0$ and $\kappa_{r}=2$. For a detailed discussion of this degenerate case, the reader can consult [KRS, §3.1, Pg. 499-503].

Now the regularizing element $z_{H}$ acts on $E_{H_{r}}(s,-)$ by a scalar $P_{n, r}(s)$ :

$$
z_{H} * E_{H_{r}}(s,-)=P_{n, r}(s) \cdot E_{H_{r}}(s,-) \text {. }
$$

Here the scalar $P_{n, r}(s)$ depends on the choice of $z_{H}$, though we suppress the dependence from our notation. The function $P_{n, r}(s)$ can be explicitly computed; see [KR5, Lemma 5.5.3], I1, p. 208], I2, p. 249] and [T1, Cor. 2.2.5] for the various cases.

There is a standard intertwining operator

$$
M_{H_{r}}(s): I_{H_{r}}(s) \longrightarrow I_{H_{r}}(-s)
$$

defined for $\operatorname{Re}(s) \gg 0$ by

$$
M_{H_{r}}(s) f_{s}(h)=\int_{N\left(X_{r}\right)(\mathbb{A})} f_{s}\left(w_{H_{r}} u h\right) d u
$$

for $f_{s} \in I_{H_{r}}(s)$, where $w_{H_{r}}$ is the element in $H_{r}(F)$ defined by

$$
w_{H_{r}}:\left\{\begin{array}{l}
x_{i} \mapsto \epsilon \cdot x_{i}^{*} \text { for } 1 \leq i \leq r \\
x_{i}^{*} \mapsto x_{i} \text { for } 1 \leq i \leq r ; \\
v_{0} \mapsto v_{0} \text { for } v_{0} \in V_{0}
\end{array}\right.
$$

For almost all $s, M_{H_{r}}(s)$ is an $H_{r}(\mathbb{A})$-equivariant isomorphism. In particular, there is a meromorphic scalar-valued function $c_{r}(s)$ such that

$$
M_{H_{r}}(s) f_{s}^{0}=c_{r}(s) \cdot f_{-s}^{0} .
$$

Together with the functional equation of Eisenstein series, one has:

$$
E_{H_{r}}(s,-)=c_{r}(s) \cdot E_{H_{r}}(-s,-) .
$$


The function $c_{r}(s)$ can be explicitly computed (see for example [Ik, $\S 9$ ] and [12, $\left.\S 9\right]$ ), but we shall not need its explicit value in this paper. The only information we need to know about the function $c_{r}(s)$ is the following innocuous looking but really quite important lemma:

Lemma 3.4. (i) At the point $s=\rho_{H_{r}}$,

$$
\operatorname{ord}_{s=\rho_{H_{r}}} c_{r}(s)=\operatorname{ord}_{s=\rho_{H_{r}}} E_{H_{r}}(s,-),
$$

and

$$
\operatorname{Val}_{s=\rho_{H_{r}}}\left(\frac{E_{H_{r}}(s,-)}{c_{r}(s)}\right)=\# \pi_{0}\left(H_{r} / H_{0}\right)= \begin{cases}1 & \text { if } H_{r} \neq \mathrm{O}_{r, r} \\ 2 & \text { if } H_{r}=\mathrm{O}_{r, r}\end{cases}
$$

In particular,

$$
\operatorname{ord}_{s=\rho_{H_{r}}} c_{r}(s) / c_{r-1}(s-1 / 2)=\left\{\begin{array}{l}
-1, \text { if } H_{r}=\mathrm{O}_{2,2} \text { or if } r=1 \text { and } H_{r} \neq \mathrm{O}_{1,1} \\
0, \text { otherwise. }
\end{array}\right.
$$

and its leading Laurent coefficient there is $\kappa_{r} / \kappa_{r-1} \cdot \# \pi_{0}\left(H_{r-1} / H_{r}\right)$. Here, we have set $c_{0}(s)=1$ and $\kappa_{0}=1$ by convention.

(ii) With respect to the Iwasawa decomposition $H_{r}(\mathbb{A})=M\left(X_{r}\right)(\mathbb{A}) \cdot N\left(X_{r}\right)(\mathbb{A}) \cdot K_{H_{r}}$, one has:

$$
\frac{1}{\tau\left(H_{r}\right)} \cdot \int_{H_{r}(\mathbb{A})} \varphi(h) d h=\kappa_{r} \cdot \frac{1}{\tau\left(H_{0}\right)} \cdot \int_{M\left(X_{r}\right)(\mathbb{A})} \int_{N\left(X_{r}\right)(\mathbb{A})} \int_{K_{H_{r}}} \varphi(m \cdot n \cdot k) d m d n d k,
$$

for any $\varphi \in C_{c}^{\infty}\left(H_{r}(\mathbb{A})\right)$, where $d h, d m$ and $d n$ are the Tamagawa measures on $H_{r}(\mathbb{A}), M\left(X_{r}\right)(\mathbb{A})$ and $N\left(X_{r}\right)(\mathbb{A})$, respectively and $d k$ is such that the volume of $K_{H_{r}}$ is 1 .

Proof. (i) We give the formal proof of (i) in 8 8.4, after we introduce the relevant notation for constant terms of Eisenstein series. But let us mention here that, by the theory of Eisenstein series, the analytic behavior of $E_{H_{r}}(s,-)$ is the same as that of its constant term along $N\left(X_{r}\right)$. Then (i) is saying that the analytic behavior of this constant term at $s=\rho_{H_{r}}$ is the same as that of the intertwining operator $M_{H_{r}}(s)$.

(ii) This follows from [I2, Lemma 9.1] and the computations in [Ik, §9] and [I2, §9], especially [Ik Thm. 9.6 and 9.7] and [12, Thm. 9.5 and 9.6]. We give a sketch of the proof.

Since the case when $H_{r}=\mathrm{O}_{1,1}$ can be easily verified by hand, we assume that $H_{r} \neq \mathrm{O}_{1,1}$ henceforth. According to [Ik, Lemma 9.1] and [I2, Lemma 9.1], if we define the constant $\alpha$ by

$$
d h=\alpha \cdot d m d n d k,
$$

then

$$
\alpha=\operatorname{Res}_{s=1} \frac{L\left(s, M_{r}\right)}{L\left(s, H_{r}\right)} \cdot\left(\prod_{v} \frac{L_{v}\left(1, H_{r}\right)}{L_{v}\left(1, M_{v}\right)} \cdot c_{r, v}\left(\rho_{H_{r}}\right)\right),
$$

where $L\left(s, H_{r}\right)$ is the Artin L-function associated to the Galois module $\operatorname{Hom}\left(H_{r}, \mathbb{G}_{m}\right) \otimes_{\mathbb{Z}} \mathbb{Q}$ with local component $L_{v}\left(s, H_{r}\right)$ at $v$, and analogously for $L\left(s, M_{r}\right)$. Similarly, $c_{r, v}(s)$ is the local component of $c_{r}(s)$ so that $M_{H_{r}, v}(s) f_{s, v}^{0}=c_{r, v}(s) \cdot f_{-s, v}^{0}$. Note that the ratio $L_{v}\left(1, H_{r}\right) / L_{v}\left(1, M_{r}\right)$ is equal to $1 / \zeta_{E_{v}}(1)$ or $1 / \zeta_{F_{v}}(1)$ in the various cases.

Now the value of $c_{r, v}(s)$ has been computed in [Ik, §9], [I1] and [12, §9], and is essentially a ratio of products of local Hecke L-functions. When one evaluates $c_{r, v}(s)$ at $s=\rho_{H_{r}}$, one obtains local Hecke Lvalues at $s>1$ except for a term $\beta_{v}(s)$ in the numerator which satisfies $\beta_{v}\left(\rho_{H_{r}}\right)=\zeta_{E_{v}}(1)$ or $\zeta_{F_{v}}(1)$. This term is cancelled by $L_{v}\left(1, H_{r}\right) / L_{v}\left(1, M_{r}\right)$, so that the Euler product on the RHS of (3.5) is absolutely convergent. 
Now the RHS of (3.5) looks very much like $\operatorname{Res}_{s=\rho_{H_{r}}} c_{r}(s)$. Indeed,

$$
\operatorname{Res}_{s=\rho_{H_{r}}} c_{r}(s)=\operatorname{Res}_{s=\rho_{H_{r}}} \beta(s) \cdot\left(\prod_{v} \frac{L_{v}(1, G)}{L_{v}\left(1, M_{v}\right)} \cdot c_{r, v}\left(\rho_{H_{r}}\right)\right),
$$

where $\beta(s)$ is the global zeta function with local component $\beta_{v}(s)$. Hence, we see that

$$
\alpha=\operatorname{Res}_{s=\rho_{H_{r}}} c_{r}(s) \cdot \operatorname{Res}_{s=1}\left(\frac{L\left(s, M_{r}\right)}{L\left(s, H_{r}\right)}\right) / \operatorname{Res}_{s=\rho_{H_{r}}} \beta(s) .
$$

Now an examination of the results in [1k, I1, I2] shows that the function $\beta(s)$ has the form

$$
\zeta_{*}\left(s-\left(\rho_{H_{r}}-1\right)\right) \quad \text { or } \quad \zeta_{*}\left(2 s-\left(2 \rho_{H_{r}}-1\right)\right),
$$

where $*=E$ or $F$. On the other hand, $\operatorname{Res}_{s=1} L\left(s, M_{r}\right) / L\left(s, H_{r}\right)$ is equal to $\operatorname{Res}_{s=1} \zeta_{*}(s)$. Hence we see that

$$
\operatorname{Res}_{s=1}\left(\frac{L\left(s, M_{r}\right)}{L\left(s, H_{r}\right)}\right) / \operatorname{Res}_{s=\rho_{H_{r}}} \beta(s)=1 \quad \text { or } \quad 2
$$

in the two respective cases, so that

$$
\alpha=\operatorname{Res}_{s=\rho_{H_{r}}} c_{r}(s) \quad \text { or } 2 \cdot \operatorname{Res}_{s=\rho_{H_{r}}} c_{r}(s) .
$$

Indeed, a careful examination shows that the second case happens precisely when

$$
H_{0}=\left\{\begin{array}{l}
\mathrm{O}_{1} \text { or trivial, when } \epsilon_{0}=1 ; \\
\text { trivial, when } \epsilon_{0}=0
\end{array}\right.
$$

One can then recast the expressions for $\alpha$ into a uniform formula:

$$
\alpha=\frac{\tau\left(H_{r}\right)}{\tau\left(H_{0}\right)} \cdot \# \pi_{0}\left(H_{r} / H_{0}\right) \cdot \operatorname{Res}_{s=\rho_{H_{r}}} c_{r}(s) .
$$

By part (i) of the lemma, we then deduce that

$$
\alpha=\frac{\tau\left(H_{r}\right)}{\tau\left(H_{0}\right)} \cdot \kappa_{r}
$$

as desired.

3.4. Measure constants. Motivated by Lemma 3.4(ii), for any maximal parabolic subgroup $P\left(X_{r-r^{\prime}}\right)$ of $H_{r}$, with Levi subgroup $\operatorname{GL}\left(X_{r-r^{\prime}}\right) \times H_{r^{\prime}}$, we define a constant $\kappa_{r, r^{\prime}}$ by the requirement that

$$
\frac{1}{\tau\left(H_{r}\right)} \cdot d h=\kappa_{r, r^{\prime}} \cdot \frac{1}{\tau\left(H_{r^{\prime}}\right)} \cdot d m \cdot d n \cdot d k
$$

where $d m$ and $d n$ are the Tamagawa measures of $M\left(X_{r-r^{\prime}}\right)$ and $N\left(X_{r-r^{\prime}}\right)$, respectively. In particular, $\kappa_{r}=\kappa_{r, 0}$. 
3.5. Regularized theta integral. The regularized theta integral is defined to be the function

$$
B^{n, r}(s, \phi)(g)=\frac{1}{\tau\left(H_{r}\right) \cdot \kappa_{r} \cdot P_{n, r}(s)} \cdot \int_{\left[H_{r}\right]} \theta_{n, r}\left(\omega_{n, r}(z) \phi\right)(g, h) E_{H_{r}}(s, h) d h .
$$

When $r=0$ and $m_{0}>0$, we set $B^{n, 0}(s, \phi)(g)=I_{n, 0}(\phi)(g)$ by convention.

The integral converges absolutely at all points $s$ where $E_{H_{r}}(s, h)$ is holomorphic, and defines a meromorphic function of $s$ (for fixed $\phi$ ). We note that the functional equation (3.3) implies

$$
B^{n, r}(s, \phi)=c_{r}(s) \cdot B^{n, r}(-s, \phi) .
$$

We can now explain why the extra condition $r \leq n$ as in (3.2) is necessary. Indeed, if this condition is not satisfied, one cannot hope to regularize the theta integral in the same way as above. Otherwise, one may integrate the regularized theta kernel against the Eisenstein series associated to the family of principal series representations induced from the minimal parabolic subgroup of $H_{r}$; this gives a meromorphic function in $r$ complex variables whose iterated residue at a specific point is the regularized theta lift of the constant function of $H_{r}$. If this meromorphic function is not identically zero, then a Zariski open set of these minimal principal series representations will have a nonzero theta lift to $G_{n}$. For this to happen, it is necessary that $n \geq r$.

3.6. First and Second term range. The analytic behavior of $B^{n, r}(s, \phi)$ at the point of interest $s=\rho_{H_{r}}$ is described as follows:

Lemma 3.8. (i) If $m \leq d(n)$, then $B^{n, r}(s, \phi)$ has a pole of order at most 1 at $s=\rho_{H_{r}}$.

(ii) If $d(n)<m \leq 2 \cdot d(n)$ (under the conditions in (3.1)), then $P_{n, r}(s)$ has a simple zero at $s=\rho_{H_{r}}$ and $B^{n, r}(s, \phi)$ has a pole of order at most 2 at $s=\rho_{H_{r}}$.

Proof. See [KR5, Lemma 5.5.6], [12, Lemma 2.2] and [T1, §2].

Because the analytic behavior of $B^{n, r}(s, \phi)$ at $s=\rho_{H_{r}}$ differs for different ranges of the pair $(n, m)$, we introduce the following terminology outside the Weil's convergent range:

$$
\begin{gathered}
m<d(n): \text { the first term range; } \\
m=d(n): \text { the boundary case; } \\
d(n)<m \leq 2 \cdot d(n): \text { the second term range. }
\end{gathered}
$$

Observe that if $V_{r}$ and $V_{r^{\prime}}$ are complementary spaces, then $\left(W_{n}, V_{r}\right)$ is in the first term range if and only if $\left(W_{n}, V_{r^{\prime}}\right)$ is in the second term range. Moreover, in the boundary case, we have $V_{r}=V_{r^{\prime}}$. Note also that when $m>2 \cdot d(n)$, one is automatically in the Weil's convergent range.

3.7. Laurent expansion. In the first term range and the boundary case, we may thus consider the Laurent expansion at $s=\rho_{H_{r}}$ :

$$
B^{n, r}(s, \phi)=\frac{B_{-1}^{n, r}(\phi)}{s-\rho_{H_{r}}}+B_{0}^{n, r}(\phi)+\cdots
$$

whereas in the second term range, we have

$$
B^{n, r}(s, \phi)=\frac{B_{-2}^{n, r}(\phi)}{\left(s-\rho_{H_{r}}\right)^{2}}+\frac{B_{-1}^{n, r}(\phi)}{s-\rho_{H_{r}}}+B_{0}^{n, r}(\phi)+\cdots .
$$

The functions $B_{d}^{n, r}(\phi)$ are automorphic forms on $G_{n}$ and the linear map $\omega_{n, r} \rightarrow \mathcal{A}\left(G_{n}\right)$ given by $\phi \mapsto$ $B_{d}^{n, r}(\phi)$ is $G_{n}(\mathbb{A})$-equivariant. Moreover, if $B_{d}^{n, r}$ is the leading term in the Laurent expansion, it is $H_{r}(\mathbb{A})$-invariant. 
The purpose of the regularized Siegel-Weil formula is to give an alternative construction of the automorphic forms $B_{-1}^{n, r}(\phi)$ and $B_{-2}^{n, r}(\phi)$.

3.8. Non-Siegel Eisenstein Series. We now recall how the regularized theta integral $B^{n, r}(s, \phi)$ can be expressed as a non-Siegel Eisenstein series on $G_{n}$. Namely, one can massage the definition of $B^{n, r}(s, \phi)$ by unfolding the auxiliary Eisenstein series $E_{H_{r}}(s,-)$. As shown in [KR5, §5.5, p.48-50] and using Lemma 3.4(ii), one obtains:

\section{Proposition 3.9.}

$$
B^{n, r}(s, \phi)=E^{n, r}\left(s, f^{n, r}\left(s, \pi_{K_{H_{r}}}(\phi)\right)\right) .
$$

The following explains the notation in the proposition:

- $E^{n, r}(s,-)$ refers to the Eisenstein series associated to the family of induced representations

$$
I_{r}^{n}(s, \chi)=\operatorname{Ind}_{Q\left(Y_{r}\right)(\mathbb{A})}^{G_{n}(\mathbb{A})}(\chi \circ \operatorname{det})\left|\operatorname{det}_{Y_{r}}\right|^{s} \otimes \Theta_{n-r, 0}\left(V_{0}\right)
$$

where we recall that the Levi factor of $Q\left(Y_{r}\right)$ is $L\left(Y_{r}\right) \cong \mathrm{GL}\left(Y_{r}\right) \times G_{n-r}$ and $\Theta_{n-r, 0}\left(V_{0}\right)$ is as defined in 3.1. This is what we call the non-Siegel Eisenstein series (though if $r=n$, it is of course a Siegel-Eisenstein series).

- $\pi_{K_{H_{r}}}$ is the projection operator onto the $K_{H_{r}}$-fixed subspace, defined by

$$
\pi_{K_{H_{r}}}(\phi)=\int_{K_{H_{r}}} \omega_{n, r}(k)(\phi) d k .
$$

- For $\phi \in \mathcal{S}\left(Y_{n}^{*} \otimes V_{r}\right)(\mathbb{A})$,

$$
f^{n, r}(s, \phi) \in I_{r}^{n}(s, \chi)
$$

is a meromorphic section defined for $\operatorname{Re}(s) \gg 0$ by

$$
\begin{aligned}
f^{n, r}(s, \phi)(g) & =\int_{\mathrm{GL}\left(X_{r}\right)(\mathbb{A})} I_{n-r, 0}\left(\omega_{n, r}(g, a) \mathcal{F}_{n, r}(\phi)\left(\beta_{0}\right)(0,-)\right) \cdot\left|\operatorname{det}_{X_{r}}(a)\right|^{s-\rho_{H}} d a \\
& =\int_{\mathrm{GL}\left(X_{r}\right)(\mathbb{A})} I_{n-r, 0}\left(\omega_{n, r}(g) \mathcal{F}_{n, r}(\phi)\left(\beta_{0} \circ a\right)(0,-)\right) \cdot\left|\operatorname{det}_{X_{r}}(a)\right|^{s+n-\rho_{H}} d a .
\end{aligned}
$$

Here we note that $\mathcal{F}_{n, r}(\phi)$ is a Schwartz function on $X_{r}^{*} \otimes W_{n}=\operatorname{Hom}\left(X_{r}, W_{n}\right)$ taking values in

$$
\mathcal{S}\left(Y_{n}^{*} \otimes V_{0}\right)(\mathbb{A})=\mathcal{S}\left(Y_{r}^{*} \otimes V_{0}\right)(\mathbb{A}) \otimes \mathcal{S}\left(Y_{n-r}^{\prime *} \otimes V_{0}\right)(\mathbb{A}),
$$

and

is defined by

$$
\beta_{0} \in \operatorname{Hom}\left(X_{r}, W_{n}\right)
$$

so that

$$
\beta_{0}\left(x_{i}\right)=y_{i} \quad \text { for } i=1, \ldots, r,
$$

$$
\mathcal{F}_{n, r}(\phi)\left(\beta_{0} \circ a\right)(0,-) \in \mathcal{S}\left(Y_{n-r}^{\prime *} \otimes V_{0}\right)(\mathbb{A}) .
$$

The integral defining $f^{n, r}(s, \phi)$ converges when

$$
\operatorname{Re}(s)>\frac{m}{2}-\frac{2 n-r+\epsilon_{0}}{2}
$$

and extends to a meromorphic section of $I_{r}^{n}(s, \chi)$ (since it is basically a Tate-Godement-Jacquet zeta integral, as we explain in the next section). When $r=0$ and $m_{0}>0$, we set $f^{n, 0}(s, \phi)(g)=I_{n, 0}(\phi)(g)$ by convention. 
Thus we have expressed $B^{n, r}(s, \phi)$ as an Eisenstein series on $G_{n}$ associated to a meromorphic (nonstandard) section of a family of non-Siegel principal series representations. However, our ultimate goal is to relate the first two Laurent coefficients of $B^{n, r}(s, \phi)$ to the Laurent coefficients of a Siegel Eisenstein series.

\section{The section $f^{n, r}(s, \phi)$.}

In this section, we shall establish some important properties of the section $f^{n, r}(s,-)$ defined in 3.8 , which will play a crucial role in the proof of the second term identity.

4.1. Tate-Godement-Jacquet Zeta Integrals. We recall briefly the global theory of the Tate-GodementJacquet zeta integral developed in GJ]. If $\phi \in \mathcal{S}\left(M_{r \times r}\right)\left(\mathbb{A}_{E}\right)$, then the Tate-Godement-Jacquet zeta integral (associated to the trivial representation of $\mathrm{GL}_{r}\left(\mathbb{A}_{E}\right)$ ) is:

$$
Z_{r}(s, \phi):=\int_{\mathrm{GL}_{r}\left(\mathbb{A}_{E}\right)} \phi(A) \cdot|\operatorname{det}(A)|^{s} d A .
$$

It converges for $\operatorname{Re}(s)>r$ and has meromorphic continuation to $\mathbb{C}$. Moreover, it satisfies a functional equation

$$
Z_{r}(s, \phi)=Z_{r}(r-s, \hat{\phi})
$$

where $\hat{\phi}$ is the Fourier transform of $\phi$ relative to the Haar measure on $M_{r \times r}\left(\mathbb{A}_{E}\right)$ determined by $\psi$ and the trace form on $M_{r \times r}\left(\mathbb{A}_{E}\right)$. And $Z_{r}(s, \phi)$ has simple poles at $s=0$ and $r$, with $\operatorname{Res}_{s=0} Z_{r}(s, \phi)=-\phi(0)$ and $\operatorname{Res}_{s=r} Z_{r}(s, \phi)=\hat{\phi}(0)$.

4.2. The section $\mathfrak{f}^{n, r}(s, \phi)$. It will be convenient to express elements of $Y_{n}^{*} \otimes V_{r}$ as $3 \times 2$ matrices corresponding to the decompositions

$$
Y_{n}^{*}=Y_{r}^{*} \oplus Y_{n-r}^{\prime *} \quad \text { and } \quad V_{r}=X_{r} \oplus V_{0} \oplus X_{r}^{*},
$$

so the first column of the matrix has entries from $Y_{r}^{*} \otimes X_{r}, Y_{r}^{*} \otimes V_{0}$ and $Y_{r}^{*} \otimes X_{r}^{*}$ in this order, and the second column has entries from $Y_{n-r}^{\prime *} \otimes X_{r}, Y_{n-r}^{\prime *} \otimes V_{0}$ and $Y_{n-r}^{\prime *} \otimes X_{r}^{*}$.

Then by definition

$$
\begin{aligned}
& f^{n, r}(s, \phi)(g)= I_{n-r, 0}\left(\int_{\mathrm{GL}\left(X_{r}\right)(\mathbb{A})} \int_{\left(Y^{\prime *}{ }_{n-r} \otimes X_{r}\right)(\mathbb{A})} \int_{\left(Y_{r}^{*} \otimes X_{r}\right)(\mathbb{A})}\right. \\
&\left.\omega_{n, r}(g) \phi\left(\begin{array}{cc}
X_{1} & X_{2} \\
0 & - \\
0 & 0
\end{array}\right) \cdot \psi\left(\operatorname{Tr}\left(X_{1} A\right)\right) \cdot|\operatorname{det}(A)|^{s+n-\rho_{H_{r}}} d X_{1} d X_{2} d A\right) .
\end{aligned}
$$

Now we set:

$$
\begin{aligned}
\mathfrak{f}^{n, r}(s, \phi)(g)(-):= & \int_{\mathrm{GL}\left(X_{r}\right)(\mathbb{A})} \int_{\left(Y^{\prime *}{ }_{n-r} \otimes X_{r}\right)(\mathbb{A})} \int_{\left(Y_{r}^{*} \otimes X_{r}\right)(\mathbb{A})} \\
& \omega_{n, r}(g) \phi\left(\begin{array}{cc}
X_{1} & X_{2} \\
0 & - \\
0 & 0
\end{array}\right) \cdot \psi\left(\operatorname{Tr}\left(X_{1} A\right)\right) \cdot|\operatorname{det}(A)|^{s+n-\rho_{H_{r}}} d X_{1} d X_{2} d A .
\end{aligned}
$$

In other words, $\mathfrak{f}^{n, r}(s, \phi)$ is defined as $f^{n, r}(s, \phi)$ but with the anisotropic theta integral $I_{n-r, 0}$ suppressed. Then we note that

$$
\mathfrak{f}^{n, r}(s, \phi) \in \operatorname{Ind}_{Q\left(Y_{r}\right)(\mathbb{A})}^{G_{n}(\mathbb{A})}(\chi \circ \operatorname{det}) \cdot|\operatorname{det}|^{s} \otimes \omega_{n-r, 0},
$$

with $\omega_{n-r, 0}$ realized on $\mathcal{S}\left(Y_{n-r}^{\prime *} \otimes V_{0}\right)(\mathbb{A})$, and

$$
f^{n, r}(s, \phi)(g)=I_{n-r, 0}\left(\mathfrak{f}^{n, r}(s, \phi)(g)\right) .
$$


Observe that the formation of $\mathfrak{f}^{n, r}(s,-)$ is basically the Tate-Godemont-Jacquet zeta integral of a partial Fourier transform of $\phi$ (in the coordinate $X_{1}$ ). Since the anisotropic theta integral presents no convergence issues, we see that the meromorphic continuation of $f^{n, r}(s, \phi)$ follows immediately from the analytic theory of the Tate-Godement-Jacquet zeta integral. Moreover, applying the functional equation (4.1) for the Tate-Godement-Jacquet zeta integral, we deduce:

Lemma 4.3. One has:

$$
\mathfrak{f}^{n, r}(s, \phi)(g)(-)=\int_{\mathrm{GL}\left(X_{r}\right)(\mathbb{A})} \int_{\left(Y_{n-r}^{\prime *} \otimes X_{r}\right)(\mathbb{A})} \omega_{n, r}(g) \phi\left(\begin{array}{cc}
A & X_{2} \\
0 & - \\
0 & 0
\end{array}\right) \cdot|\operatorname{det}(A)|^{-s+r-n+\rho_{H_{r}}} d X_{2} d A .
$$

This is a somewhat simple expression to work with, as we shall see in a moment.

4.3. Restriction and Ikeda map. Next we consider the restriction of the section $f^{n+1, r}(s, \phi)$ from $G_{n+1}$ to $G_{n}$; this restriction turns out to be related to the Ikeda map $\mathrm{Ik}^{n, r, r-1}$. More precisely, fix $\phi_{1} \in \mathcal{S}\left(Y_{1}^{*} \otimes V_{r}\right)(\mathbb{A})$ satisfying:

- $\phi_{1}(0)=1$;

- $\phi_{1}$ is $K_{H_{r}}$-invariant, so that $\pi_{K_{H_{r}}} \phi_{1}=\phi_{1}$.

For any $\phi \in \mathcal{S}\left(Y_{n}^{\prime *} \otimes V_{r}\right)(\mathbb{A})$, we set

$$
\tilde{\phi}:=\phi_{1} \otimes \phi \in \mathcal{S}\left(Y_{n+1}^{*} \otimes V_{r}\right)(\mathbb{A}) .
$$

Then

$$
\pi_{K_{H_{r}}}(\tilde{\phi})=\phi_{1} \otimes \pi_{K_{H_{r}}} \phi
$$

We consider the restriction of $f^{n+1, r}(s, \tilde{\phi})$ to the subgroup $G_{n} \subset G_{n+1}$, where $G_{n}$ is the isometry group of the nondegenerate subspace $W_{n}=\left\langle y_{2}, \ldots, y_{n+1}, y_{n+1}^{*}, \ldots, y_{2}^{*}\right\rangle \subset W_{n+1}$. With these conventions, the result is:

Proposition 4.4. Suppose $r \geq 1$ and further assume $m_{0}>0$ when $r=1$. We have:

$$
\left.f^{n+1, r}\left(s, \pi_{K_{H_{r}}} \tilde{\phi}\right)\right|_{G_{n}}=\alpha_{r} \cdot Z_{1}\left(-s-(n+1-r)+\rho_{H_{r}}, \phi_{1}\right) \cdot f^{n, r-1}\left(s+\frac{1}{2}, \mathrm{Ik}^{n, r, r-1}\left(\pi_{K_{H_{r}}} \phi\right)\right) .
$$

Here $Z_{1}\left(s, \phi_{1}\right)$ is the Tate zeta integral

$$
Z_{1}\left(s, \phi_{1}\right)=\int_{\mathbb{A}_{E}^{\times}} \phi_{1}\left(t y_{1}^{*} \otimes x_{1}\right) \cdot|t|^{s} d t
$$

and $\alpha_{r}$ is a scalar defined by 4.5) below.

Proof. By (4.2), it suffices to show the identity with $\mathfrak{f}^{n+1, r}(s, \phi)$ in place of $f^{n+1, r}(s, \phi)$ (since $I_{(n+1)-r, 0}=$ $\left.I_{n-(r-1), 0}\right)$. Now for $g \in G_{n}(\mathbb{A})$, Lemma 4.3 gives

$$
\begin{aligned}
& f^{n+1, r}\left(s, \pi_{K_{H_{r}}} \tilde{\phi}\right)(g)(-) \\
= & \int_{\mathrm{GL}\left(X_{r}\right)(\mathbb{A})} \int_{\left(Y^{\prime *}{ }_{n+1-r} \otimes X_{r}\right)(\mathbb{A})}\left(\omega_{n+1, r}(g) \pi_{K_{H_{r}}} \tilde{\phi}\right)\left(\begin{array}{cc}
A & Y \\
0 & - \\
0 & 0
\end{array}\right) \cdot|\operatorname{det}(A)|^{-s-(n+1-r)+\rho_{H_{r}}} d Y d A \\
= & \int_{\mathrm{GL}\left(X_{r}\right)(\mathbb{A})} \int_{\left(Y^{\prime *}{ }_{n+1-r} \otimes X_{r}\right)(\mathbb{A})} \phi_{1} \otimes \omega_{n, r}(g) \pi_{K_{H_{r}}} \phi\left(\begin{array}{cc}
A & Y \\
0 & - \\
0 & 0
\end{array}\right) \cdot|\operatorname{det}(A)|^{-s-(n+1-r)+\rho_{H_{r}}} d Y d A,
\end{aligned}
$$

where, for the second equality, we have used the fact that

$$
\omega_{n+1, r}(g) \pi_{K_{H_{r}}} \tilde{\phi}=\phi_{1} \otimes \omega_{n, r}(g) \pi_{K_{H_{r}}} \phi
$$


since $G_{n}(\mathbb{A})$ acts trivially on $\phi_{1}$.

Assume first that $r=1$ and $m_{0}>0$. Since $\phi_{1}$ is a function on the first column of the matrix in the integrand, we can just decompose the integral as

$$
\begin{aligned}
& \mathfrak{f}^{n+1, r}\left(s, \pi_{K_{H_{r}}} \tilde{\phi}\right)(g)(-) \\
= & \left(\int_{\mathrm{GL}\left(X_{r}\right)(\mathbb{A})} \phi_{1}\left(\begin{array}{c}
A \\
0 \\
0
\end{array}\right) \cdot|\operatorname{det}(A)|^{-s-(n+1-r)+\rho_{H_{r}}} d A\right) \cdot\left(\int_{\left(Y_{n}^{\prime *} \otimes X_{r}\right)(\mathbb{A})}\left(\omega_{n, r}(g) \pi_{K_{H_{r}}} \tilde{\phi}\right)\left(\begin{array}{c}
Y \\
- \\
0
\end{array}\right) d Y\right) .
\end{aligned}
$$

Noting that the first factor is the Tate integral $Z_{1}\left(-s-(n+1-r)+\rho_{H_{r}}, \phi_{1}\right)$ and the second one is $\mathfrak{f}^{n, r-1}\left(s+\frac{1}{2}, \mathrm{Ik}^{n, r, r-1}\left(\pi_{K_{H_{r}}} \phi\right)\right)(g)(-)$, we see that the proposition holds with $\alpha_{1}=1$.

Assume now that $r>1$. To extract the first column of $A$, we use the Iwasawa decomposition on $\mathrm{GL}\left(X_{r}\right)(\mathbb{A}) \cong \mathrm{GL}_{r}\left(\mathbb{A}_{E}\right)$. Namely, we have

$$
A=k \cdot\left(\begin{array}{ll}
1 & u \\
0 & 1
\end{array}\right) \cdot\left(\begin{array}{cc}
t & 0 \\
0 & B
\end{array}\right)=k \cdot\left(\begin{array}{cc}
t & u B \\
0 & B
\end{array}\right)
$$

with

- $t \in \mathbb{A}_{E}^{\times}$

- $u \in\left(x_{1} \otimes X^{\prime *-1}\right)(\mathbb{A}) \cong \mathbb{A}_{E}^{r-1}$

- $B \in \mathrm{GL}\left(X_{r-1}^{\prime}\right)(\mathbb{A}) \cong \mathrm{GL}_{r-1}\left(\mathbb{A}_{E}\right)$

- $k$ is an element in a maximal compact subgroup $K=K_{H_{r}} \cap \mathrm{GL}\left(X_{r}\right)(\mathbb{A})$ of $\mathrm{GL}\left(X_{r}\right)(\mathbb{A})$.

Accordingly, we have a constant $\alpha_{r}$ such that

$$
\int_{\mathrm{GL}_{r}\left(\mathbb{A}_{E}\right)} \varphi(A) d A=\alpha_{r} \cdot \int_{\mathbb{A}_{E}^{\times}} \int_{\mathrm{GL}_{r-1}\left(\mathbb{A}_{E}\right)} \int_{\mathbb{A}_{E}^{r-1}} \int_{K} \varphi\left(k \cdot\left(\begin{array}{cc}
t & u B \\
0 & B
\end{array}\right)\right) d t d B d u d k
$$

for any $\varphi \in C_{c}^{\infty}\left(\mathrm{GL}_{r}\left(\mathbb{A}_{E}\right)\right)$. Indeed, by [12, Lemma 9.1], one has:

$$
\alpha_{r}=\frac{\operatorname{Res}_{s=1} \zeta_{E}(s)}{\zeta_{E}(r)}
$$

Since the function $\pi_{K_{H_{r}}} \tilde{\phi}$ is $K_{H_{r}}$-invariant, the integral over $d k$ simply gives the value 1 and thus disappears. Hence, after using the Iwasawa decomposition, we obtain:

$$
\begin{aligned}
& \mathfrak{f}^{n+1, r}\left(s, \pi_{K_{H_{r}}} \tilde{\phi}\right)(g)(-)=\alpha_{r} \cdot \int_{t} \int_{B} \int_{u} \int_{Y} \phi_{1} \otimes \omega_{n, r}(g) \pi_{K_{H_{r}}} \phi\left(\begin{array}{ccc}
t & u B & Y \\
0 & B & Y \\
0 & - \\
0 & 0
\end{array}\right) \\
&|t|_{E}^{-s-(n+1-r)+\rho_{H_{r}}} \cdot|\operatorname{det}(B)|^{-s-(n+1-r)+\rho_{H_{r}}} d t d B d u d Y,
\end{aligned}
$$

where $t \in \mathbb{A}_{E}^{\times}, B \in \mathrm{GL}_{r-1}\left(\mathbb{A}_{E}\right), u \in \mathbb{A}_{E}^{r-1}$ and $Y \in\left(Y_{n-r}^{\prime *} \otimes X_{r}\right)(\mathbb{A})$.

Noting that $\phi_{1}$ is a function of the first column of the $m \times(n+1)$ matrix in the integrand, we see that the above integral is equal to the product of

$$
\alpha_{r} \cdot \int_{t \in \mathbb{A}_{E}^{\times}} \phi_{1}\left(\begin{array}{l}
t \\
0 \\
0 \\
0
\end{array}\right) \cdot|t|_{E}^{-s-(n+1-r)+\rho_{H_{r}}} d t
$$


and

$$
\int_{u} \int_{B} \int_{Y}\left(\omega_{n, r}(g) \pi_{K_{H}} \phi\right)\left(\begin{array}{cc}
u & Y \\
0 & - \\
0 & 0
\end{array}\right) \cdot|\operatorname{det}(B)|^{-s-1-(n+1-r)+\rho_{H_{r}}} d Y d B d u,
$$

where we have made the substitution $u \mapsto u B^{-1}$. Now observe that (4.6) is equal to the Tate zeta integral

$$
\alpha_{r} \cdot Z_{1}\left(-s-(n+1-r)+\rho_{H_{r}}, \phi_{1}\right)
$$

where $\phi_{1}$ is actually the function defined by $t \mapsto \phi_{1}\left(t y_{1}^{*} \otimes x_{1}\right)$.

On the other hand, in (4.7), we may write the $m \times n$ matrix as:

$$
\left(\begin{array}{cc}
u & \mathrm{Y} \\
B & - \\
0 & - \\
0 & 0
\end{array}\right)=\left(\begin{array}{cc}
B^{z} & Y^{\dagger} \\
0 & - \\
0 & 0
\end{array}\right)
$$

with $z \in \mathbb{A}_{E}^{n}$ occupying the first row of the matrix and $Y^{\dagger}$ a $r \times(n-r)$ matrix. Then, on replacing the integrals over $u$ and $Y$ by integrals over $z$ and $Y^{\dagger}$, and noting that

$$
\rho_{H_{r}}=\frac{m-r-\epsilon_{0}}{2}=\frac{m_{0}+r-\epsilon_{0}}{2}=\rho_{H_{r-1}}+\frac{1}{2},
$$

we see that (4.7) is equal to

$$
\int_{B} \int_{z} \int_{Y^{\dagger}}\left(\omega_{n, r}(g) \pi_{K_{H}} \phi\right)\left(\begin{array}{cc}
z & Y^{\dagger} \\
0 & - \\
0 & 0
\end{array}\right) \cdot|\operatorname{det}(B)|^{-\left(s+\frac{1}{2}\right)+r-1-n+\rho_{H_{r-1}}} d z d Y^{\dagger} d B
$$

which is nothing but

$$
\mathfrak{f}^{n, r-1}\left(s+\frac{1}{2}, \operatorname{Ik}^{n, r, r-1}\left(\pi_{K_{H_{r}}} \phi\right)\right)(g)(-) .
$$

This completes the proof of the proposition.

Remark 4.8. It is natural to extend the definition of $f^{n, r}(s, \phi)$ and $f^{n, r}(s, \phi)$ by regarding them as functions on $G_{n} \times H_{r}$, rather than simply functions on $G_{n}$. More precisely, we set

$$
\mathfrak{F}^{n, r}(s, \phi)(g, h)(-)=\int_{\mathrm{GL}\left(X_{r}\right)(\mathbb{A})} \omega_{n, r}(g, h)\left(\mathcal{F}_{n, r}(\phi)\left(\beta_{0} \circ a\right)(0,-)\right) \cdot\left|\operatorname{det}_{X_{r}}(a)\right|^{s+n-\rho_{H}} d a,
$$

and

$$
F^{n, r}(s, \phi)(g, h):=I_{n-r, 0}\left(\mathfrak{F}^{n, r}(s, \phi)(g, h)\right)
$$

Then

Observe that

$$
\left.\mathfrak{F}^{n, r}(s, \phi)\right|_{G_{n}}=\mathfrak{f}^{n, r}(s, \phi) \quad \text { and }\left.\quad F^{n, r}(s, \phi)\right|_{G_{n}}=f^{n, r}(s, \phi)
$$

$$
\left.\mathfrak{F}^{n, r}(s, \phi) \in \operatorname{Ind}_{Q\left(Y_{r}\right)(\mathbb{A}) \times P\left(X_{r}\right)(\mathbb{A})}^{G_{n}(\mathbb{A}) \times H_{r}(\mathbb{A})}\left(\left(\chi \circ \operatorname{det}_{Y_{r}}\right) \cdot\left|\operatorname{det}_{Y_{r}}\right|^{s} \otimes\left|\operatorname{det}_{X_{r}}\right|^{-s}\right) \otimes \omega_{n-r, 0}\right),
$$

and

$$
F^{n, r}(s, \phi) \in I_{r}^{n}(s, \chi) \otimes I_{H_{r}}(-s) .
$$

Thus,

$$
F^{n, r}(s,-): \omega_{n, r} \longrightarrow I_{r}^{n}(s, \chi) \otimes I_{H_{r}}(-s)
$$

is a meromorphic family of $G_{n}(\mathbb{A}) \times H_{r}(\mathbb{A})$-equivariant maps which explicitly realizes the Howe duality correspondence. 


\section{Siegel Principal Series}

In this section, we review the theory of the Siegel principal series representation and its relation to the Weil representation over a local field. Hence throughout this section, we let $k$ be a (possibly archimedean) local field and let $L$ be $k$ if $\epsilon_{0} \neq 0$ and a quadratic étale algebra over $k$ if $\epsilon_{0}=0$, so $L$ is either a quadratic extension of $k$ or $k \times k$. We assume the spaces $W_{n}$ and $V_{r}$ are defined over $L$. Thus, unlike the global case, we include in our discussion the split quadratic algebra $L=k \times k$, in which case $G_{n}(k) \cong \mathrm{GL}_{2 n}(k)$ and $H_{r}(k) \cong \mathrm{GL}_{m}(k)$. In addition, in this section, $\chi$ is any unitary character on $L^{\times}$.

We shall consider the degenerate principal series representation

$$
I_{n}^{n}(s, \chi):=\operatorname{Ind}_{Q\left(Y_{n}\right)(k)}^{G_{n}(k)}(\chi \circ \operatorname{det}) \cdot|\operatorname{det}|^{s} .
$$

Here we recall that when $G_{n}(k)=\operatorname{Mp}_{2 n}(k)$, then

$$
I_{n}^{n}(s, \chi)=\operatorname{Ind}_{Q\left(Y_{n}\right)(k)}^{G_{n}(k)}\left(\chi_{\psi} \circ \operatorname{det}\right) \cdot(\chi \circ \operatorname{det}) \cdot|\operatorname{det}|^{s} .
$$

5.1. Degenerate principal series: Non-archimedean case. We first assume that $k$ is non-archimedean. The following proposition summarizes the reducibility points of $I_{n}^{n}(s, \chi)$.

Proposition 5.1. Let $k$ be non-archimedean.

(i) The representation $I_{n}^{n}(s, \chi)$ is multiplicity-free.

(ii) Assume that $s \in \mathbb{R}$ and $\chi$ is unitary. Then $I_{n}^{n}(s, \chi)$ is reducible precisely in the situations given in the following table.

\begin{tabular}{|c|c|}
\hline$\epsilon_{0}=0, L \neq k \times k$ & $\left.\chi\right|_{k^{\times}}=\chi_{L}^{m}$ and $s=\frac{m-d(n)}{2}$ with $0 \leq m \leq 2 d(n)$ \\
\hline$\epsilon_{0}=0, L=k \times k$ & $\left.\chi\right|_{k^{\times}}=1$ and $s=\frac{m-d(n)}{2}$ with $0 \leq m \leq 2 d(n)$ and $m \neq n$ \\
\hline$\epsilon_{0}=-1$ & $\chi^{2}=1$ and $s=\frac{m-d(n)}{2}$ with $m$ even and $0 \leq m \leq 2 d(n)$ \\
\hline$\epsilon_{0}=1, G_{n}=\mathrm{Mp}_{2 n}$ & $\chi^{2}=1$ and $s=\frac{m-d(n)}{2}$ with $m$ odd and $1 \leq m \leq 2 n+1$ \\
\hline$\epsilon_{0}=1, G_{n}=\mathrm{Sp}_{2 n}$ & (a) $\chi^{2}=1$ but $\chi \neq 1$ and $s=\frac{m-d(n)}{2}$, with $m$ even $2 \leq m \leq 2 n$ \\
& or \\
& (b) $\chi=1$ and $s=\frac{m-d(n)}{2}$ with $m$ even and $0 \leq m \leq 2 n+2$ \\
\hline
\end{tabular}

Proof. See KR4, KS, S2, Y1.

Observe that the points of reducibility are essentially the points

$$
s_{m, n}=\frac{m-d(n)}{2}, \quad 0 \leq m \leq 2 d(n)
$$

with some extra conditions in the various cases:

- when $\epsilon_{0}=1, m$ is even when $G_{n}$ is symplectic, and $m$ is odd when $G_{n}$ is metaplectic; moreover, there is a quadratic space $V$ over $k$ of dimension $m$ and discriminant corresponding to the quadratic character $\chi$;

- when $\epsilon_{0}=-1$, there is a symplectic space $V$ of dimension $m$ (so that $m$ is even);

- when $\epsilon_{0}=0$ and $L=k \times k, m \neq n$. 
5.2. Set $X_{n}$. For both archimedean and non-archimedean $k$, let us define

$$
X_{n}:=\{(s, \chi):(s, \chi) \text { appears in the table of Proposition [5.1 }\}
$$

If $k$ is non-archimedean, this set is precisely the set of all $(s, \chi)$ with $s \in \mathbb{R}$ and $\chi$ unitary such that $I_{n}^{n}(s, \chi)$ is reducible. If $k$ is archimedean, as we will see, $I_{n}^{n}(s, \chi)$ is also reducible for $(s, \chi) \in X_{n}$, though there are more points of reducibility which will play no role in this paper.

5.3. Siegel-Weil sections. Assume $k$ is archimedean or non-archimedean. The structure of $I_{n}^{n}(s, \chi)$ at the points of reducibility is intimately connected with the theory of local theta correspondence. To describe this, we recall the following construction. For the local Weil representation $\omega_{n, r}$ realized on $\mathcal{S}\left(Y_{n}^{*} \otimes V_{r}\right)(k)$, we set

$$
\Phi^{n, r}(\phi)(g)=\left(\omega_{n, r}(g) \phi\right)(0), \quad \text { for } \phi \in \mathcal{S}\left(Y_{n}^{*} \otimes V_{r}\right)(k)
$$

This defines an $H_{r}(k)$-invariant and $G_{n}(k)$-equivariant map

$$
\Phi^{n, r}: \mathcal{S}\left(Y_{n}^{*} \otimes V_{r}\right)(k) \longrightarrow I_{n}^{n}\left(\frac{m-d(n)}{2}, \chi\right),
$$

with $\chi=\chi_{V_{0}}$. We call the space of standard sections associated to the image of $\Phi^{n, r}$ the space of Siegel-Weil sections associated to $V_{r}$.

5.4. The representation $R_{n}\left(V_{r}\right)$. Assume again that $k$ is archimedean or non-archimedean. Since $\Phi^{n, r}$ is $H_{r}(k)$-invariant, it factors through the maximal $H_{r}(k)$-invariant quotient $R_{n}\left(V_{r}\right)$ of $\omega_{n, r}$. It was shown by Rallis that one has

$$
\Phi^{n, r}: \mathcal{S}\left(Y_{n}^{*} \otimes V_{r}\right)(k) \rightarrow R_{n}\left(V_{r}\right) \hookrightarrow I_{n}^{n}\left(\frac{m-d(n)}{2}, \chi\right),
$$

so that the image of $\Phi^{n, r}$ is isomorphic to $R_{n}\left(V_{r}\right)$. Thus $R_{n}\left(V_{r}\right)$ is a submodule of $I_{n}^{n}\left(\frac{m-d(n)}{2}, \chi\right)$.

5.5. Structure of $I_{n}^{n}(s, \chi)$. Assume $k$ is non-archimedean. We can now describe the module structure of $I_{n}^{n}(s, \chi)$ at a point of reducibility. For a point of reducibility $s_{m, n}=\frac{m-d(n)}{2}$ as given in Proposition 5.1, we let $V$ and $V^{\prime}$ be given as follows:

- when $\epsilon_{0}=0$ and $L \neq k \times k, V$ and $V^{\prime}$ are the two $\epsilon$-Hermitian spaces of dimension $m$ with $\operatorname{disc}(V) \in N_{L / k} L^{\times}$and $\operatorname{disc}\left(V^{\prime}\right) \notin N_{L / k} L^{\times}$

- when $\epsilon_{0}=0$ and $L=k \times k, V$ is the unique $\epsilon$-Hermitian space of dimension $m$ and we set $V^{\prime}=0$;

- when $\epsilon_{0}=-1, V$ is the unique symplectic space of dimension $m$;

- when $\epsilon_{0}=1, V$ and $V^{\prime}$ are the two quadratic spaces of dimension $m$ with $V$ having Hasse-Witt invariant 1 and $V^{\prime}$ having Hasse-Witt invariant -1 (here, a split quadratic space has Hasse-Witt invariant 1).

Note that $V^{\prime}$ is not defined when $\epsilon_{0}=-1$ and may not exist in the other cases when $m$ is too small. We have seen that $R_{n}(V)$ and $R_{n}\left(V^{\prime}\right)$ are submodules of $I_{n}^{n}\left(s_{m, n}, \chi\right)$. Here, when $\epsilon_{0}=-1$, so that $G_{n} \cong \mathrm{O}_{n, n}$, we interpret

$$
R_{n}\left(V^{\prime}\right)=R_{n}(V) \otimes \operatorname{det}_{G_{n}},
$$

and when $V^{\prime}$ does not exist in the other cases, we interpret $R_{n}\left(V^{\prime}\right)=0$. With these notations, we have:

Proposition 5.2. Assume that $k$ is non-archimedean.

(i) If $s_{m, n}=\frac{m-d(n)}{2}<0$, then $R_{n}(V)$ and $R_{n}\left(V^{\prime}\right)$ are irreducible unitary and $R_{n}(V) \oplus R_{n}\left(V^{\prime}\right)$ is the maximal semisimple submodule of $I_{n}^{n}\left(s_{m, n}, \chi\right)$. The quotient

$$
I_{n}^{n}\left(s_{m, n}, \chi\right) /\left(R_{n}(V) \oplus R_{n}\left(V^{\prime}\right)\right)
$$

is irreducible. 
(ii) If $s_{m, n}=0$, then

$$
I_{n}^{n}(0, \chi)=R_{n}(V) \oplus R_{n}\left(V^{\prime}\right) .
$$

(iii) If $s_{m, n}>0$, then

$$
I_{n}^{n}\left(s_{m, n}, \chi\right)=R_{n}(V)+R_{n}\left(V^{\prime}\right)
$$

and the intersection

$$
R_{n}(V) \cap R_{n}\left(V^{\prime}\right)
$$

is the unique irreducible submodule of $I_{n}^{n}\left(s_{m, n}, \chi\right)$. If $V_{c}$ and $V_{c}^{\prime}$ are the complementary spaces of $V$ and $V^{\prime}$ (relative to $W_{n}$ ), so that $\operatorname{dim} V+\operatorname{dim} V_{c}=2 \cdot d(n)$ with $\operatorname{dim} V_{c}<\operatorname{dim} V$, then one has

$$
R_{n}(V) / R_{n}(V) \cap R_{n}\left(V^{\prime}\right) \cong R_{n}\left(V_{c}\right) \quad \text { and } \quad R_{n}\left(V^{\prime}\right) / R_{n}(V) \cap R_{n}\left(V^{\prime}\right) \cong R_{n}\left(V_{c}^{\prime}\right) .
$$

Thus there is a short exact sequence

$$
0 \longrightarrow R_{n}(V) \cap R_{n}\left(V^{\prime}\right) \longrightarrow I_{n}^{n}\left(s_{m, n}, \chi\right) \longrightarrow R_{n}\left(V_{c}\right) \oplus R_{n}\left(V_{c}^{\prime}\right) \longrightarrow 0 .
$$

Proof. See [KR4, KS, S2, Y1].

5.6. Archimedean case. When $F=\mathbb{R}$ or $\mathbb{C}$, the structure of the degenerate principal series $I_{n}^{n}(s, \chi)$ has also been completely determined in [L1, L2, [HL, [Lo, Appendix A], and [LZ3, LZ4. Moreover, the relation of the submodules to the theory of local theta correspondence was determined in [LZ1, LZ2, LZ3, [ZZ4. The results are too intricate to state here, but we note the following, which is sufficient for our purposes:

Proposition 5.3. Assume that $k=\mathbb{R}$ or $\mathbb{C}$.

(i) As a representation of the maximal compact subgroup $K$ of $G_{n}(k)$, the representation $I_{n}^{n}(s, \chi)$ is independent of $s$ and is multiplicity-free.

(ii) For $\left(s_{m, n}, \chi\right) \in X_{n}$ with $s_{m, n} \geq 0$,

$$
I_{n}^{n}\left(s_{m, n}, \chi\right)=\sum_{V} R_{n}(V)
$$

where the sum runs over all $\epsilon$-Hermitian spaces $V$ of dimension $m$ with the following additional condition and convention:

- if $\epsilon_{0}=1, \chi_{V}=\chi$;

- if $\epsilon_{0}=-1$, so that there is a unique symplectic space $V$ of dimension $m$, the $R H S$ is $R_{n}(V)+$ $R_{n}(V) \otimes \operatorname{det}$.

So, for example, when $k=\mathbb{C}$ and $\left(s_{m, n}, \chi\right) \in X_{n}$, one has:

$$
I_{n}^{n}\left(s_{m, n}, \chi\right)=\left\{\begin{array}{l}
R_{n}(V) \text { if } \epsilon_{0} \neq-1 \\
R_{n}(V)+R_{n}(V) \otimes \text { det, if } \epsilon_{0}=-1 .
\end{array}\right.
$$

5.7. Local intertwining operator. We now let $k$ be an arbitrary local field. Define

$$
c= \begin{cases}\text { the identity, } & \text { if } L=k ; \\ \text { the non-trivial element in } \operatorname{Aut}(L / k), & \text { if } L \text { is an étale quadratic } k \text {-algebra. }\end{cases}
$$

For each character $\chi$ on $L^{\times}$, we define $\chi^{c}$ to be the character on $L^{\times}$such that $\chi^{c}(x)=\chi(c(x))$.

There is a standard intertwining operator

$$
M_{n}(s, \chi): I_{n}^{n}(s, \chi) \longrightarrow I_{n}^{n}\left(-s,\left(\chi^{c}\right)^{-1}\right) .
$$


One may normalize this intertwining operator following [LR] (see also [GI2, §8]). We will not recall the precise definition of this normalization here, but simply note that the normalized operator $M^{*}(s, \chi)$ satisfies the functional equation

$$
M_{n}^{*}\left(-s,\left(\chi^{c}\right)^{-1}\right) \circ M_{n}^{*}(s, \chi)=1 .
$$

We note that for $(s, \chi) \in X_{n},\left(\chi^{c}\right)^{-1}=\chi$.

We are particularly interested in the behavior of $M_{n}^{*}(s, \chi)$ at $s=0$.

Lemma 5.4. (i) The normalized intertwining operator $M_{n}^{*}(s, \chi)$ is holomorphic at $s=0$, and satisfies

$$
M_{n}^{*}(0, \chi)^{2}=1 .
$$

(ii) If $k$ is non-archimedean, $M_{n}^{*}(0, \chi)$ acts as +1 on $R_{n}(V)$ and -1 on $R_{n}\left(V^{\prime}\right)$.

(iii) The derivative $M_{n}^{* \prime}(0, \chi)$ commutes with $M_{n}^{*}(0, \chi)$ and preserves each irreducible summand of $I_{n}^{n}(0, \chi)$.

Proof. (i) The holomorphy of $M_{n}^{*}(s, \chi)$ at $s=0$ follows from the fact that $M_{n}^{*}(-s, \chi) \circ M_{n}^{*}(s, \chi)=1$ and $I_{n}^{n}(0, \chi)$ is semisimple. The holomorphy implies the remaining part of (i).

(ii) That it indeed acts as in the lemma was checked in [GT] when $\epsilon_{0}=-1$, GS, Lemma 7.2] when $\epsilon_{0}=1$ and [HKS, [KS] when $\epsilon_{0}=0$; it is responsible for the phenomenon known as the local theta dichotomy, which was shown in [HKS] and [GS].

(iii) Differentiating the identity $M_{n}^{*}(-s, \chi) \circ M_{n}^{*}(s, \chi)=1$ and evaluating at $s=0$, we obtain

$$
M_{n}^{* \prime}(0, \chi) \circ M_{n}^{*}(0, \chi)=M_{n}^{*}(0, \chi) \circ M_{n}^{* \prime}(0, \chi),
$$

as desired. If $k$ is non-archimedean, the two irreducible summands of $I_{n}^{n}(0, \chi)$ are different eigenspaces for the action of $M_{n}^{*}(0, \chi)$ and thus each summand is preserved by the derivative $M_{n}^{* \prime}(0, \chi)$. In the archimedean case, we observe that $M_{n}^{* \prime}(0, \chi)$ is $K$-equivariant. Indeed, one has

$$
M_{n}^{*}(s, \chi) \circ I_{n}^{n}(s, \chi)(g)=I_{n}^{n}(-s, \chi)(g) \circ M_{n}^{*}(s, \chi)
$$

for all $g \in G_{n}$, and for $k \in K$, the operator $I_{n}^{n}(s, \chi)(k)$ is independent of $s$. Thus differentiating the above equation and evaluating at $s=0$, we obtain

$$
M_{n}^{* \prime}(0, \chi) \circ I_{n}^{n}(0, \chi)(k)=I_{n}^{n}(0, \chi)(k) \circ M_{n}^{* \prime}(0, \chi)
$$

for all $k \in K$. Now the desired result follows from the fact that $I_{n}^{n}(0, \chi)$ is $K$-multiplicity-free, as we noted in Prop. 5.3(i).

\section{Siegel Eisenstein Series}

In this section, let us return to the setting of the number field $F$, and consider the global analog of the previous section. In particular, we consider the global Siegel principal series representation $I_{n}^{n}(s, \chi)$ of $G_{n}(\mathbb{A})$. Here, we take $\chi=\chi_{V_{r}}=\chi_{V_{0}}$ to be a Hecke character fixed as in (2.8), depending on the $\epsilon$-Hermitian space $V_{r}$. In particular, $\chi^{c}=\chi^{-1}$. Also we let the set

$$
X_{n}(\chi):=\{s \in \mathbb{C}: s \text { appears in the table of Proposition } 5.1 \text { for the fixed } \chi \cdot\}
$$


6.1. Siegel Eisenstein series. For a standard section $\Phi_{s} \in I_{n}^{n}(s, \chi)$, we consider the Siegel Eisenstein series defined for $\operatorname{Re}(s) \gg 0$ by

$$
E(s, \Phi)(g):=\sum_{\gamma \in Q\left(Y_{n}\right)(F) \backslash G_{n}(F)} \Phi_{s}(\gamma g)
$$

for $g \in G_{n}(\mathbb{A})$. Sometimes we write

$$
E(s, \Phi)=E^{n, n}(s, \Phi)
$$

when we want to emphasize the rank of the group. It admits a meromorphic continuation to $\mathbb{C}$.

The following proposition, due to Kudla-Rallis [KR3] and V. Tan [T2, summarizes the analytic properties of the Siegel Eisenstein series.

Proposition 6.1. Exclude the case when $G_{n}=\mathrm{O}_{1,1}$. For $\operatorname{Re}(s) \geq 0, E(s, \Phi)$ is holomorphic except at $s=s_{m, n} \in X_{n}(\chi)$ with $s_{m, n}>0$. At these points, $E(s, \Phi)$ has a pole of order at most 1 .

In view of the above proposition, we may consider the Laurent expansion of $E(s, \Phi)$ at $s=s_{m, n}>0$ :

$$
E\left(s, \Phi_{s}\right)=\frac{E_{-1, s_{m, n}}(\Phi)}{s-s_{m, n}}+E_{0, s_{m, n}}(\Phi)+\cdots
$$

whereas at $s=0$, one has

$$
E\left(s, \Phi_{s}\right)=E_{0, s_{m, n}}(\Phi)+E_{1, s_{m, n}}(\Phi) \cdot s+\cdots .
$$

Here we many view each $E_{d, s_{m, n}}$ as a linear map $I_{n}^{n}(s, \chi) \rightarrow \mathcal{A}\left(G_{n}\right)$. Viewing the Laurent coefficients in this way, we note that when $s_{m, n}>0, E_{-1, s_{m, n}}$ is $G_{n}(\mathbb{A})$-equivariant, where $E_{0, s_{m, n}}$ is $G_{n}(\mathbb{A})$-equivariant modulo the image of $E_{-1, s_{m, n}}$. When $s_{m, n}=0$, the analogous statement holds for the first and second Laurent coefficients $E_{0, s_{m, n}}$ and $E_{1, s_{m, n}}$.

6.2. Global Siegel-Weil sections. Analogously to the local case, we may introduce the notion of a Siegel-Weil section. For an $\epsilon$-Hermitian space $V_{r}$ of dimension $m=m_{0}+2 r$ over $E$, we have

$$
\Phi^{n, r}: \mathcal{S}\left(Y_{n}^{*} \otimes V_{r}\right)(\mathbb{A}) \longrightarrow I_{n}^{n}\left(\frac{m-d(n)}{2}, \chi\right)
$$

defined by

$$
\Phi^{n, r}(\phi)(g)=\omega_{n, r}(g) \phi(0) .
$$

Its image is isomorphic to the maximal $H\left(V_{r}\right)(\mathbb{A})$-invariant quotient of $\omega_{n, r}$ :

$$
R_{n}\left(V_{r}\right)=\otimes_{v} R_{n}\left(V_{r, v}\right) .
$$

6.3. Coherent vs. Incoherent. Now suppose that $s_{m, n} \in X_{n}(\chi)$ and $s_{m, n} \geq 0$. To describe the structure of $I_{n}^{n}\left(s_{m, n}, \chi\right)$, let us make the following definition following Kudla-Rallis [KR5].

(1) Assume $\epsilon_{0} \neq-1$. Let $V_{r}^{c}$ be the space complementary to $V_{r}$ with respect to $W_{n}$ (so $\operatorname{dim} V_{r}^{c}=m^{\prime} \leq m$ is such that $\left.m+m^{\prime}=2 d(n)\right)$. Let $\mathcal{C}=\left\{U_{v}\right\}$ be a collection of $\epsilon$-Hermitian spaces $U_{v}$ over $F_{v}$ such that

- $\operatorname{dim} U_{v}=m^{\prime}$ for all $v$;

- $U_{v}=V_{r, v}^{c}$ for almost all $v$;

- if $\epsilon_{0}=1$, then $\chi_{U_{v}}=\chi_{v}$ for all $v$.

Then we call the collection $\mathcal{C}$ coherent if there is a global $\epsilon$-Hermitian space $V$ over $F$ such that $V \otimes F_{v}=U_{v}$. Otherwise we call the collection $\mathcal{C}$ incoherent. 
(2) Assume $\epsilon_{0}=-1$, so $G_{n}=\mathrm{O}_{n, n}$. Let $\mathcal{C}=\left\{\eta_{v}\right\}$ be a collection of characters of $G_{n}$ with $\eta_{v}=\mathbf{1}$ or det and such that $\eta_{v}=\mathbf{1}$ for almost all $v$. Then we call the collection $\mathcal{C}$ coherent if $\eta_{v}=\operatorname{det}$ for an even number of $v$, or equivalently $\otimes_{v} \eta_{v}$ is an automorphic determinant character. Otherwise we call $\mathcal{C}$ incoherent.

For each $\mathcal{C}=\left\{U_{v}\right\}$ or $\left\{\eta_{v}\right\}$, we define

$$
R_{n}(\mathcal{C})= \begin{cases}\otimes_{v}^{\prime} R_{n}\left(U_{v}\right), & \text { if } \epsilon_{0} \neq-1 \\ \otimes_{v}^{\prime} R_{n}\left(V_{r, v}^{c}\right) \otimes \eta_{v}, & \text { if } \epsilon_{0}=-1\end{cases}
$$

By Proposition 5.2 and 5.3 , we see that the maximal semisimple quotient of $I_{n}^{n}\left(s_{m, n}, \chi\right)$ is given by

$$
\bigoplus_{\mathcal{C}} R_{n}(\mathcal{C})
$$

where the sum runs over all the collections $\mathcal{C}$ (coherent or not) as defined above.

As a special case, when $s_{m, n}=0$, so that $m=m^{\prime}=d(n)$, the degenerate principal series $I(0, \chi)$ is semisimple and one has:

$$
I_{n}^{n}(0, \chi)=\left(\bigoplus_{\text {coherent } \mathcal{C}} R_{n}(\mathcal{C})\right) \oplus\left(\bigoplus_{\text {incoherent } \mathcal{C}} R_{n}(\mathcal{C})\right) .
$$

We call the first summand the coherent submodule and the second the incoherent submodule.

6.4. The leading term of $E\left(s, \Phi_{s}\right)$. The following proposition describes the image of the leading term $E_{-1, s_{m, n}}$ or $E_{0, s_{m, n}}$ (see KR3, KR5, GT, T2, Y4).

Proposition 6.2. (i) When $s_{m, n}>0$,

$$
\operatorname{Im} E_{-1, s_{m, n}} \cong \bigoplus_{\mathcal{C}} R_{n}(\mathcal{C}),
$$

where $\mathcal{C}$ runs over coherent collections.

(ii) When $s_{m, n}=0$,

$$
\operatorname{Im} E_{0, s_{m, n}} \cong \bigoplus_{\mathcal{C}} R_{n}(\mathcal{C})
$$

where $\mathcal{C}$ is as in (i).

6.5. Intertwining operator. One has the standard global intertwining operator

$$
M_{n}(s, \chi): I_{n}^{n}(s, \chi) \longrightarrow I_{n}^{n}(-s, \chi),
$$

which satisfies

$$
M_{n}(-s, \chi) \circ M_{n}(s, \chi)=1 .
$$

The normalization for the local intertwining operators is such that one has:

$$
M_{n}(s, \chi)=\otimes_{v} M_{n, v}^{*}\left(s, \chi_{v}\right) .
$$

Moreover, one has the functional equation

$$
E(s, \Phi)=E\left(-s, M_{n}(s, \chi) \Phi\right) .
$$

We collect some of the important properties of the intertwining operator $M_{n}(s, \chi)$ that we need.

Lemma 6.3. (i) $M_{n}(s, \chi)$ is holomorphic at $s=0$ and $M_{n}(0, \chi)^{2}=1$.

(ii) $M_{n}(0, \chi)$ acts as +1 on the coherent submodule of $I_{n}^{n}(0, \chi)$ and -1 on the incoherent submodule. 
(iii) The derivative $M_{n}^{\prime}(0, \chi)$ commutes with $M_{n}(0, \chi)$ and preserves each irreducible submodule $R_{n}(\mathcal{C}) \subset$ $I_{n}^{n}(0, \chi)$.

Proof. (i) This is shown in the same way as Lemma 5.4(i).

(ii) The case for $G_{n}=\mathrm{O}_{n, n}$ is [GT, Lemma 7.4 (i)]; so we assume $\epsilon_{0} \neq-1$ here. First note that $I_{n}^{n}(0, \chi)$ is multiplicity free and semisimple, and hence part (i) of the lemma implies that $M_{n}(0, \chi)$ acts on each irreducible summand as $\eta= \pm 1$. Suppose $\Phi \in I_{n}^{s}(0, \chi)$ is in an irreducible subspace of the coherent submodule. By the functional equation of the Eisenstein series, we have $E(0, \Phi)=E(0, \eta \Phi)$. But Proposition 6.2 (ii) implies that $E(0, \Phi) \neq 0$, which implies $\eta=1$. Next suppose $\Phi$ is in an irreducible subspace of the incoherent submodule. We may assume that $\Phi$ is factorizable as $\Phi=\otimes_{v} \Phi_{v}$, with $\Phi_{v} \in R_{n}\left(U_{v}\right)$ and

$$
M_{n, v}^{*}\left(0, \chi_{v}\right) \Phi_{v}=\eta_{v} \Phi_{v}
$$

for some signs $\eta_{v}$. Now pick a finite place $v_{0}$ inert in $E$, and define

$$
\mathcal{C}=\left\{U_{v_{0}}^{\prime}\right\} \cup\left\{U_{v}: v \neq v_{0}\right\}
$$

where $U_{v_{0}}^{\prime}$ is as defined as in the beginning of 95.5 so that $U_{v_{0}}^{\prime} \neq U_{v_{0}}$. Then $\mathcal{C}$ is a coherent collection. If we pick a non-zero $\Phi_{v_{0}}^{\prime} \in R_{n}\left(U_{v_{0}}^{\prime}\right)$, then Lemma 5.4 implies that

$$
M_{n, v_{0}}^{*}\left(0, \chi_{v_{0}}\right) \Phi_{v_{0}}=-\eta_{v_{0}} \Phi_{v_{0}} .
$$

Now let

$$
\Phi^{\prime}=\Phi_{v_{0}}^{\prime} \otimes\left(\otimes_{v \neq v_{0}} \Phi_{v}\right) .
$$

This is in the coherent submodule, and hence

$$
M_{n}(0, \chi) \Phi^{\prime}=\Phi^{\prime}
$$

But we also have

$$
M_{n}(0, \chi) \Phi^{\prime}=\left(-\eta_{v_{0}} \prod_{v \neq v_{0}} \eta_{v}\right) \cdot \Phi^{\prime}=-\eta \cdot \Phi^{\prime} .
$$

Hence $\eta=-1$ as desired. It is worth noting that the proof of (ii) is somewhat indirect as its local counterpart Lemma 5.4(ii) has only been shown in the non-archimedean case.

(iii) The first statement is proved in the same way as the analogous statement in Lemma 5.4(iii). The second part also follows from the local analogues (Lemma 5.4(iii)) by arguing semi-locally. Namely, we may assume that each section $\Phi \in R_{n}(\mathcal{C})$ can be factorized as $\Phi=\otimes_{v \in S} \Phi_{v} \otimes \Phi_{S}$, where $\Phi_{S}=\otimes_{v \notin S} \Phi_{v}$ and $S$ is a finite set of places such that $\Phi_{v}$ is spherical for $v \notin S$. Accordingly, we may decompose the intertwining operator as $M_{n}(0, \chi)=\otimes_{v \in S} M_{n, v}^{*}\left(0, \chi_{v}\right) \otimes M_{n, S}^{*}\left(0, \chi_{S}\right)$, where $M_{n, S}^{*}\left(0, \chi_{S}\right)$ is defined analogously to $\Phi_{S}$. By Leibnitz rule, the derivative $M_{n}^{\prime}(0, \chi)$ is computed as

$$
\begin{aligned}
M_{n}^{\prime}(0, \chi) & =\left(\sum_{v_{0} \in S} M_{n, v_{0}}^{* \prime}\left(0, \chi_{v_{0}}\right) \otimes\left(\underset{\substack{\otimes \\
v \neq 0_{0}, v \in S}}{\otimes} M_{n, v}^{*}\left(0, \chi_{v}\right)\right)\right) \otimes M_{n, S}^{*}\left(0, \chi_{S}\right) \\
& +\underset{v \in S}{\otimes} M_{n, v}^{*}\left(0, \chi_{v}\right) \otimes M_{n, S}^{* \prime}\left(0, \chi_{S}\right) .
\end{aligned}
$$

For the unramified part, one sees that $M_{n, S}^{*}\left(0, \chi_{S}\right) \Phi_{S}=c_{S} \Phi_{S}$ and $M_{n, S}^{* \prime}\left(0, \chi_{S}\right) \Phi_{S}=d_{S} \Phi_{S}$ for some scalars $c_{S}$ and $d_{S}$. For each $v \in S, M_{n, v}^{*}\left(0, \chi_{v}\right)$ acts as \pm 1 on the local component of $R_{n}(\mathcal{C})$, and the derivative $M_{n, v}^{* \prime}\left(0, \chi_{v}\right)$ preserves the component by Lemma 5.4 (iii). Hence if one applies $\Phi$ to the above expression of the derivative $M_{n}^{\prime}(0, \chi)$, one sees that $M_{n}^{\prime}(0, \chi) \Phi \in R_{n}(\mathcal{C})$. 
6.6. The Laurent coefficients $A_{d}^{n, r}$. Now we consider the Siegel-Weil sections arising from $V_{r}$ (with $\left.\operatorname{dim}_{E} V_{r}=m\right)$. At the point $s_{m, n} \in X_{n}(\chi)$ with $s_{m, n} \geq 0$, we have the Laurent expansion

$$
E\left(s, \Phi^{n, r}(\phi)\right)=\frac{A_{-1}^{n, r}(\phi)}{s-s_{m, n}}+A_{0}^{n, r}(\phi)+\cdots \quad \text { if } s_{m, n}>0
$$

or

$$
E\left(s, \Phi^{n, r}(\phi)\right)=A_{0}^{n, r}(\phi)+A_{1}^{n, r}(\phi) \cdot s+\cdots \quad \text { if } s_{m, n}=0 .
$$

Thus $A_{d}^{n, r}$ is viewed as a linear map:

$$
A_{d}^{n, r}=E_{d, s_{m, n}} \circ \Phi^{n, r}: \mathcal{S}\left(Y_{n}^{*} \otimes V_{r}\right)(\mathbb{A}) \longrightarrow \mathcal{A}\left(G_{n}\right) .
$$

Let us close this section with the following proposition, which we do not need for this paper, but is interesting to take note of nevertheless.

Proposition 6.4. If $A_{d}^{n, r}$ denotes the leading Laurent coefficient above (so $d=0$ or -1 ), then $A_{d}^{n, r}$ is $G_{n}(\mathbb{A})$-equivariant. If $A_{d+1}^{n, r}$ is the second Laurent coefficient, then

$$
A_{d+1}^{n, r}: \mathcal{S}\left(Y_{n}^{*} \otimes V_{r}\right)(\mathbb{A}) \longrightarrow \mathcal{A}\left(G_{n}\right) / \operatorname{Im} A_{d}^{n, r}
$$

is $G_{n}(\mathbb{A})$-intertwining.

Proof. To ease the notation we denote $I_{n}^{n}(s, \chi)$ simply by $I(s, \chi)$. Since

$$
A_{j}^{n, r}=E_{j, s_{m, n}} \circ \Phi^{n, r}
$$

we see that $A_{d}^{n, r}$ is $G_{n}(\mathbb{A})$-equivariant but $A_{d+1}^{n, r}$ is $G(\mathbb{A})$-equivariant modulo the image of $E_{d, s_{m, n}}$. Indeed, for any $g \in G(\mathbb{A})$, one has

$$
A_{d+1}^{n, r}\left(\omega_{n, r}(g) \phi\right)=g \cdot A_{d+1}^{n, r}(\phi)+E_{d, s_{m, n}}\left(I^{\prime}\left(s_{m, n}, \chi\right)(g) \Phi^{n, r}(\phi)\right)
$$

where

$$
I^{\prime}\left(s_{m, n}, \chi\right)(g)=\left.\frac{d}{d s} I(s, \chi)(g)\right|_{s=s_{m, n}} \in \operatorname{End}_{K}\left(I\left(s_{m, n}, \chi\right)\right) .
$$

Here let us note that End $_{K}$ indicates the space of $K$-equivariant operators. Thus we would like to show that

$$
E_{d, s_{m, n}}\left(I^{\prime}\left(s_{m, n}, \chi\right)(g) \Phi^{n, r}(\phi)\right) \in \operatorname{Im} A_{d}^{n, r} .
$$

Now we need to argue semi-locally as we did in the proof of Lemma 6.3 (iii). Namely fix $\Phi^{n, r}(\phi)$ so that it is factored as

$$
\Phi^{n, r}(\phi)=\otimes_{v \in S} \Phi_{v}^{n, r}\left(\phi_{v}\right) \otimes \Phi_{S}^{n, r}\left(\phi_{S}\right)
$$

for a finite set $S$ of places such that for each $v \notin S, \Phi_{v}^{n, r}\left(\phi_{v}\right)$ is spherical. Also since $g \in G_{n}(\mathbb{A})$ is fixed, we assume $S$ is large enough so that $g_{v}$ belongs to a hyperspecial maximal compact subgroup for $v \notin S$. By Leibnitz rule, one has

$$
\begin{aligned}
& I^{\prime}\left(s_{m, n}, \chi\right)(g)\left(\Phi^{n, r}(\phi)\right) \\
= & \left(\sum_{v_{0} \in S} I_{v_{0}}^{\prime}\left(s_{m, n}, \chi_{v_{0}}\right)\left(g_{v_{0}}\right) \Phi_{v_{0}}^{n, r}\left(\phi_{v_{0}}\right) \otimes\left(\prod_{\substack{v \neq v_{0} \\
v \in S}} I_{v}\left(s_{m, n}, \chi_{v}\right)\left(g_{v}\right) \Phi_{v}^{n, r}\left(\phi_{v}\right)\right)\right) \otimes I_{S}\left(s_{m, n}, \chi_{S}\right)\left(g_{S}\right) \Phi_{S}^{n, r}\left(\phi_{S}\right) \\
& +\left(\prod_{v \in S} I_{v}\left(s_{m, n}, \chi_{v}\right)\left(g_{v}\right) \Phi_{v}^{n, r}\left(\phi_{v}\right)\right) \otimes I_{S}^{\prime}\left(s_{m, n}, \chi_{S}\right)\left(g_{S}\right) \Phi_{S}^{n, r}\left(\phi_{S}\right) .
\end{aligned}
$$


Note that for any $v \notin S, I_{v}^{\prime}\left(s_{m, n}, \chi_{v}\right)\left(g_{v}\right) \Phi_{v}^{n, r}\left(\phi_{v}\right)=0$ because $I_{v}\left(s, \chi_{v}\right)\left(g_{v}\right)$ is independent of $s$ (since $g_{v}$ lies in the hyperspecial maximal compact subgroup). Hence in the above expression of the derivative, the second term is simply zero. Thus the derivative is written as

$$
I^{\prime}\left(s_{m, n}, \chi\right)(g)\left(\Phi^{n, r}(\phi)\right)=\sum_{v_{0} \in S} I_{v_{0}}^{\prime}\left(s_{m, n}, \chi_{v_{0}}\right)\left(g_{v_{0}}\right) \Phi_{v_{0}}^{n, r}\left(\phi_{v_{0}}\right) \otimes\left(\prod_{v \neq v_{0}} I_{v}\left(s_{m, n}, \chi_{v}\right)\left(g_{v}\right) \Phi_{v}^{n, r}\left(\phi_{v}\right)\right) .
$$

Now we claim that for any $v_{0}$,

$$
I_{v_{0}}^{\prime}\left(s_{m, n}, \chi_{v_{0}}\right)\left(g_{v_{0}}\right) \Phi_{v_{0}}^{n, r}\left(\phi_{v_{0}}\right) \otimes\left(\prod_{v \neq v_{0}} I_{v}\left(s_{m, n}, \chi_{v}\right)\left(g_{v}\right) \Phi_{v}^{n, r}\left(\phi_{v}\right)\right)
$$

is either incoherent or lies in $\operatorname{Im} \Phi^{n, r}$, so that

$$
E_{d, s_{m, n}}\left(I_{v_{0}}^{\prime}\left(s_{m, n}, \chi_{v_{0}}\right)\left(g_{v_{0}}\right) \Phi_{v_{0}}^{n, r}\left(\phi_{v_{0}}\right) \otimes\left(\prod_{v \neq v_{0}} I_{v}\left(s_{m, n}, \chi_{v}\right)\left(g_{v}\right) \Phi_{v}^{n, r}\left(\phi_{v}\right)\right)\right) \in \operatorname{Im} A_{d}^{n, r} .
$$

This implies the proposition (on recalling Proposition 6.2).

For each $v, I_{v}\left(s_{m, n}, \chi_{v}\right)\left(g_{v}\right) \Phi_{v}^{n, r}\left(\phi_{v}\right) \in \operatorname{Im} \Phi_{v}^{n, r}$ because $\Phi_{v}^{n, r}$ is $G_{v}\left(F_{v}\right)$-equivariant. Hence it remains to examine the derivative term at $v_{0}$. In what follows, the subscript $v_{0}$ is omitted because everything is over $F_{v_{0}}$.

(a) When $v_{0}$ is non-archimedean, the claim is obvious, since $I^{\prime}\left(s_{m, n}, \chi\right)(g) \Phi^{n, r}(\phi)$ either lies in $\operatorname{Im} \Phi^{n, r}$ or else projects to $R_{n}(U)$ where $U$ is of complementary dimension to $V_{r}$ but belongs to a different Witt tower.

(b) When $v_{0}$ is complex, there is nothing to show since $I\left(s_{m, n}, \chi\right)=R_{n}(V)$ by [LZ3].

(c) When $v_{0}$ is real and $\epsilon_{0}=-1$, the same argument as in the non-archimedean case works, since the structure of $I\left(s_{m, n}, \chi\right)$ here is similar to that in the non-archimedean case. However, when $\epsilon_{0} \neq-1$, the claim is not so obvious, since the module structure of $I_{n}^{n}\left(s_{m, n}, \chi\right)$ is more complicated. Since we do not need this proposition in the rest of the paper, we shall only give a sketch of the argument in these cases.

Recall first that $I_{n}^{n}\left(s_{m, n}, \chi\right)$ is $K$-multiplicity-free (Prop. $\left.5.3(\mathrm{i})\right)$ and the operator $I\left(s_{m, n}, \chi\right)(k)$ is independent of $s$, so that $I^{\prime}\left(s_{m, n}, \chi\right)(k) \Phi^{n, r}(\phi) \in \operatorname{Im} \Phi^{n, r}$. Thus the main point is to verify the claim for elements $X \in \mathfrak{p}$, where $\mathfrak{g}=\mathfrak{k} \oplus \mathfrak{p}$ is the Cartan decomposition of the Lie algebra $\mathfrak{g}$ of $G_{n}$.

In [L1, L2], the module structure of $I(s, \chi)$ is completely determined by studying how the K-types in $I(s, \chi)$ are moved around by the operators $I(s, \chi)(X)$ with $X \in \mathfrak{p}$. In particular, the transition coefficients from one $K$-type to another are precisely determined and turn out to be linear functions of $s$. Reducibility occurs at those $s$ when some of the transition coefficients vanish. Since the derivatives of these transition coefficients are nonzero constants, we deduce from these results in [L1, L2 that the derivative $I^{\prime}\left(s_{m, n}, \chi\right)(X)$ moves the $K$-types in $I\left(s_{m, n}, \chi\right)$ in the same way as $I(s, \chi)(X)$ does for generic $s$.

By examining the different cases, one arrives at the following simple conclusion. If $\Phi$ belongs to a particular $K$-type $\tau$ of $I\left(s_{m, n}, \chi\right)$, then $I^{\prime}\left(s_{m, n}, \chi\right)(X)(\Phi)$ can only have components belonging to $K$ types $\tau^{\prime}$ which are "adjacent" to $\tau$. Here, $\tau^{\prime}$ is adjacent to $\tau$ if its highest weight differs from $\tau$ in only one coordinate and in that coordinate, the difference is the minimum allowed in the given case.

Now consider the natural projection map

$$
\pi: I\left(s_{m, n}, \chi\right) \longrightarrow \oplus_{U} R_{n}(U)
$$


SIEGEL-WEIL AND RALLIS

where $\operatorname{dim} U$ is complementary to $\operatorname{dim} V_{r}$. The above discussion implies that if $\pi(\Phi) \in R_{n}\left(U_{0}\right)$, then $\pi\left(I^{\prime}\left(s_{m, n}, \chi\right)(X)(\Phi)\right)$ can only have nonzero components in $R_{n}(U)$ for those $U$ whose signature is adjacent to that of $U_{0}$ in the obvious sense. Thus, for example, in the case $\epsilon_{0}=0$, if the signature of $U_{0}$ is $(p, q)$, then the adjacent signatures are $(p, q),(p+1, q-1)$ and $(p-1, q+1)$. This proves the desired claim and hence the proposition.

\section{The Siegel-Weil formula}

In this section, we recall the known cases of the Siegel-Weil formula.

7.1. Anisotropic case. We first consider the case $r=0$, i.e. the pair $\left(W_{n}, V_{0}\right)$, so $H_{0}=H\left(V_{0}\right)$ is anisotropic. In this case, the Siegel-Weil formula is due to Weil We1], Kudla-Rallis [KR1, Ichino [13] and Yamana $\mathrm{Y} 2$.

Theorem 7.1. For $\phi \in \mathcal{S}\left(Y_{n}^{*} \otimes V_{0}\right)(\mathbb{A})$, the Eisenstein series $E\left(s, \Phi^{n, 0}(\phi)\right)$ is holomorphic at $s=s_{m, n}=$ $(m-d(n)) / 2$ and

with

$$
E\left(s_{m, n}, \Phi^{n, 0}(\phi)\right)=c_{m, n} \cdot \frac{\tau\left(H_{0}\right)}{[E: F]} \cdot I_{n, 0}(\phi)
$$

$$
c_{m, n}= \begin{cases}1, & \text { if } s_{m, n}>0, \\ 2, & \text { if } s_{m, n} \leq 0 .\end{cases}
$$

Moreover, the term $\tau\left(H_{0}\right) /[E: F]=1$, except when $H_{0}=\mathrm{O}_{1}$ in which case it is equal to $1 / 2$.

More precisely, Weil We1 established the case when $m>2 \cdot d(n)$, Kudla-Rallis KR1 established the case when $\epsilon_{0}=1$, Ichino [I3] showed the case when $\epsilon_{0}=0$ and $d(n)<m \leq 2 \cdot d(n)$, and Yamana [Y2] completed the case $\epsilon_{0}=0$ and $m \leq d(n)$. There is nothing to check when $\epsilon_{0}=-1$.

For later purposes, it is necessary for us to compute the constant term of $I_{n, 0}(\phi)$ along the maximal parabolic subgroup $Q_{1}=Q\left(Y_{1}\right)$. We have:

Proposition 7.2. For

$$
\phi \in \mathcal{S}\left(Y_{n}^{*} \otimes V_{0}\right)(\mathbb{A})=\mathcal{S}\left(y_{1}^{*} \otimes V_{0}\right)(\mathbb{A}) \otimes \mathcal{S}\left(Y_{n-1}^{\prime *} \otimes V_{0}\right)(\mathbb{A})
$$

we have

$$
\left.I_{n, 0}(\phi)_{U_{1}}\right|_{\mathrm{GL}\left(Y_{1}\right)(\mathbb{A}) \times G_{n-1}(\mathbb{A})}=\chi \cdot|-|^{\frac{1}{2} m_{0}} \otimes I_{n-1,0}(\phi(0,-)) .
$$

Proof. If $V_{0}=0$, there is nothing to prove. Thus we assume that $V_{0} \neq 0$; in particular, $V_{0}$ is not symplectic and so $G_{n}$ is not an orthogonal group. This implies that the unipotent radical $U_{1}$ of $Q_{1}$ sits in a short exact sequence

$$
1 \longrightarrow Z_{1} \longrightarrow U_{1} \longrightarrow U_{1} / Z_{1} \longrightarrow 1
$$

with

$$
Z_{1} \cong\left\{\epsilon \text {-Hermitian forms on } Y_{1}^{*}\right\} \cong \mathbb{G}_{a} .
$$

Now we compute the constant term. For $g=\left(t, g_{0}\right) \in \mathrm{GL}\left(Y_{1}\right)(\mathbb{A}) \times G_{n-1}(\mathbb{A})$, we have:

$$
\begin{aligned}
I_{n, 0}(\phi)_{U_{1}}(g) & =\tau\left(H_{0}\right)^{-1} \cdot \int_{\left[U_{1}\right]} \int_{\left[H_{0}\right]} \theta_{n, 0}(\phi)(u g, h) d h d u \\
& =\tau\left(H_{0}\right)^{-1} \cdot \int_{\left[H_{0}\right]} \int_{\left[U_{1}\right]}\left(\sum_{v_{0} \in\left(y_{1}^{*} \otimes V_{0}\right)(F)} \sum_{\alpha \in\left(Y_{n-1}^{\prime *} \otimes V_{0}\right)(F)} \omega_{n, 0}(u g, h) \phi\left(v_{0}, \alpha\right)\right) d u d h .
\end{aligned}
$$


We may break the sum over $v_{0}$ into two parts, corresponding to $v_{0}=0$ and $v_{0} \neq 0$. For $v_{0}=0$, the term in the parenthesis is:

$$
\sum_{\alpha \in\left(Y_{n-1}^{\prime *} \otimes V_{0}\right)(F)} \omega_{n, 0}(g, h) \phi(0, \alpha)=\chi(t) \cdot|t|^{\frac{1}{2} m_{0}} \cdot \theta_{n-1,0}(\phi(0,-))\left(g_{0}, h\right) .
$$

Since this is independent of $u$, the integral over $\left[U_{1}\right]$ disappears and the outer integral over $\left[H_{0}\right]$ gives $I_{n-1,0}(\phi(0,-))$ : this is the desired RHS in the identity of the proposition.

To prove the proposition, it remains to show that the contribution from the term $v_{0} \neq 0$ vanishes. For this, we shall show that the integral

$$
\int_{\left[Z_{1}\right]} \sum_{v_{0} \neq 0} \sum_{\alpha \in\left(Y_{n-1}^{\prime *} \otimes V_{0}\right)(F)} \omega_{n, 0}(z g, h) \phi\left(v_{0}, \alpha\right) d z=0 .
$$

Indeed,

$$
\omega_{n, 0}(z g, h) \phi\left(v_{0}, \alpha\right)=\psi\left(\frac{1}{2} \cdot z \cdot\left(v_{0}, v_{0}\right)\right) \cdot \phi\left(v_{0}, \alpha\right) .
$$

Since $V_{0}$ is anisotropic and $v_{0} \neq 0$, we see that $\left(v_{0}, v_{0}\right) \neq 0$ so that

$$
z \mapsto \psi\left(\frac{1}{2} \cdot z \cdot\left(v_{0}, v_{0}\right)\right)
$$

is a nontrivial character on $\left[Z_{1}\right]$. This implies that the above integral over $\left[Z_{1}\right]$ vanishes, as desired.

7.2. First term identity. In this section, we recall the regularized Siegel-Weil formula in the first term range. The following theorem was shown by Kudla-Rallis [KR5, Moeglin [Mo, Ichino [1, Theorem 3.1] and [12, Theorems 4.1 and 4.2]), Jiang-Soudry [JS, Theorem 2.4] and Yamana [Y2, Proposition 5.8]:

Theorem 7.3. (i) Suppose that $m+m^{\prime}=2 \cdot d(n)$ with $m>m^{\prime}>0$ and $m^{\prime}=m_{0}+2 r^{\prime}$ (here we allow $\left.r^{\prime}=0\right)$. Then for $\phi \in \mathcal{S}\left(Y_{n}^{*} \otimes V_{r}\right)(\mathbb{A})$, we have:

$$
A_{-1}^{n, r}(\phi)=\kappa_{r, r^{\prime}} \cdot B_{*}^{n, r^{\prime}}\left(\mathrm{Ik}^{n, r}\left(\pi_{K_{H_{r}}} \phi\right)\right),
$$

where $\kappa_{r, r^{\prime}}$ is an explicit nonzero constant defined in (3.6), and

$$
*=\left\{\begin{array}{l}
0, \text { if } H_{r^{\prime}}=\mathrm{O}_{1,1} \text { or } r^{\prime}=0 \\
-1 \text { otherwise }
\end{array}\right.
$$

(ii) In the boundary case, where $m=m^{\prime}=d(n)$ (and $r>0$ ), one has:

$$
A_{0}^{n, r}(\phi)=2 \cdot B_{-1}^{n, r}(\phi)
$$

If $H_{r}=\mathrm{O}_{1,1}$ (so that $G_{n}=\mathrm{Sp}_{2}$ ), however, both sides of the above identity are 0 , in which case one has

$$
A_{1}^{n, r}(\phi)=2 \cdot \frac{\tau\left(H_{r}\right)}{[E: F]} \cdot B_{0}^{n, r}(\phi)=B_{0}^{n, r}(\phi)
$$

It should be mentioned that Theorem 7.3 (i) may not seem so natural, since the pair $\left(n, r^{\prime}\right)$ is in the first term range, but we are considering $\phi \in \mathcal{S}\left(Y_{n}^{*} \otimes V_{r}\right)(\mathbb{A})$ which is the Weil representation in the second term range. When $r^{\prime}=0$ in Theorem 7.3(i), Theorem 7.1 gives an alternative, more natural statement. When $r^{\prime}>0$, such a more natural statement, similar to Theorem 7.1 and Theorem 7.3(ii), has been given by Yamana in [Y2, Theorem 2.2]: 
Theorem 7.4. Suppose that $0<m^{\prime}=m_{0}+2 r^{\prime}<d(n)$ with $r^{\prime}>0$. Then for $\phi \in \mathcal{S}\left(Y_{n}^{*} \otimes V_{r^{\prime}}\right)(\mathbb{A})$, $E\left(s, \Phi^{n, r^{\prime}}(\phi)\right)$ is holomorphic at $s=s_{m^{\prime}, n}<0$, and

$$
A_{0}^{n, r^{\prime}}(\phi)=2 \cdot B_{-1}^{n, r^{\prime}}(\phi) .
$$

If $H_{r^{\prime}}=\mathrm{O}_{1,1}$, however, both sides of the identity are zero, in which case we have:

$$
A_{1}^{n, r^{\prime}}(\phi)=2 \cdot \frac{\tau\left(H_{r^{\prime}}\right)}{[E: F]} \cdot B_{0}^{n, r^{\prime}}(\phi)=B_{0}^{n, r^{\prime}}(\phi) .
$$

In particular, Theorem 7.1 and Theorem 7.4 complete the theory of the (regularized) Siegel-Weil formula when $m \leq d(n)$.

\section{The Second Term Identity}

Now we come to the heart of this paper: the derivation of the first and second term identities in the second term range. The goal is to relate the automorphic forms $B_{-1}^{n, r}(\phi)$ and $B_{-2}^{n, r}(\phi)$ with $A_{0}^{n, r}(\phi)$ and $A_{-1}^{n, r}(\phi)$.

8.1. Main result. The following is the main result of this paper.

Theorem 8.1. Assume the pair $(n, r)$ is in the second term range.

(i) (First term identity) For $\phi \in \mathcal{S}\left(Y_{n}^{*} \otimes V_{r}\right)(\mathbb{A})$, one has

$$
A_{-1}^{n, r}(\phi)=B_{-2}^{n, r}(\phi) \text {. }
$$

(ii) (Second term identity) For $\phi \in \mathcal{S}\left(Y_{n}^{*} \otimes V_{r}\right)(\mathbb{A})$, one has

$$
A_{0}^{n, r}(\phi)=B_{-1}^{n, r}(\phi)-\kappa_{r, r^{\prime}} \cdot\left\{B_{0}^{n, r^{\prime}}\left(\mathrm{Ik}^{n, r}\left(\pi_{K_{H_{r}}} \phi\right)\right)\right\} \quad \bmod \operatorname{Im} A_{-1}^{n, r},
$$

where $\kappa_{r, r^{\prime}}$ is a nonzero explicit constant defined in (3.6) and $V_{r^{\prime}}$ is the complementary space of $V_{r}$ (relative to $\left.W_{n}\right)$, so that $m_{0}+r+r^{\prime}=d(n)$. Moreover, the term in $\{\ldots .$.$\} on the RHS is interpreted$ as 0 if $r^{\prime}=0$ or $H_{r^{\prime}}=\mathrm{O}_{1,1}$.

8.2. Strategy. Before plunging into the proof of the theorem, let us give a sketch of the main idea. The strategy of the proof has already been used in GT to prove a weak form of the theorem in the case when $\epsilon_{0}=-1$. It is based on induction on the quantity

$$
\mathcal{N}=m-d(n) .
$$

Note that

$$
\begin{aligned}
& \mathcal{N}<0 \Longleftrightarrow \text { first term range; } \\
& \mathcal{N}=0 \Longleftrightarrow \text { boundary case; } \\
& \mathcal{N}>0 \Longleftrightarrow \text { second term range. }
\end{aligned}
$$

Let us illustrate how one starts the induction argument by going from the theorem in the boundary case $(\mathcal{N}=0)$ to the first case in the second term range $(\mathcal{N}=1)$. Thus suppose we are dealing with the Weil representation of $G\left(W_{n+1}\right) \times H\left(V_{r}\right)$ with $m=d(n+1)$. Then we are in the boundary case, and for $\phi \in \mathcal{S}\left(Y_{n+1}^{*} \otimes V_{r}\right)(\mathbb{A})$ we have the first term identity supplied by Theorem 7.3 (ii):

$$
A_{0}^{n+1, r}(\phi)=2 \cdot B_{-1}^{n+1, r}(\phi) .
$$

We may take the constant term of both sides with respect to the maximal parabolic $Q^{n+1}\left(Y_{1}\right)=L^{n+1}\left(Y_{1}\right)$. $U^{n+1}\left(Y_{1}\right)$ of $G_{n+1}$, which gives

$$
A_{0}^{n+1, r}(\phi)_{U^{n+1}\left(Y_{1}\right)}=2 \cdot B_{-1}^{n+1, r}(\phi)_{U^{n+1}\left(Y_{1}\right)},
$$


which is an identity of automorphic forms on $L\left(Y_{1}\right)=\mathrm{GL}\left(Y_{1}\right) \times G\left(W_{n}\right)$, where $W_{n}=Y_{n}^{\prime} \oplus Y_{n}^{\prime *}$. (Note that the superscript ${ }^{n+1}$ in the groups $Q^{n+1}\left(Y_{1}\right)$ etc indicates the rank of the ambient group $G_{n+1}$.)

Now note that both sides of this last equation are constant terms of the Laurent coefficients of some Eisenstein series. Thus we may compute them by first computing the constant term of the relevant Eisenstein series, followed by extracting the relevant Laurent coefficients. The constant terms along $U^{n+1}\left(Y_{1}\right)$ of Eisenstein series on $G_{n+1}$ are simply the sum of Eisenstein series on $L\left(Y_{1}\right)$, as given in Lemma 8.5 below. We can compare terms on both sides with the same $\operatorname{GL}\left(Y_{1}\right)$ part, and thus obtain an identity of Eisenstein series on $G\left(W_{n}\right)$. It remains to identify these Eisenstein series and the sections where they are evaluated with the desired objects coming from the Weil representation of $G_{n} \times H_{r}$. The theorem will then follow by extracting the relevant Laurent coefficients.

8.3. Constant term of Eisenstein series. We now describe the constant term of the relevant Eisenstein series on $G_{n}$ along the unipotent radical of the parabolic whose Levi part is isomorphic to $G L_{1} \times G_{n-1}$. To be specific, we shall express elements of $G_{n}$ as matrices using the ordered basis $\left\{y_{1}, \ldots, y_{r}, y_{r}^{*}, \ldots, y_{1}^{*}\right\}$ of $W_{n}$. We let

$$
Y_{1}=\left\langle y_{1}\right\rangle
$$

and

$$
Q\left(Y_{1}\right)=L\left(Y_{1}\right) \cdot U\left(Y_{1}\right)
$$

the parabolic subgroup that fixes $Y_{1}$ with Levi part

$$
L\left(Y_{1}\right)=G L\left(Y_{1}\right) \times G\left(W_{n-1}\right) \cong \mathrm{GL}_{1} \times G_{n-1} .
$$

While the computation of the constant terms of Eisenstein series is standard, it is necessary to introduce some notation to state the results.

Let $f_{s}$ be a standard section of

$$
I_{r}^{n}(s, \chi):=\operatorname{Ind}_{Q\left(Y_{r}\right)(\mathbb{A})}^{G_{n}(\mathbb{A})}(\chi \circ \operatorname{det}) \cdot|\operatorname{det}|^{s} \otimes \Theta_{n-r, 0}\left(V_{0}\right) .
$$

Since the elements of the inducing data $(\chi \circ \operatorname{det}) \cdot|\operatorname{det}|^{s} \otimes \Theta_{n-r, 0}\left(V_{0}\right)$ are automorphic forms on $L\left(Y_{r}\right)$, via evaluation at the identity element of $L\left(Y_{r}\right)$, we may regard $f_{s}$ as a $\mathbb{C}$-valued function on $U\left(Y_{r}\right)(\mathbb{A}) L\left(Y_{r}\right)(F) \backslash G_{n}(\mathbb{A})$.

Now let $E^{n, r}\left(s, f_{s}\right)$ be the associated Eisenstein series, i.e.

$$
E^{n, r}\left(s, f_{s}\right)(g):=\sum_{\gamma \in Q\left(Y_{r}\right)(F) \backslash G_{n}(F)} f_{s}(\gamma g)
$$

for $g \in G_{n}(\mathbb{A})$ and $\operatorname{Re}(s) \gg 0$. Assume that $1 \leq r<n$. Then in computing the constant term $E^{n, r}(s, f)_{U\left(Y_{1}\right)}$, we first need to enumerate the double coset space $Q\left(Y_{r}\right) \backslash G_{n} / Q\left(Y_{1}\right)$. In this case, one knows that the double coset representatives can be chosen to be the elements $1, w^{+}$and $w^{-}$, where

$$
w^{+}=\left(\begin{array}{cc}
J_{r+1} & 0 \\
0 & J_{r+1}
\end{array}\right)
$$

with

$$
J_{r+1}=\left(\begin{array}{cccc}
0 & 0 & 1 & 0 \\
0 & I_{r-1} & 0 & 0 \\
1 & 0 & 0 & 0 \\
0 & 0 & 0 & I_{n-r-1}
\end{array}\right)
$$

and

$$
w^{-}=\left(\begin{array}{ccc}
0 & 0 & 1 \\
0 & I_{2 n-2} & 0 \\
-\epsilon & 0 & 0
\end{array}\right) .
$$


Note that there is a distinguished set of coset representatives, given by Weyl group elements of minimal length, and the elements $w^{+}$and $w^{-}$given above are not equal to these distinguished elements. However, they are more convenient for our purposes; for example they have the convenient property that they are essentially their own inverses.

Associated to the Weyl group element $w=w^{+}$or $w^{-}$is the standard intertwining operator $M(w, s)$ :

$$
M(w, s)(f)(g)=\int_{\left(U\left(Y_{1}\right)(F) \cap w Q\left(Y_{r}\right)(F) w^{-1}\right) \backslash U\left(Y_{1}\right)(\mathbb{A})} f_{s}\left(w^{-1} u g\right) d u .
$$

To study these intertwining operators in greater depth, let us write

$$
U\left(Y_{1}\right) \cap w Q\left(Y_{r}\right) w^{-1}=\left(U\left(Y_{1}\right) \cap w U\left(Y_{r}\right) w^{-1}\right) \cdot\left(U\left(Y_{1}\right) \cap w L\left(Y_{r}\right) w^{-1}\right),
$$

and note that

$$
\begin{aligned}
M(w, s)(f)(g) & =\int_{\left(U\left(Y_{1}\right)(\mathbb{A}) \cap w Q\left(Y_{r}\right)(\mathbb{A}) w^{-1}\right) \backslash U\left(Y_{1}\right)(\mathbb{A})} \int_{\left[U\left(Y_{1}\right) \cap w L\left(Y_{r}\right) w^{-1}\right]} f_{s}\left(w^{-1} \cdot v \cdot u \cdot g\right) d v d u \\
& =\int_{U\left(Y_{1}\right)^{w}(\mathbb{A})}\left(\int_{\left[L\left(Y_{r}\right) \cap w^{-1} U\left(Y_{1}\right) w\right]} f_{s}\left(v \cdot w^{-1} \cdot u \cdot g\right) d v\right) d u
\end{aligned}
$$

where we have set

$$
U\left(Y_{1}\right)^{w}:=U\left(Y_{1}\right) \cap w Q\left(Y_{r}\right) w^{-1} \backslash U\left(Y_{1}\right) .
$$

Now we can make (8.3) explicit as follows:

- When $w=w^{+}, L\left(Y_{r}\right) \cap w^{-1} U\left(Y_{1}\right) w$ is the unipotent radical of the maximal parabolic $Q_{1}^{n-r}$ of $G_{n-r} \subset$ $L_{r}$ whose Levi is isomorphic to $\mathrm{GL}_{1} \times G_{n-r-1}$. (To be more precise, $Q_{1}^{n-r}$ is the parabolic that fixed the space $\left\langle y_{r+1}\right\rangle$. For the notation $Q_{1}^{n-r}$, the superscript indicates the rank of the ambient group and the subscript indicates the rank of the GL-part of the Levi.) Thus the inner integral is the constant term of $f_{s}$ along this unipotent radical. We denote this constant term by $R_{Q_{1}}^{n-r}\left(f_{s}\right)$, namely

$$
R_{Q_{1}}^{n-r}\left(f_{s}\right)(g)=\int_{\left[L\left(Y_{r}\right) \cap w^{-1} U\left(Y_{1}\right) w\right]} f_{s}(v g) d v
$$

for $g \in G_{n}(\mathbb{A})$. One can see that

$$
R_{Q_{1}}^{n-r}\left(f_{s}\right) \in \operatorname{Ind}_{Q_{r, 1}^{n}(\mathbb{A})}^{G_{n}(\mathbb{A})}(\chi \circ \operatorname{det})|\operatorname{det}|^{s} \otimes|-|^{-\left(n-r-\frac{1}{2}+\frac{\epsilon_{0}}{2}\right)} \otimes R_{Q_{1}}^{n-r} \Theta_{n-r, 0}\left(V_{0}\right)
$$

where $Q_{r, 1}^{n}$ is the non-maximal parabolic of $G_{n}$ with Levi factor $\operatorname{GL}\left(Y_{r}\right) \times G L_{1} \times G_{n-r-1}$, and $R_{Q_{1}}^{n-r} \Theta_{n-r, 0}\left(V_{0}\right)$ is the space generated by the constant terms of the automorphic forms in $\Theta_{n-r, 0}\left(V_{0}\right)$ along the unipotent radical of $Q_{1}^{n-r}$. By Proposition 7.2 , we have

$$
R_{Q_{1}}^{n-r} \Theta_{n-r, 0}\left(V_{0}\right)=\chi|-|^{\frac{1}{2} m_{0}} \otimes \Theta_{n-r-1,0}\left(V_{0}\right),
$$

so that

$$
R_{Q_{1}}^{n-r}\left(f_{s}\right) \in \operatorname{Ind}_{Q_{r, 1}^{n}(\mathbb{A})}^{G_{n}(\mathbb{A})}\left((\chi \circ \operatorname{det})|\operatorname{det}|^{s} \otimes \chi|-|^{\frac{1}{2} m_{0}-\left(n-r-\frac{1}{2}+\frac{\epsilon_{0}}{2}\right)} \otimes \Theta_{n-r-1,0}\left(V_{0}\right)\right) .
$$

Thus

$$
M\left(w^{+}, s\right)(f)(g)=\int_{U_{1}^{w+}(\mathbb{A})} R_{Q_{1}}^{n-r}\left(f_{s}\right)\left(w^{+} u g\right) d u .
$$

On restricting $M\left(w^{+}, s\right) f$ to the subgroup $L\left(Y_{1}\right)=\mathrm{GL}\left(Y_{1}\right) \times G_{n-1}$, we have:

$$
\left.M\left(w^{+}, s\right)(f)\right|_{L\left(Y_{1}\right)} \in \chi \cdot|-|^{m / 2} \otimes I_{r}^{n-1}(s, \chi) .
$$


- When $w=w^{-}, L\left(Y_{r}\right) \cap w^{-1} U\left(Y_{1}\right) w$ is the unipotent radical of a maximal parabolic subgroup of $\operatorname{GL}\left(Y_{r}\right) \subset L\left(Y_{r}\right)=G L\left(Y_{r}\right) \times G_{n-r}$. Since the function $f$ transforms under GL $\left(Y_{r}\right)$ by the character $(\chi \circ \operatorname{det}) \cdot|\operatorname{det}|^{s}$, we see that the integrand of this inner integral of (8.3) is independent of $v$ and so the inner integral simply disappears and gives

$$
M\left(w^{-}, s\right)(f)(g)=\int_{U_{1}^{w^{-}}(\mathbb{A})} f_{s}\left(w^{-} u g\right) d u .
$$

One has:

$$
\left.M\left(w^{-}, s\right)(f)\right|_{L\left(Y_{1}\right)} \in \chi \cdot|-|^{-s+n-\frac{r-\epsilon_{0}}{2}} \otimes I_{r-1}^{n-1}\left(s-\frac{1}{2}, \chi\right) .
$$

With the above notation, we can now state the lemma which computes the constant term $E^{n, r}(s, f)_{U\left(Y_{1}\right)}$ as follows.

Lemma 8.5. Let $f_{s}$ be a standard section of $I_{r}^{n}(s, \chi)=\operatorname{Ind}_{Q\left(Y_{r}\right)}^{G_{n}}(\chi \circ \operatorname{det})|\operatorname{det}|^{s} \otimes \Theta_{n-r, 0}\left(V_{0}\right)$ and let $E^{n, r}(s, f)$ be the associated Eisenstein series.

(i) If $1 \leq r<n$,

$$
\begin{aligned}
E^{n, r}(s, f)_{U\left(Y_{1}\right)} & =\chi|-|^{s+n-\frac{r-\epsilon_{0}}{2}} \cdot E^{n-1, r-1}\left(s+\frac{1}{2},\left.f\right|_{G_{n-1}}\right) \\
& +\chi|-|^{m / 2} \cdot E^{n-1, r}\left(s,\left.M\left(w^{+}, s\right)(f)\right|_{G_{n-1}}\right) \\
& +\chi|-|^{-s+n-\frac{r-\epsilon_{0}}{2}} \cdot E^{n-1, r-1}\left(s-\frac{1}{2},\left.M\left(w^{-}, s\right)(f)\right|_{G_{n-1}}\right) .
\end{aligned}
$$

(ii) If $r=n$,

$$
\begin{aligned}
E^{n, r}(s, f)_{U\left(Y_{1}\right)} & =\chi|-|^{s+\frac{d(n)}{2}} \cdot E^{n-1, n-1}\left(s+\frac{1}{2},\left.f\right|_{G_{n-1}}\right) \\
& +\chi|-|^{-s+\frac{d(n)}{2}} \cdot E^{n-1, n-1}\left(s-\frac{1}{2},\left.M\left(w^{-}, s\right)(f)\right|_{G_{n-1}}\right) .
\end{aligned}
$$

By applying the same type of computation to the auxiliary Eisenstein series $E_{H_{r}}$, we have

Lemma 8.6. Let $f_{s}$ be a standard section in $I_{H_{r}}(s)=\operatorname{Ind}_{P\left(X_{r}\right)(\mathbb{A})}^{H_{r}(\mathbb{A})}\left|\operatorname{det}_{X_{r}}\right|^{s} \otimes \mathbf{1}_{H_{0}}$, and let $E_{H_{r}}(s, f)$ be associated Eisenstein series. Let $P\left(X_{1}\right)=M\left(X_{1}\right) \cdot N\left(X_{1}\right)$ be the parabolic subgroup that preserves $X_{1}=\left\langle x_{1}\right\rangle$, where $M\left(X_{1}\right)=G L\left(X_{1}\right) \times H_{r-1}$ is the Levi part and $N\left(X_{1}\right)$ is the unipotent radical. Then

where

$$
\begin{aligned}
E_{H_{r}}(s, f)_{N\left(X_{1}\right)} & =\chi|-|^{s+\frac{m-r+\epsilon_{0}}{2}} \cdot E_{H_{r-1}}\left(s+\frac{1}{2},\left.f\right|_{H_{r-1}}\right) \\
& +\chi|-|^{-s+\frac{m-r+\epsilon_{0}}{2}} \cdot E_{H_{r-1}}\left(s-\frac{1}{2},\left.M\left(w^{-}, s\right)(s)(f)\right|_{H_{r-1}}\right)
\end{aligned}
$$

$$
w^{-}=\left(\begin{array}{ccc}
0 & 0 & 1 \\
0 & I_{m-2} & 0 \\
\epsilon & 0 & 0
\end{array}\right) \text {. }
$$

8.4. Proof of Lemma 3.4(i). We can now give a proof of Lemma 3.4(i) using Lemma 8.6 and induction on $r$.

Let us choose $f_{s}=f_{s}^{0}$ to be the spherical section in Lemma 8.6, and write $E_{H_{r}}\left(s, f^{0}\right)=E_{H_{r}}(s)$. If $H_{r}=\mathrm{O}_{1,1}$ (i.e. $m=2, r=1$ and $\epsilon_{0}=1$ ), it is easy to see directly that $E_{H_{1}}(s)$ and $c_{1}(s)$ are entire, with $\kappa_{r}=2$ and $c_{1}(s)=1$, so that Lemma 3.4(i) holds in this case. Thus, we assume that $H_{r} \neq \mathrm{O}_{1,1}$ henceforth, so that $E_{H_{r}}(s)$ has a pole of order 1 at $s=\rho_{H_{r}}$. 
Taking the constant term $E_{H_{r}}(s)_{N\left(X_{1}\right)}$ along $N\left(X_{1}\right)$, we observe that on the RHS of the identity in Lemma 8.6, the first term is holomorphic at $s=\rho_{H_{r}}$. Thus, we have

$$
-1=\operatorname{ord}_{s=\rho_{H_{r}}} E_{H_{r}}(s)_{N\left(X_{1}\right)}=\operatorname{ord}_{s=\rho_{H_{r}}} E_{H_{r-1}}\left(s-\frac{1}{2},\left.M\left(w^{-}, s\right)(s)\left(f^{0}\right)\right|_{H_{r-1}}\right) .
$$

By the analog of KR3, Lemma 1.2.2], one has

$$
M_{H_{r-1}}\left(s-\frac{1}{2}\right)\left(\left.\left(M\left(w^{-}, s\right) f^{0}\right)\right|_{H_{r-1}}\right)=\left.M_{H_{r}}(s) f^{0}\right|_{H_{r-1}} .
$$

Thus

$$
\begin{aligned}
E_{H_{r-1}}\left(s-\frac{1}{2},\left.\left(M\left(w^{-}, s\right) f^{0}\right)\right|_{H_{r-1}}\right) & =E_{H_{r-1}}\left(-s+\frac{1}{2}, M_{H_{r-1}}\left(s-\frac{1}{2}\right)\left(\left.\left(M\left(w^{-}, s\right) f^{0}\right)\right|_{H_{r-1}}\right)\right. \\
& =E_{H_{r-1}}\left(-s+\frac{1}{2},\left.M_{H_{r}}(s) f^{0}\right|_{H_{r-1}}\right) \\
& =c_{r}(s) \cdot E_{H_{r-1}}\left(-s+\frac{1}{2}, f_{r-1}^{0}\right) \\
& =\frac{c_{r}(s)}{c_{r-1}(s-1 / 2)} \cdot E_{H_{r-1}}\left(s-\frac{1}{2}\right)
\end{aligned}
$$

where for the first equation we used the functional equation for $E_{H_{r-1}}$ and also $f_{r-1}^{0}$ denotes the spherical section corresponding to the group $H_{r-1}$.

When $r=1$ (recalling that $H_{1} \neq \mathrm{O}_{1,1}$ here), the last expression is simply $c_{1}(s)$ and so we see that $c_{1}(s)$ has a pole of order 1 at $s=\rho_{H_{1}}$ and

$$
\kappa_{1}=\operatorname{Res}_{s=\rho_{H_{1}}} E_{H_{1}}(s)=\operatorname{Res}_{s=\rho_{H_{1}}} c_{1}(s),
$$

as desired. This completes the verification of the base case of the induction.

For $r>1$, we see that

$$
\operatorname{ord}_{s=\rho_{H_{r}}} E_{H_{r}}(s)=\operatorname{ord}_{s=\rho_{H_{r}}} \frac{c_{r}(s)}{c_{r-1}(s-1 / 2)} \cdot E_{H_{r-1}}\left(s-\frac{1}{2}\right) .
$$

On noting that $\rho_{H_{r}}-1 / 2=\rho_{H_{r-1}}$, we conclude that

$$
\operatorname{ord}_{s=\rho_{H_{r}}}\left(\frac{E_{H_{r}}(s)}{c_{r}(s)}\right)=\operatorname{ord}_{s=\rho_{H_{r-1}}}\left(\frac{E_{H_{r-1}}(s)}{c_{r-1}(s)}\right) .
$$

The result thus follows by induction on $r$. This completes the proof of Lemma 3.4(i).

\section{Proof of Theorem 8.1; Base Step}

In this section, we shall begin our proof of Theorem 8.1 by establishing the base case, which goes from $\mathcal{N}=0$ to $\mathcal{N}=1$.

9.1. Base step of induction. Choose once and for all $\phi_{1} \in \mathcal{S}\left(Y_{1}^{*} \otimes V_{r}\right)(\mathbb{A})$ satisfying:

- $\phi_{1}(0)=1$

- $\phi_{1}$ is $K_{H_{r}}$-invariant, so that $\pi_{K_{H_{r}}} \phi_{1}=\phi_{1}$.

Let $Y_{n}^{\prime}=\left\langle y_{2}, \ldots, y_{n+1}\right\rangle$ so that $Y_{n}^{\prime *}=\left\langle y_{2}^{*}, \ldots, y_{n+1}^{*}\right\rangle$. For any $\phi \in \mathcal{S}\left(Y_{n}^{\prime *} \otimes V_{r}\right)(\mathbb{A})$, we set

$$
\tilde{\phi}:=\phi_{1} \otimes \phi \in \mathcal{S}\left(Y_{n+1}^{*} \otimes V_{r}\right)(\mathbb{A}) \text {. }
$$

Then

$$
\pi_{K_{H_{r}}}(\tilde{\phi})=\phi_{1} \otimes \pi_{K_{H_{r}}} \phi
$$

Note that the group $G_{n}$ acts trivially on $\phi_{1}$, i.e. for $g \in G_{n}(\mathbb{A}), \omega_{n+1, r}(g) \tilde{\phi}=\phi_{1} \otimes \omega_{n, r}(g) \phi$. 
We consider the boundary case: $\left(W_{n+1}, V_{r}\right)$ with $m=d(n+1)$. Then from the first term identity for the boundary case, we have

$$
A_{0}^{n+1, r}(\tilde{\phi})=2 \cdot B_{-1}^{n+1, r}(\tilde{\phi}) .
$$

We compute the constant term along $U\left(Y_{1}\right)$ to get:

$$
A_{0}^{n+1, r}(\tilde{\phi})_{U\left(Y_{1}\right)}=2 \cdot B_{-1}^{n+1, r}(\tilde{\phi})_{U\left(Y_{1}\right)} .
$$

Now we may appeal to Lemma 8.5 to calculate both sides. We explicate the results below.

9.2. The A-side. Let us start with the LHS of this identity (9.1), namely "the $A$-side". We have:

$$
A_{0}^{n+1, r}(\tilde{\phi})_{U\left(Y_{1}\right)}=\operatorname{Val}_{s=0} E^{n+1, n+1}\left(s, \Phi^{n+1, r}(\tilde{\phi})\right)_{U\left(Y_{1}\right)} .
$$

On the other hand, Lemma 8.5 (ii) says that, as an automorphic form on $L\left(Y_{1}\right)=\operatorname{GL}\left(Y_{1}\right) \times G\left(W_{n}\right)$,

$$
\begin{aligned}
E^{n+1, n+1}\left(s, \Phi^{n+1, r}(\tilde{\phi})\right)_{U\left(Y_{1}\right)} & =\chi|-|^{s+\frac{d(n+1)}{2}} \cdot E^{n, n}\left(s+\frac{1}{2},\left.\Phi^{n+1, r}(\tilde{\phi})\right|_{G_{n}}\right) \\
& +\chi|-|^{-s+\frac{d(n+1)}{2}} \cdot E^{n, n}\left(s-\frac{1}{2},\left.M\left(w^{-}, s\right)\left(\Phi^{n+1, r}(\tilde{\phi})\right)\right|_{G_{n}}\right) .
\end{aligned}
$$

Observe that at $s=0, \mathrm{GL}\left(Y_{1}\right)$ acts by the same character $\chi \cdot|-|^{\frac{d(n+1)}{2}}$ for both terms of RHS of (9.2).

Now note that for $g \in G_{n}(\mathbb{A})$,

$$
\Phi^{n+1, r}(\tilde{\phi})(g)=\omega_{n+1, r}(g) \tilde{\phi}(0)=\phi_{1}(0) \cdot \omega_{n, r}(g) \phi(0)=\Phi^{n, r}(\phi)(g),
$$

so that the first term on the RHS of (9.2) is simply

$$
E^{n, n}\left(s+\frac{1}{2}, \Phi^{n, r}(\phi)\right)
$$

as a function on $G_{n}$. Thus, taking the value of the first term on the RHS at $s=0$, we get

$$
A_{0}^{n, r}(\phi) \text {. }
$$

Next let us consider the second term on the RHS of (9.2). By applying the functional equation, we obtain

$$
E^{n, n}\left(s-\frac{1}{2},\left.M\left(w^{-}, s\right)\left(\Phi^{n+1, r}(\tilde{\phi})\right)\right|_{G_{n}}\right)=E^{n, n}\left(\frac{1}{2}-s, M_{n}\left(s-\frac{1}{2}, \chi\right)\left(\left.M\left(w^{-}, s\right)\left(\Phi^{n+1, r}(\tilde{\phi})\right)\right|_{G_{n}}\right)\right),
$$

where recall $M_{n}(s, \chi)$ is the intertwining operator for the Siegel principal series. Moreover, it was shown in [KR3, Lemma 1.2.2] that

$$
M_{n}\left(s-\frac{1}{2}, \chi\right)\left(\left.M\left(w^{-}, s\right)\left(\Phi^{n+1, r}(\tilde{\phi})\right)\right|_{G_{n}}\right)=\left.M_{n+1}(s, \chi)\left(\Phi^{n+1, r}(\tilde{\phi})\right)\right|_{G_{n}} .
$$

Thus the second term on the RHS of (9.2) is equal to:

$$
E^{n, n}\left(\frac{1}{2}-s,\left.M_{n+1}(s)\left(\Phi^{n+1, r}(\tilde{\phi})\right)\right|_{G_{n}}\right) .
$$

Moreover, the intertwining operator $M_{n+1}(s)$ is holomorphic at $s=0$ and

$$
M_{n+1}(0)\left(\Phi^{n+1, r}(\tilde{\phi})\right)=\Phi^{n+1, r}(\tilde{\phi})
$$

by Lemma 6.3(ii). Thus, on taking the value at $s=0$, the second term of the RHS of (9.2) gives

$$
A_{0}^{n, r}(\phi)-E_{-1, s_{m, n}}^{n, n}\left(\left.M_{n+1}^{\prime}(0, \chi)\left(\Phi^{n+1, r}(\tilde{\phi})\right)\right|_{G_{n}}\right),
$$

where we note that $s_{m, n}=1 / 2$ here. By Lemma 6.3(iii) we know the derivative $M_{n+1}^{\prime}(0, \chi)$ preserves each irreducible submodule of $I_{n+1}^{n+1}(0, \chi)$, and hence

$$
M_{n+1}^{\prime}(0, \chi)\left(\Phi^{n+1, r}(\tilde{\phi})\right)=\Phi^{n+1, r}(\varphi) \quad \text { for some } \varphi \in \mathcal{S}\left(Y_{n+1}^{*} \otimes V_{r}\right)(\mathbb{A}),
$$


so that

So (9.4) is written as

$$
\left.M_{n+1}^{\prime}(0, \chi)\left(\Phi^{n+1, r}(\tilde{\phi})\right)\right|_{G_{n}}=\Phi^{n, r}\left(\left.\varphi\right|_{Y_{n}^{*} \otimes V_{r}}\right) \in \operatorname{Im} \Phi^{n, r} .
$$

$$
A_{0}^{n, r}(\phi)-A_{-1}^{n, r}\left(\left.\varphi\right|_{Y_{n}^{*} \otimes V_{r}}\right) .
$$

From this together with (9.3), we conclude

$$
A_{0}^{n+1, r}(\tilde{\phi})_{U\left(Y_{1}\right)}=2 \cdot A_{0}^{n, r}(\phi) \bmod \operatorname{Im}\left(A_{-1}^{n, r}\right) .
$$

9.3. The B-side. Now we consider the RHS ("the B-side") of (9.1), which is decidedly more complicated. Lemma 8.5 (i) implies that $B_{-1}^{n+1, r}(\tilde{\phi})_{U\left(Y_{1}\right)}$ is the residue at

$$
s=\rho_{H_{r}}=\frac{m-r-\epsilon_{0}}{2}=\frac{d(n+1)-r-\epsilon_{0}}{2}=\frac{n+1-r}{2}
$$

of the function

$$
\begin{aligned}
& \chi|-|^{s+n+1-\frac{r-\epsilon_{0}}{2}} \cdot E^{n, r-1}\left(s+\frac{1}{2},\left.f^{n+1, r}\left(s, \pi_{K_{H_{r}}} \tilde{\phi}\right)\right|_{G_{n}}\right) \\
+ & \chi|-|^{m / 2} \cdot E^{n, r}\left(s,\left.M\left(w^{+}, s\right) f^{n+1, r}\left(s, \pi_{K_{H_{r}}} \tilde{\phi}\right)\right|_{G_{n}}\right) \\
+ & \chi|-|^{-s+n+1-\frac{r-\epsilon_{0}}{2}} \cdot E^{n, r-1}\left(s-\frac{1}{2},\left.M\left(w^{-}, s\right) f^{n+1, r}\left(s, \pi_{K_{H_{r}}} \tilde{\phi}\right)\right|_{G_{n}}\right) .
\end{aligned}
$$

The first thing we should mention is that at $s=\rho_{H_{r}}, \operatorname{GL}\left(Y_{1}\right)$ acts by the character $\chi|-| \frac{1}{2} m+(n+1-r)$ in the first term whereas it acts by $\chi|-|^{d(n+1) / 2}=\chi|-|^{m / 2}$ in the other two terms. This implies that the first term does not have a residue at $s=\rho_{H_{r}}$ because both terms on the A-side of (9.1) has GL( $\left.Y_{1}\right)$ acting via $\chi \cdot|-|^{m / 2}$. Alternatively one can directly prove that the first term of (9.6) does not have a residue as follows:

Lemma 9.7. The Eisenstein series $E^{n, r-1}\left(s+\frac{1}{2},\left.f^{n+1, r}\left(s, \pi_{K_{H_{r}}} \tilde{\phi}\right)\right|_{G_{n}}\right)$ is holomorphic at $s=\rho_{H_{r}}$.

Proof. Since $m=d(n+1)$, the complementary space $V_{r^{\prime}}$ of $V_{r}$ relative to $W_{n}$ has $r^{\prime}=r-1$. By Proposition 4.4, we see that

$$
\left.f^{n+1, r}\left(s, \pi_{K_{H_{r}}} \tilde{\phi}\right)\right|_{G_{n}}=\alpha_{r} \cdot Z_{1}\left(-s-\rho_{H_{r}}, \phi_{1}\right) \cdot f^{n, r-1}\left(s+\frac{1}{2}, \mathrm{Ik}^{n, r}\left(\pi_{K_{H_{r}}} \phi\right)\right) .
$$

Thus,

$E^{n, r-1}\left(s+\frac{1}{2},\left.f^{n+1, r}\left(s, \pi_{K_{H_{r}}} \tilde{\phi}\right)\right|_{G_{n}}\right)=\alpha_{r} \cdot Z_{1}\left(-s-\rho_{H_{r}}, \phi_{1}\right) \cdot E^{n, r-1}\left(s+\frac{1}{2}, f^{n, r-1}\left(s+\frac{1}{2}, \operatorname{Ik}^{n, r}\left(\pi_{K_{H_{r}}} \phi\right)\right)\right)$.

At $s=\rho_{H_{r}}, Z_{1}\left(-s-\rho_{H_{r}}, \phi_{1}\right)$ is holomorphic, since one is considering a Tate zeta integral at $-2 \rho_{H_{r}}=$ $-(n+1-r) \leq-1$. On the other hand, since $\operatorname{Ik}^{n, r}$ is $H_{r-1}(\mathbb{A})$-equivariant, $\operatorname{Ik}^{n, r}\left(\pi_{K_{H_{r}}} \phi\right)$ is $K_{H_{r-1}}{ }^{-}$ invariant. Hence,

$$
\begin{aligned}
& E^{n, r-1}\left(s+\frac{1}{2}, f^{n, r-1}\left(s+\frac{1}{2}, \mathrm{Ik}^{n, r}\left(\pi_{K_{H_{r}}} \phi\right)\right)\right) \\
= & B^{n, r-1}\left(s+\frac{1}{2}, \mathrm{Ik}^{n, r}\left(\pi_{K_{H_{r}}} \phi\right)\right) \\
= & \frac{1}{\tau\left(H_{r-1}\right) \cdot \kappa_{r-1} \cdot P_{n, r-1}\left(s+\frac{1}{2}\right)} \cdot \int_{\left[H_{r-1}\right]} \theta\left(\omega_{n, r-1}(z) \operatorname{Ik}^{n, r}\left(\pi_{K_{H_{r}}} \phi\right)\right)(g, h) \cdot E_{H_{r-1}}\left(s+\frac{1}{2}, h\right) d h .
\end{aligned}
$$

At $s=\rho_{H_{r}}=\rho_{H_{r-1}}+1 / 2$, one may verify (using formulas for $P_{n, r-1}(s)$ in [KR5, I2, JS] ) that $P_{n, r-1}(s+$ $1 / 2)$ and $E_{H_{r-1}}\left(s+\frac{1}{2}, h\right)$ are both holomorphic and nonzero. This proves the lemma. 
Now we examine the remaining two terms corresponding to $w^{+}$and $w^{-}$in turn.

The term for $w^{+}$: We note the following key proposition:

Proposition 9.8.

$$
\left.M\left(w^{+}, s\right) f^{n+1, r}\left(s, \pi_{K_{H_{r}}} \tilde{\phi}\right)\right|_{G_{n}}=f^{n, r}\left(s, \pi_{K_{H_{r}}} \phi\right) .
$$

Proof. By definition, for $g \in G_{n}$,

$$
M\left(w^{+}, s\right) f^{n+1, r}\left(s, \pi_{K_{H_{r}}} \tilde{\phi}\right)(g)=\int_{\left(U_{1}^{n+1}\right)^{w^{+}}(\mathbb{A})} R_{Q_{1}}^{n+1-r}\left(f^{n+1, r}\left(s, \pi_{K_{H}} \tilde{\phi}\right)\right)\left(w^{+} u g\right) d u,
$$

where recall that as defined in (8.4), $R_{Q_{1}}^{n+1-r}$ indicates the "constant term" along the unipotent radical of the parabolic of $G_{n+1-r}$ whose Levi is $G L_{1} \times G_{n-r}$, and

$$
\begin{aligned}
& f^{n+1, r}\left(s, \pi_{K_{H}} \tilde{\phi}\right)(g) \\
= & \int_{\mathrm{GL}\left(X_{r}\right)(\mathbb{A})} I_{n+1-r, 0}\left(\omega_{n+1, r}(g)\left(\mathcal{F}_{n+1, r}\left(\pi_{K_{H}} \tilde{\phi}\right)\left(\beta_{0} \circ a\right)\left(0_{r},-\right)\right) \cdot|\operatorname{det}(a)|^{s+n+1-\rho_{H_{r}}} d a\right.
\end{aligned}
$$

where $0_{r}$ is the zero element in $Y_{r}^{*} \otimes V_{0}$. Thus

$$
\begin{aligned}
& R_{Q_{1}}^{n+1-r}\left(f^{n+1, r}\left(s, \pi_{K_{H}} \tilde{\phi}\right)\right)(g) \\
= & \int_{\mathrm{GL}\left(X_{r}\right)(\mathbb{A})} R_{Q_{1}}^{n+1-r}\left(I_{n+1-r, 0}\left(\omega_{n+1, r}(g)\left(\mathcal{F}_{n+1, r}\left(\pi_{K_{H}} \tilde{\phi}\right)\left(\beta_{0} \circ a\right)(0,-)\right)\right) \cdot|\operatorname{det}(a)|^{s+n+1-\rho_{H_{r}}} d a .\right.
\end{aligned}
$$

Let us set

$$
\Phi_{a}:=\mathcal{F}_{n+1, r}\left(\pi_{K_{H}} \tilde{\phi}\right)\left(\beta_{0} \circ a\right)(0,-) \in \mathcal{S}\left(Y_{n+1-r}^{\prime}{ }^{*} \otimes V_{0}\right)(\mathbb{A}) .
$$

By Proposition 7.2, we have an identity of functions on $G_{n-r}$ :

$$
R_{Q_{1}}^{n+1-r}\left(I_{n+1-r, 0}\left(\Phi_{a}\right)\right)=I_{n-r, 0}\left(\Phi_{a}(0,-)\right)
$$

where

with $Y_{n-r}^{*}=\left\langle y_{r+2}^{*}, \ldots, y_{n+1}^{*}\right\rangle$.

$$
\Phi_{a}(0,-) \in \mathcal{S}\left(Y_{n-r}^{\prime}{ }^{*} \otimes V_{0}\right)(\mathbb{A})
$$

Hence

$$
\begin{aligned}
& M\left(w^{+}, s\right) f^{n+1, r}\left(s, \pi_{K_{H_{r}}} \tilde{\phi}\right)(g) \\
= & \int_{\left(U_{1}^{n+1}\right)^{w^{+}}(\mathbb{A})} \int_{\mathrm{GL}\left(X_{r}\right)(\mathbb{A})} I_{n-r, 0}\left(\omega_{n+1, r}\left(w^{+} u g, a\right) \mathcal{F}_{n+1, r}\left(\pi_{K_{H}} \tilde{\phi}\right)\left(\beta_{0}\right)\left(0_{r+1},-\right)\right) \cdot|\operatorname{det}(a)|^{s-\rho_{H_{r}}} d a d u
\end{aligned}
$$

where $0_{r+1}$ is the zero element in $Y_{r+1}^{*} \otimes V_{0}$. Now we note that a typical element in $\left(U_{1}^{n+1}\right)^{w^{+}}$has the form

where

$$
\left(\begin{array}{ccc}
1 & \underline{u} & 0 \\
0 & I_{r} & 0 \\
0 & 0 & I_{n-r}
\end{array}\right) \in \mathrm{GL}\left(Y_{r+1}\right) \subset \mathrm{GL}\left(Y_{n+1}\right) \subset G_{n+1}
$$

Moreover,

$$
\underline{u}=\left(u_{2}, u_{3}, \ldots, u_{r}, u_{1}\right) \in F^{r} .
$$

$$
\omega_{n+1, r}\left(w^{+} u g, a\right) \mathcal{F}_{n+1, r}\left(\pi_{K_{H}} \tilde{\phi}\right)\left(\beta_{0}\right)\left(0_{r+1},-\right)=\omega_{n+1, r}(g, a) \mathcal{F}_{n+1, r}\left(\pi_{K_{H}} \tilde{\phi}\right)\left(u^{-1} \circ w^{+} \circ \beta_{0}\right)\left(0_{r+1},-\right)
$$

and it is easy to see that

$$
u^{-1} \circ w^{+} \circ \beta_{0}=\beta_{0}^{\prime}-y_{1} \otimes\left(u_{1} x_{1}^{*}+u_{2} x_{2}^{*}+u_{3} x_{3}^{*}+\cdots+u_{r} x_{r}^{*}\right)
$$


with

$$
\beta_{0}^{\prime}=y_{r+1} \otimes x_{1}^{*}+y_{2} \otimes x_{2}^{*}+\cdots+y_{r} \otimes x_{r}^{*}
$$

Thus, the integral over $\left[\left(U_{1}^{n+1}\right)^{w^{+}}\right]$is simply the Fourier transform in the subspace $Y_{1} \otimes X_{r}^{*}$ (followed by evaluation at 0$)$. In other words, we are looking at the composite

$$
\begin{gathered}
\mathcal{S}\left(Y_{n+1}^{*} \otimes V_{r}\right) \stackrel{\mathcal{F}\left(W_{n+1} \otimes X_{r}^{*}\right) \otimes \mathcal{S}\left(Y_{n+1}^{*} \otimes V_{0}\right)}{\|} \\
\mathcal{S}\left(\left(Y_{1} \oplus Y_{1}^{*}\right) \otimes X_{r}^{*}\right) \otimes \mathcal{S}\left(W_{n} \otimes X_{r}^{*}\right) \otimes \mathcal{S}\left(Y_{n+1}^{*} \otimes V_{0}\right) \\
\downarrow \int_{\left(U_{1}^{n+1}\right)^{w+}}=\text { Fourier transform in } Y_{1} \otimes X_{r}^{*} \\
\mathcal{S}\left(Y_{1}^{*} \otimes\left(X_{r} \oplus X_{r}^{*}\right)\right) \otimes \mathcal{S}\left(W_{n} \otimes X_{r}^{*}\right) \otimes \mathcal{S}\left(Y_{n+1}^{*} \otimes V_{0}\right) \\
\| \\
\mathcal{S}\left(Y_{1}^{*} \otimes V_{r}\right) \otimes \mathcal{S}\left(W_{n} \otimes X_{r}^{*}\right) \otimes \mathcal{S}\left(Y_{n}^{\prime *} \otimes V_{0}\right) \\
\downarrow e v_{0} \text { on } Y_{1}^{*} \otimes V_{r} \\
\mathcal{S}\left(W_{n} \otimes X_{r}^{*}\right) \otimes \mathcal{S}\left(Y_{n}^{\prime *} \otimes V_{0}\right) .
\end{gathered}
$$

But this composite is none other than the map

$$
\text { (evaluation at } \left.0 \text { on } \mathcal{S}\left(Y_{1}^{*} \otimes V_{r}\right)\right) \otimes \mathcal{F}_{n, r} \text {. }
$$

Thus, for $\tilde{\phi}=\phi_{1} \otimes \phi$, we have

$$
\begin{aligned}
& M\left(w^{+}, s\right) f^{n+1, r}\left(s, \pi_{K_{H_{r}}} \tilde{\phi}\right)(g) \\
= & \int_{\mathrm{GL}\left(X_{r}\right)(\mathbb{A})} I_{n-r, 0}\left(\omega(g, a) \mathcal{F}_{n, r}\left(\pi_{K_{H}} \phi\right)\left(\beta_{0}^{\prime}\right)\left(0_{r},-\right)\right)|\operatorname{det}(a)|^{s-\rho_{H_{r}}} d a \\
= & f^{n, r}\left(s, \pi_{K_{H}} \phi\right) .
\end{aligned}
$$

This proves the proposition.

The proposition implies that

$$
E^{n, r}\left(s,\left.M\left(w^{+}, s\right) f^{n+1, r}\left(s, \pi_{K_{H_{r}}} \tilde{\phi}\right)\right|_{G_{n}}\right)=B^{n, r}(s, \phi)
$$

so that we have

$$
\text { (the residue at } \left.s=\rho_{H_{r}} \text { of the second term of }(\underline{9.6})\right)=B_{-1}^{n, r}(\phi) \text {, }
$$

when viewed as an automorphic form on $G_{n}$. This is one of the desired terms in the second term identity. We note that the term $B_{-1}^{n, r}$ is not the leading term of $B^{n, r}(s, \phi)$, which in fact has a pole of order 2 . Thus this pole of order 2 must be cancelled by a pole of order 2 from the term associated to $w^{-}$which we will study next.

The term for $w^{-}$: We now consider the " $w^{-}$-term" of (9.6), namely the Eisenstein series

$$
E^{n, r-1}\left(s-\frac{1}{2},\left.M\left(w^{-}, s\right) f^{n+1, r}\left(s, \pi_{K_{H_{r}}} \tilde{\phi}\right)\right|_{G_{n}}\right) .
$$

We shall apply an argument similar to how we handled the second term on the A-side. Note that we have the standard intertwining operator

$$
M_{n}\left(w_{r-1}, s\right): I_{r-1}^{n}(s, \chi) \longrightarrow I_{r-1}^{n}(-s, \chi),
$$


where $I_{r-1}^{n}$ is as defined in 8.2 and

$$
w_{r-1}=\left(\begin{array}{lll} 
& I_{r-1} \\
& I_{2 n-2 r+2} &
\end{array}\right) .
$$

The functional equation for Eisenstein series says that (9.10) is equal to

$$
E^{n, r-1}\left(\frac{1}{2}-s, M_{n}\left(w_{r-1}, s-\frac{1}{2}\right)\left(\left.M\left(w^{-}, s\right) f^{n+1, r}\left(s, \pi_{K_{H_{r}}} \tilde{\phi}\right)\right|_{G_{n}}\right)\right) .
$$

Then as in [KR3, Lemma 1.2.2], one has

$$
M_{n}\left(w_{r-1}, s-\frac{1}{2}\right)\left(\left.M\left(w^{-}, s\right) f\right|_{G_{n}}\right)=\left.M_{n+1}\left(w_{r}, s\right) f\right|_{G_{n}}
$$

for $f_{s} \in I_{r}^{n+1}(s, \chi)$, where $w_{r}$ is defined analogously to $w_{r-1}$. Thus we are interested in

$$
E^{n, r-1}\left(\frac{1}{2}-s,\left.M_{n+1}\left(w_{r}, s\right) f^{n+1, r}\left(s, \pi_{K_{H_{r}}} \tilde{\phi}\right)\right|_{G_{n}}\right) .
$$

Now we note:

Lemma 9.12.

$$
\begin{aligned}
& E^{n, r-1}\left(\frac{1}{2}-s,\left.M_{n+1}\left(w_{r}, s\right) f^{n+1, r}\left(s, \pi_{K_{H_{r}}} \tilde{\phi}\right)\right|_{G_{n}}\right) \\
= & c_{r}(s) \cdot E^{n, r-1}\left(\frac{1}{2}-s,\left.f^{n+1, r}\left(-s, \pi_{K_{H_{r}}} \tilde{\phi}\right)\right|_{G_{n}}\right) .
\end{aligned}
$$

Proof. We have the functional equation for the Eisenstein series $E^{n+1, r}$ :

$$
\begin{aligned}
& E^{n+1, r}\left(-s, M_{n+1}\left(w_{r}, s\right) f^{n+1, r}\left(s, \pi_{K_{H_{r}}} \tilde{\phi}\right)\right) \\
= & E^{n+1, r}\left(s, f^{n+1, r}\left(s, \pi_{K_{H_{r}}} \tilde{\phi}\right)\right) \\
= & B^{n+1, r}(s, \tilde{\phi}) \\
= & c_{r}(s) \cdot B^{n+1, r}(-s, \tilde{\phi}),
\end{aligned}
$$

where the last equality follows by (3.7). Thus we have:

$$
E^{n+1, r}\left(-s, M_{n+1}\left(w_{r}, s\right) f^{n+1, r}\left(s, \pi_{K_{H_{r}}} \tilde{\phi}\right)\right)=c_{r}(s) \cdot E^{n+1, r}\left(-s, f^{n+1, r}\left(-s, \pi_{K_{H_{r}}} \tilde{\phi}\right)\right) .
$$

Now consider the constant term of both sides along the unipotent radical $U^{n+1}\left(Y_{1}\right)$. By Lemma 8.5, we see that each side is the sum of three automorphic forms on $L^{n+1}\left(Y_{1}\right)=\operatorname{GL}\left(Y_{1}\right) \times G_{n}$. Moreover, for generic $s$, these three automorphic forms can be distinguished from each other by the action of GL $\left(Y_{1}\right)$. Thus we may equate the terms on both sides with the same $\mathrm{GL}\left(Y_{1}\right)$-action. Considering the terms on each side corresponding to the trivial element of the Weyl group, we obtain

$$
E^{n, r-1}\left(\frac{1}{2}-s,\left.M_{n+1}\left(w_{r}, s\right) f^{n+1, r}\left(s, \pi_{K_{H_{r}}} \tilde{\phi}\right)\right|_{G_{n}}\right)=c_{r}(s) \cdot E^{n, r-1}\left(\frac{1}{2}-s,\left.f^{n+1, r}\left(-s, \pi_{K_{H_{r}}} \tilde{\phi}\right)\right|_{G_{n}}\right),
$$

which is the assertion of the lemma.

Remark 9.13. One could directly prove that

$$
M_{n+1}\left(w_{r}, s\right) f^{n+1, r}\left(s, \pi_{K_{H_{r}}} \tilde{\phi}\right)=c_{r}(s) f^{n+1, r}\left(-s, \pi_{K_{H_{r}}} \tilde{\phi}\right)
$$

as sections of the induced representation $I_{r}^{n+1}(-s, \chi)$. Recall from Remark 4.8 at the end of 8 that $f^{n, r}(s, \phi)=\left.F^{n, r}(s, \phi)\right|_{G_{n}}$, where

$$
F^{n, r}(s,-): \omega_{n, r} \longrightarrow I_{r}^{n}(s, \chi) \otimes I_{H_{r}}(-s)
$$


is a $G_{n}(\mathbb{A}) \times H_{r}(\mathbb{A})$-equivariant map defined there. When $n \geq r$, one can establish the identity

$$
M_{n}\left(w_{r}, s\right) F^{n, r}(s,-)=M_{H_{r}}(s) F^{n, r}(-s,-),
$$

and when $\phi$ is $K_{H_{r}}$-invariant, one has:

$$
M_{H_{r}}(s) F^{n, r}(-s, \phi)=c_{r}(s) F^{n, r}(-s, \phi)
$$

on $G_{n}(\mathbb{A}) \times K_{H_{r}}$. Then one can specialize to the situation of $F^{n+1, r}\left(s, \pi_{K_{H_{r}}} \tilde{\phi}\right)$.

By Proposition 4.4, we have:

$$
\left.f^{n+1, r}\left(-s, \pi_{K_{H_{r}}} \tilde{\phi}\right)\right|_{G_{n}}=\alpha_{r} \cdot Z_{1}\left(s-\rho_{H_{r}}, \phi_{1}\right) \cdot f^{n, r-1}\left(-s+\frac{1}{2}, \operatorname{Ik}^{n, r}\left(\pi_{K_{H_{r}}} \phi\right)\right),
$$

where we recall that we are assuming $m=d(n+1)=n+1+\epsilon_{0}$ so that

$$
\rho_{H_{r}}=\frac{n+1-r}{2} \text {. }
$$

From the above lemma, we see that (9.11) is equal to

$$
c_{r}(s) \cdot \alpha_{r} \cdot Z_{1}\left(s-\rho_{H_{r}}, \phi_{1}\right) \cdot B^{n, r-1}\left(\frac{1}{2}-s, \mathrm{Ik}^{n, r}\left(\pi_{K_{H_{r}}} \phi\right)\right),
$$

which is in turn equal to

$$
\frac{c_{r}(s)}{c_{r-1}\left(s-\frac{1}{2}\right)} \cdot \alpha_{r} \cdot Z_{1}\left(s-\rho_{H_{r}}, \phi_{1}\right) \cdot B^{n, r-1}\left(s-\frac{1}{2}, \operatorname{Ik}^{n, r}\left(\pi_{K_{H_{r}}} \phi\right)\right) .
$$

Now we examine the analytic behavior of this function at $s=\rho_{H_{r}}$. Though the answer will turn out to be uniform, it is convenient to consider 3 different cases separately:

(a) Assume first that $r \geq 2$ and $H_{r-1} \neq \mathrm{O}_{1,1}$. Then we have:

- $B^{n, r-1}\left(s-\frac{1}{2}, \mathrm{Ik}^{n, r}\left(\pi_{K_{H_{r}}} \phi\right)\right)$ has a pole of order 1 at $s=\rho_{H_{r}}=\rho_{H_{r-1}}+\frac{1}{2}$, since the case $\left(W_{n}, V_{r-1}\right)$ is in the first term range. Its residue there is precisely $B_{-1}^{n, r-1}\left(\mathrm{Ik}^{n, r}\left(\pi_{K_{H_{r}}} \phi\right)\right)$.

- the Tate zeta integral $Z_{1}\left(s-\rho_{H_{r}}, \phi_{1}\right)$ has a pole of order 1 , and its residue there is equal to $-\phi_{1}(0)=-1$.

- the function $c_{r}(s) / c_{r-1}(s-1 / 2)$ is holomorphic at $s=\rho_{H_{r}}$ and its value at $s=\rho_{H_{r}}$ is equal to $\kappa_{r} / \kappa_{r-1}$ by Lemma 3.4(i).

Thus (9.10) has a pole of order 2 at $s=\rho_{H_{r}}$ and the leading term of (9.10) there is equal to

$$
-\frac{\kappa_{r}}{\kappa_{r-1}} \cdot \alpha_{r} \cdot B_{-1}^{n, r-1}\left(\mathrm{Ik}^{n, r}\left(\pi_{K_{H_{r}}} \phi\right)\right)
$$

while its residue at $s=\rho_{H_{r}}$ (which is the second Laurent coefficient) is

$$
-\frac{\kappa_{r}}{\kappa_{r-1}} \cdot \alpha_{r} \cdot B_{0}^{n, r-1}\left(\mathrm{Ik}^{n, r}\left(\pi_{K_{H_{r}}} \phi\right)\right)+C_{r} \cdot B_{-1}^{n, r-1}\left(\mathrm{Ik}^{n, r}\left(\pi_{K_{H_{r}}} \phi\right)\right)
$$

for some constant $C_{r}$. Moreover, by Theorem 7.3 (the first term identity in the first term range), we see that

To sum up, we have obtained

$$
B_{-1}^{n, r-1}\left(\mathrm{Ik}^{n, r}\left(\pi_{K_{H_{r}}} \phi\right)\right)=\kappa_{r, r-1}^{-1} \cdot A_{-1}^{n, r}(\phi) .
$$

(Residue at $s=\rho_{H_{r}}$ of the third term of (9.6))

$$
=-\frac{\kappa_{r}}{\kappa_{r-1}} \cdot \alpha_{r} \cdot B_{0}^{n, r-1}\left(\operatorname{Ik}^{n, r}\left(\pi_{K_{H_{r}}} \phi\right)\right) \bmod \operatorname{Im} A_{-1}^{n, r}
$$

when viewed as an automorphic form on $G_{n}$. 
(b) When $r=2$ and $H_{r-1}=\mathrm{O}_{1,1}$, we have:

- the Tate zeta integral has a simple pole with residue -1 as above;

- the function $c_{2}(s) / c_{1}(s)$ has a simple pole at $s=\rho_{H_{2}}$ with residue $\kappa_{2} / \kappa_{1}=\kappa_{2}$;

- the term $B^{n, 1}\left(s-\frac{1}{2}, \mathrm{Ik}^{n, 2}\left(\pi_{K_{H_{2}}} \phi\right)\right)$ is holomorphic at $s=\rho_{H_{2}}$.

Thus, (9.10) has a pole of order 2 at $s=\rho_{H_{2}}$ and the leading term of (9.10) there is equal to

$$
-\frac{\kappa_{2}}{\kappa_{1}} \cdot \alpha_{2} \cdot B_{0}^{n, 1}\left(\mathrm{Ik}^{n, 2}\left(\pi_{K_{H_{2}}} \phi\right)\right)
$$

while its residue there is equal to

$$
C \cdot B_{0}^{n, 1}\left(\mathrm{Ik}^{n, 2}\left(\pi_{K_{H_{2}}} \phi\right)\right)-\frac{\kappa_{2}}{\kappa_{1}} \cdot \alpha_{2} \cdot B_{1}^{n, 1}\left(\mathrm{Ik}^{n, 2}\left(\pi_{K_{H_{2}}} \phi\right)\right)
$$

for some constant $C$. Now observe that

$$
B_{1}^{n, 1}\left(\mathrm{Ik}^{n, 2}\left(\pi_{K_{H_{2}}} \phi\right)\right)=0
$$

because $B^{n, 1}\left(s, \mathrm{Ik}^{n, 2}\left(\pi_{K_{H_{2}}} \phi\right)\right)$ is an even function in $s$. Moreover, by Theorem 7.3(i),

$$
B_{0}^{n, 1}\left(\mathrm{Ik}^{n, 2}\left(\pi_{K_{H_{2}}} \phi\right)\right)=\kappa_{2,1}^{-1} \cdot A_{-1}^{n, 2}(\phi) \in \operatorname{Im} A_{-1}^{n, 2} .
$$

Thus, we conclude that when $H_{r-1}=\mathrm{O}_{1,1}$,

$$
\text { (Residue at } \left.s=\rho_{H_{r}} \text { of the third term of (9.6) }\right) \in \operatorname{Im} A_{-1}^{n, r} \text {. }
$$

(c) When $r=1$ so that $H_{r-1}$ is anisotropic, we have

- the Tate zeta integral has a simple pole with residue -1 as above;

- the function $c_{1}(s) / c_{0}(s)$ has a simple pole at $s=\rho_{H_{1}}$ with residue $\kappa_{1}$;

- $B^{n, r-1}\left(s-\frac{1}{2}, \mathrm{Ik}^{n, 1}\left(\pi_{K_{H_{r}}} \phi\right)\right)=I^{n, 0}\left(\mathrm{Ik}^{n, 1}(\phi)\right)$.

Thus, (9.10) has a pole of order 2 at $s=\rho_{H_{1}}$ and the leading term of (9.10) there is equal to

$$
-\frac{\kappa_{1}}{\kappa_{0}} \cdot \alpha_{1} \cdot I^{n, 0}\left(\mathrm{Ik}^{n, 1}(\phi)\right)
$$

while its residue there is equal to

$$
C \cdot I^{n, 0}\left(\mathrm{Ik}^{n, 1}(\phi)\right)
$$

for some constant $C$. On the other hand, Theorem 7.3 (i) implies that

$$
I^{n, 0}\left(\operatorname{Ik}^{n, 1}(\phi)\right)=\kappa_{1,0}^{-1} \cdot A_{-1}^{n, 1}(\phi) \in \operatorname{Im} A_{-1}^{n, 1},
$$

so that

$$
\text { (Residue at } \left.s=\rho_{H_{r}} \text { of the third term of (9.6) }\right) \in \operatorname{Im} A_{-1}^{n, r} \text {. }
$$

9.4. Conclusion of proof. We can now assemble the pieces together and prove Theorem 8.1 in the case $\mathcal{N}=1$. Namely by (9.5), (9.9), (9.14), (9.15) and (9.16), the equation (9.1) is written as

$$
A_{0}^{n, r}(\phi)=B_{-1}^{n, r}(\phi)-\left\{\frac{\kappa_{r}}{\kappa_{r-1}} \cdot \alpha_{r} \cdot B_{0}^{n, r-1}\left(\operatorname{Ik}^{n, r}\left(\pi_{K_{H_{r}}} \phi\right)\right)\right\} \quad \bmod \operatorname{Im} A_{-1}^{n, r}
$$

where $\{\ldots\}$ on the RHS is interpreted to be 0 when $r=1$ or $H_{r-1}=\mathrm{O}_{1,1}$.

Moreover, we observe that the two terms on the $B$-side corresponding to $w^{+}$and $w^{-}$actually have poles of order 2 at $s=\rho_{H_{r}}$. These poles of order 2 must cancel each other, so that we have:

$$
B_{-2}^{n, r}(\phi)=\frac{\kappa_{r}}{\kappa_{r-1}} \cdot \alpha_{r} \cdot B_{*}^{n, r-1}\left(\operatorname{Ik}^{n, r}\left(\pi_{K_{H_{r}}} \phi\right)\right)=\frac{\kappa_{r}}{\kappa_{r-1}} \cdot \alpha_{r} \cdot \kappa_{r, r-1}^{-1} \cdot A_{-1}^{n, r}(\phi),
$$

where $*$ is as in Theorem $7.3(\mathrm{i})$. To complete the proof of Theorem 8.1 , it remains to note: 
Lemma 9.19. The constant

$$
\frac{\kappa_{r}}{\kappa_{r-1}} \cdot \alpha_{r} \cdot \kappa_{r, r-1}^{-1}=1
$$

Proof. If $r=1$, then the identity follows because $\kappa_{0}=1=\alpha_{1}$ and $\kappa_{1,0}=\kappa_{1}$. For $r>1$, the desired identity follows by "Iwasawa decomposition in stages". More precisely, consider the non-maximal parabolic subgroup $R$ of $H_{r}$ stabilizing the flag of isotropic spaces:

$$
X_{1}=\left\langle x_{1}\right\rangle \subset X_{r} \subset V_{r} .
$$

Then $R$ is contained in two maximal parabolic subgroups $P\left(X_{1}\right)$ and $P\left(X_{r}\right)$. Now, relative to the Iwasawa decomposition $H_{r}(\mathbb{A})=R(\mathbb{A}) \cdot K_{H_{r}}$, there is a constant $\gamma$ such that

$$
d h=\gamma \cdot d_{l} r \cdot d k,
$$

where $d_{l} r$ is the left Haar measure of $R(\mathbb{A})$. The constant $\gamma$ can be computed in two ways. First, one may express $d h$ using the Iwasawa decomposition of $H_{r}$ associated to $P\left(X_{1}\right)$ and then using the Iwasawa decomposition of $L\left(X_{1}\right)=\mathrm{GL}\left(X_{1}\right) \times H_{r-1}$ associated to $P^{r-1}\left(X_{r-1}^{\prime}\right)$. This gives

$$
\gamma=\frac{\tau\left(H_{r}\right)}{\tau\left(H_{r-1}\right)} \cdot \kappa_{r, r-1} \cdot \frac{\tau\left(H_{r-1}\right)}{\tau\left(H_{0}\right)} \cdot \kappa_{r-1}=\frac{\tau\left(H_{r}\right)}{\tau\left(H_{0}\right)} \cdot \kappa_{r, r-1} \cdot \kappa_{r-1} .
$$

On the other hand, one may express $d h$ using the Iwasawa decomposition of $H_{r}$ associated to $P\left(X_{r}\right)$ followed by the Iwasawa decomposition associated to the maximal parabolic of $L\left(X_{r}\right)=\operatorname{GL}\left(X_{r}\right)$ stabilizing the line $X_{1}$. This yields

$$
\gamma=\frac{\tau\left(H_{r}\right)}{\tau\left(H_{0}\right)} \cdot \kappa_{r} \cdot \alpha_{r}
$$

which proves the lemma.

If one uses the result of the lemma, one can see that (9.17) and (9.18) are written as in Theorem 8.1 This completes the base step in the inductive proof of Theorem 8.1 .

\section{Proof of Theorem 8.1; Inductive Step}

In this section, we complete the proof of Theorem 8.1 by establishing the inductive step of the argument. Thus, suppose we want to prove Theorem 8.1 for the pair $\left(W_{n}, V_{r}\right)$ with associated $\mathcal{N}_{0}=m-d(n)$. We consider the pair $\left(W_{n+1}, V_{r}\right)$ with associated $\mathcal{N}=m-d(n+1)=\mathcal{N}_{0}-1 \geq 1$, so that we may appeal to the induction hypothesis.

As in the proof of the base case, we fix $\phi_{1} \in \mathcal{S}\left(y_{1}^{*} \otimes V_{r}\right)(\mathbb{A})$ satisfying the conditions in 9.1 . Then for arbitrary $\phi \in \mathcal{S}\left(Y_{n}^{\prime *} \otimes V_{r}\right)(\mathbb{A})$, set $\tilde{\phi}=\phi_{1} \otimes \phi$. Applying the induction hypothesis, we have:

$$
A_{-1}^{n+1, r}(\tilde{\phi})=B_{-2}^{n+1, r}(\tilde{\phi}) \text {, }
$$

and

$$
A_{0}^{n+1, r}(\tilde{\phi})=B_{-1}^{n+1, r}(\tilde{\phi})-\kappa_{r, r^{\prime}} \cdot B_{0}^{n+1, r^{\prime}}\left(\operatorname{Ik}^{n+1, r}\left(\pi_{K_{H_{r}}} \tilde{\phi}\right)\right)+A_{-1}^{n+1, r}(\varphi)
$$

for some $\varphi \in \mathcal{S}\left(Y_{n+1}^{*} \otimes V_{r}\right)(\mathbb{A})$, where $r^{\prime}$ satisfies $m_{0}+r+r^{\prime}=d(n+1)$. Note that the second term on the RHS of (10.2) is always present here, because $H_{r^{\prime}}$ cannot be anisotropic or equal to $\mathrm{O}_{1,1}$ in the present setting. Indeed, the complementary space of $V_{r}$ with respect to $W_{n}$ is $V_{r^{\prime}-1}$ so that $r^{\prime} \geq 1$ and $H_{r^{\prime}}$ is not anisotropic. On the other hand, if $H_{r}=\mathrm{O}_{r, r}$ (so that $m_{0}=0$ and $\epsilon_{0}=1$ ), then $r+r^{\prime}=n+2$, and with our assumption that $r \leq n$, we see that $r^{\prime}>1$ in the case $\epsilon_{0}=1$ and $m_{0}=0$. 
10.1. First term identity. Let us examine (10.1) first. Computing the constant term along $U\left(Y_{1}\right)$, we have:

$$
A_{-1}^{n+1, r}(\tilde{\phi})_{U\left(Y_{1}\right)}=B_{-2}^{n+1, r}(\tilde{\phi})_{U\left(Y_{1}\right)} .
$$

Using Lemma 8.5 to compute the constant terms on both sides, we shall extract the terms on both sides where $\mathrm{GL}\left(Y_{1}\right) \subset L\left(Y_{1}\right)$ acts by the character $\chi \cdot|-|^{m / 2}$. Then on the B-side, we see that only the term for $w^{+}$contributes, and Proposition 9.8 gives:

$$
\text { (the } \left.\chi \cdot|-|^{m / 2} \text {-part of } B_{-2}^{n+1, r}(\tilde{\phi})_{U\left(Y_{1}\right)}\right)=B_{-2}^{n, r}(\phi) \text {. }
$$

On the other hand, on the A-side, only the first term in Lemma 8.5(ii) contributes, giving

$$
\text { (the } \left.\chi \cdot|-|^{m / 2} \text {-part of } A_{-1}^{n+1, r}(\phi)_{U\left(Y_{1}\right)}\right)=A_{-1}^{n, r}(\phi) \text {. }
$$

This proves Theorem $8.1(\mathrm{i})$.

10.2. Second term identity. Now consider (10.2). Computing the constant term along $U\left(Y_{1}\right)$ gives:

$$
\begin{aligned}
A_{0}^{n+1, r}(\tilde{\phi})_{U\left(Y_{1}\right)} & =B_{-1}^{n+1, r}(\tilde{\phi})_{U\left(Y_{1}\right)} \\
& -\kappa_{r, r^{\prime}} \cdot B_{0}^{n+1, r^{\prime}}\left(\operatorname{Ik}^{n+1, r}\left(\pi_{K_{H_{r}}} \tilde{\phi}\right)\right)_{U\left(Y_{1}\right)} \\
& +A_{-1}^{n+1, r}(\varphi)_{U\left(Y_{1}\right)} .
\end{aligned}
$$

Each constant term in this identity can be computed by using Lemma 8.5 just as we did for the base step, and is written as a sum of two or three automorphic forms on $\operatorname{GL}\left(Y_{1}\right) \times G_{n}$. Then by extracting the terms with $\operatorname{GL}\left(Y_{1}\right)$ acting via $\chi \cdot|-|^{m / 2}$, we can deduce the desired second term identity. To be specific, for the LHS of (10.3), only the trivial Weyl group element contributes, and we have

$$
\left(\text { the } \chi \cdot|-|^{m / 2} \text {-part of } A_{0}^{n+1, r}(\tilde{\phi})_{U\left(Y_{1}\right)}\right)=A_{0}^{n, r}(\phi) \text {, }
$$

when viewed as an automorphic form on $G_{n}$. For the first term of the RHS of (10.3), only the Weyl group element $w^{+}$contributes and we have

$$
\left(\text { the } \chi \cdot|-|^{m / 2} \text {-part of } B_{-1}^{n+1, r}(\tilde{\phi})_{U\left(Y_{1}\right)}\right)=B_{-1}^{n, r}(\phi) \text {. }
$$

For the third term of the RHS of (10.3), we have

$$
\left(\text { the } \chi \cdot|-|^{m / 2} \text {-part of } A_{-1}^{n+1, r}(\varphi)_{U\left(Y_{1}\right)}\right) \in \operatorname{Im} A_{-1}^{n, r}
$$

as we have noted above for the first term identity.

Finally for the second term of the RHS of (10.3), only the Weyl group element $w^{-}$contributes and one sees that

$$
\begin{aligned}
& \left(\text { the } \chi \cdot|-|^{m / 2} \text {-part of } B_{0}^{n+1, r^{\prime}}\left(\mathrm{Ik}^{n+1, r}\left(\pi_{K_{H_{r}}} \tilde{\phi}\right)\right)_{U\left(Y_{1}\right)}\right) \\
& \left.=\operatorname{Val}_{s=\rho_{H_{r^{\prime}}}} E^{n, r^{\prime}-1}\left(s-\frac{1}{2},\left.M\left(w^{-}, s\right) f^{n+1 . r^{\prime}}\left(s, \mathrm{Ik}^{n+1, r}\left(\pi_{K_{H_{r}}} \tilde{\phi}\right)\right)\right|_{G_{n}}\right)\right) .
\end{aligned}
$$

This is the most complex case. First by the arguments in the $w^{-}$-term for the base step and Lemma 9.12, we can write the Eisenstein series in (10.7) as

$$
\begin{aligned}
& E^{n, r^{\prime}-1}\left(s-\frac{1}{2},\left.M\left(w^{-}, s\right) f^{n+1 . r^{\prime}}\left(s, \operatorname{Ik}^{n+1, r}\left(\pi_{K_{H_{r}}} \tilde{\phi}\right)\right)\right|_{G_{n}}\right) \\
= & c_{r^{\prime}}(s) \cdot E^{n, r^{\prime}-1}\left(\frac{1}{2}-s, f^{n+1 \cdot r^{\prime}}\left(-s,\left.\operatorname{Ik}^{n+1, r}\left(\pi_{K_{H_{r}}} \tilde{\phi}\right)\right|_{G_{n}}\right)\right) .
\end{aligned}
$$


Noting that

$$
\mathrm{Ik}^{n+1, r}\left(\pi_{K_{H_{r}}} \tilde{\phi}\right)=\mathrm{Ik}^{1, r, r^{\prime}}\left(\phi_{1}\right) \otimes \mathrm{Ik}^{n, r, r^{\prime}}\left(\pi_{K_{H_{r}}} \phi\right)
$$

is $K_{H_{r^{\prime}}}$-invariant, Proposition 4.4 implies that (10.8) is equal to

$$
\begin{aligned}
c_{r^{\prime}}(s) \cdot \alpha_{r^{\prime}} \cdot Z_{1}\left(s-\left(n+1-r^{\prime}\right)+\rho_{H_{r^{\prime}}}, \mathrm{Ik}^{1, r, r^{\prime}}\left(\phi_{1}\right)\right) \\
\cdot E^{n, r^{\prime}-1}\left(\frac{1}{2}-s, f^{n, r^{\prime}-1}\left(\frac{1}{2}-s, \mathrm{Ik}^{n, r^{\prime}, r^{\prime}-1}\left(\mathrm{Ik}^{n, r, r^{\prime}}\left(\pi_{K_{H_{r}}} \phi\right)\right)\right)\right) \\
=c_{r^{\prime}}(s) \cdot \alpha_{r^{\prime}} \cdot Z_{1}\left(s-\left(n+1-r^{\prime}\right)+\rho_{H_{r^{\prime}}}, \mathrm{Ik}^{1, r, r^{\prime}}\left(\phi_{1}\right)\right) \\
\cdot E^{n, r^{\prime}-1}\left(\frac{1}{2}-s, f^{n, r^{\prime}-1}\left(\frac{1}{2}-s, \mathrm{Ik}^{n, r, r^{\prime}-1}\left(\pi_{K_{H_{r}}} \phi\right)\right)\right) .
\end{aligned}
$$

Note that since $m_{0}+r+r^{\prime}=d(n+1)$, we have $m_{0}+r+\left(r^{\prime}-1\right)=d(n)$, so that $V_{r}$ and $V_{r^{\prime}-1}$ are complementary with respect to $W_{n}$ and

$$
\mathrm{Ik}^{n, r, r^{\prime}-1}=\mathrm{Ik}^{n, r}
$$

Thus (10.9) is equal to

$$
c_{r^{\prime}}(s) \cdot \alpha_{r^{\prime}} \cdot Z_{1}\left(s-\left(n+1-r^{\prime}\right)+\rho_{H_{r^{\prime}}}, \mathrm{Ik}^{1, r, r^{\prime}}\left(\phi_{1}\right)\right) \cdot B^{n, r^{\prime}-1}\left(\frac{1}{2}-s, \mathrm{Ik}^{n, r}\left(\pi_{K_{H_{r}}} \phi\right)\right)
$$

which is in turn equal to

$$
\frac{c_{r^{\prime}}(s)}{c_{r^{\prime}-1}\left(s-\frac{1}{2}\right)} \cdot \alpha_{r^{\prime}} \cdot Z_{1}\left(s-\left(n+1-r^{\prime}\right)+\rho_{H_{r^{\prime}}} \operatorname{Ik}^{1, r, r^{\prime}}\left(\phi_{1}\right)\right) \cdot B^{n, r^{\prime}-1}\left(s-\frac{1}{2}, \operatorname{Ik}^{n, r}\left(\pi_{K_{H_{r}}} \phi\right)\right) .
$$

We thus need to consider the zeroth term in the Laurent expansion of (10.10) at $s=\rho_{H_{r^{\prime}}}=\rho_{H_{r^{\prime}-1}}+1 / 2$.

As in the argument for the base step in the previous section, it will be convenient to consider different cases separately. If $H_{r^{\prime}-1}$ is anisotropic or equal to $\mathrm{O}_{1,1}$, then as we have seen in the previous section, $B^{n, r^{\prime}-1}\left(s-\frac{1}{2}, \mathrm{Ik}^{n, r}\left(\pi_{K_{H_{r}}} \phi\right)\right)$ is holomorphic at $s=\rho_{H_{r^{\prime}}}$ with vanishing first derivative there, whereas the product of the other terms has a simple pole (arising from the pole of $c_{r^{\prime}}(s) / c_{r^{\prime}-1}\left(s-\frac{1}{2}\right)$ at $s=\rho_{H_{r^{\prime}}}$ ). Thus, on taking the zeroth Laurent coefficient, one obtains

$$
C \cdot B_{0}^{n, r^{\prime}-1}\left(\mathrm{Ik}^{n, r}\left(\pi_{K_{H_{r}}} \phi\right)\right) \in \operatorname{Im} A_{-1}^{n, r}
$$

by Theorem $7.3(\mathrm{i})$. This completes the proof of the inductive step for these cases.

Finally, we assume that $H_{r^{\prime}-1} \neq \mathrm{O}_{1,1}$ is isotropic. Then we know from Lemma 3.4 (i) that $c_{r^{\prime}}(s) / c_{r^{\prime}-1}(s-$ $1 / 2)$ is holomorphic with value $\kappa_{r^{\prime}} / \kappa_{r^{\prime}-1}$. The zeta integral is evaluated at $-\left(r-r^{\prime}\right) \leq-1$ where it is holomorphic, whereas $B^{n, r^{\prime}-1}\left(s-\frac{1}{2}, \mathrm{Ik}^{n, r}\left(\pi_{K_{H_{r}}} \phi\right)\right)$ has a pole of order 1 . Taking the zeroth term in the Laurent expansion, (10.10) becomes

$$
\begin{aligned}
\kappa_{r^{\prime}} / \kappa_{r^{\prime}-1} \cdot \alpha_{r^{\prime}} \cdot Z_{1}\left(-\left(r-r^{\prime}\right), \mathrm{Ik}^{1, r, r^{\prime}}\left(\phi_{1}\right)\right) \cdot & B_{0}^{n, r^{\prime}-1}\left(\mathrm{Ik}^{n, r}\left(\pi_{K_{H_{r}}} \phi\right)\right) \\
& \bmod \operatorname{Im}\left(B_{-1}^{n, r^{\prime}-1} \circ \mathrm{Ik}^{n, r} \circ \pi_{K_{H_{r}}}\right) .
\end{aligned}
$$

By the first term identity in Theorem 7.3 and Lemma 9.19, (10.11) becomes

$$
\kappa_{r^{\prime}, r^{\prime}-1} \cdot Z_{1}\left(-\left(r-r^{\prime}\right), \mathrm{Ik}^{1, r, r^{\prime}}\left(\phi_{1}\right)\right) \cdot B_{0}^{n, r^{\prime}-1}\left(\operatorname{Ik}^{n, r}\left(\pi_{K_{H_{r}}} \phi\right)\right) \quad \bmod \operatorname{Im} A_{-1}^{n, r} .
$$

It remains to obtain a better handle of the term

$$
Z_{1}\left(-\left(r-r^{\prime}\right), \mathrm{Ik}^{1, r, r^{\prime}}\left(\phi_{1}\right)\right):=\mathrm{Val}_{s=0} \int_{\mathbb{A}_{E}^{\times}} \int_{\mathbb{A}_{E}^{r-r^{\prime}}} \phi_{1}\left(\begin{array}{c}
x_{r-r^{\prime}} \\
t \\
0_{m-r+r^{\prime}-1}
\end{array}\right)|t|^{s-\left(r-r^{\prime}\right)} d x_{r-r^{\prime}} d^{\times} t .
$$

This is given by the following lemma (see [11, Pg. 231, (7.1)]). 
Lemma 10.13. Let $\varphi \in \mathcal{S}\left(\mathbb{A}^{k}\right)$ with $k \geq 2$ be such that $\varphi$ is $K$-invariant, where $K \subset \mathrm{GL}_{k}(\mathbb{A})$ is a maximal compact subgroup and where $\mathrm{GL}_{k}(\mathbb{A})$ acts on $\mathcal{S}\left(\mathbb{A}^{k}\right)$ by $g \cdot \varphi(x)=\varphi\left(g^{-1} \cdot x\right)$. Then

$$
\operatorname{Val}_{s=0}\left(\int_{t \in \mathbb{A}^{\times}} \int_{x_{k-1} \in \mathbb{A}^{k-1}} \varphi\left(\begin{array}{c}
x_{k-1} \\
t
\end{array}\right) \cdot|t|^{s-(k-1)} d x_{k-1} d^{\times} t\right)=\frac{\varphi(0)}{\alpha_{k}}
$$

where the constant $\alpha_{k}$ is as defined in (4.5), and where the double integral on the LHS is absolutely convergent when $\operatorname{Re}(s) \gg 0$ and has a meromorphic continuation to $\mathbb{C}$.

Proof. Let $z(s, \varphi)$ denote the double integral on the LHS. Choose a function $\varphi^{\prime} \in \mathcal{S}\left(M_{k \times(k-1)}(\mathbb{A})\right)$ that is left invariant by $K$ with $\varphi^{\prime}(0)=1$. Construct $\Phi=\varphi^{\prime} \otimes \varphi \in \mathcal{S}\left(M_{k \times k}(\mathbb{A})\right)$ and consider the zeta integral $Z_{k}(s, \Phi)=\int_{G L_{k}(A)} \Phi(A)|\operatorname{det} A|^{s} d A$. There is a measure decomposition $d A=\alpha_{k}|t|^{-(k-1)} d x_{k-1} d B d^{\times} t d \lambda$ with respect to the Iwasawa decomposition $A=\left({ }_{{ }^{t} x_{k-1} t}^{B}\right) \lambda$, where $x_{k-1} \in \mathbb{A}^{k-1}, B \in \mathrm{GL}_{k-1}(\mathbb{A}), t \in \mathbb{A}^{\times}$, and $\lambda \in K$. Let $\varphi_{1}^{\prime}$ be the restriction of $\varphi^{\prime}$ to $M_{(k-1) \times(k-1)}(\mathbb{A}) \times\left\{0_{k-1}\right\}$, then

$$
\begin{aligned}
Z_{k}(\Phi, s) & =\int_{\mathrm{GL}_{k}(\mathbb{A})} \Phi\left({ }^{t} A\right)|\operatorname{det} A|^{s} d A \\
& =\alpha_{k} \int_{K} \int_{\mathrm{GL}_{k-1}(\mathbb{A})} \int_{\mathbb{A}^{\times}} \int_{\mathbb{A}^{k-1}} \Phi\left({ }^{t} \lambda\left(\begin{array}{cc}
{ }^{t} B & x_{k-1} \\
t
\end{array}\right)\right)|\operatorname{det} B|^{s}|t|^{s-(k-1)} d x_{k-1} d B d^{\times} t d \lambda \\
& =\alpha_{k} \int_{\mathrm{GL}_{k-1}(\mathbb{A})} \int_{\mathbb{A}^{\times}} \int_{\mathbb{A}^{k-1}} \Phi\left(\begin{array}{c}
B x_{k-1} \\
t
\end{array}\right)|\operatorname{det} B|^{s}|t|^{s-(k-1)} d x_{k-1} d B d^{\times} t \\
& =\alpha_{k} Z_{k-1}\left(\varphi_{1}^{\prime}, s\right) z(s, \varphi) .
\end{aligned}
$$

Because $Z_{k}(\Phi, s)$ and $Z_{k-1}\left(\varphi_{1}^{\prime}, s\right)$ both have a simple pole at $s=0$ and the residues are $-\Phi(0),-\varphi_{1}^{\prime}(0)$ respectively (c.f. section 4.1), we have

$$
\operatorname{Val}_{s=0} z(s, \varphi)=\frac{-\Phi(0)}{-\varphi_{1}^{\prime}(0) \alpha_{k}}=\frac{\varphi(0)}{\alpha_{k}} .
$$

Applying the lemma, we deduce that

$$
Z_{1}\left(-\left(r-r^{\prime}\right), \operatorname{Ik}^{1, r, r^{\prime}}\left(\phi_{1}\right)\right)=\frac{1}{\alpha_{r-r^{\prime}+1}},
$$

so that (10.12) is equal to

$$
\frac{\kappa_{r^{\prime}, r^{\prime}-1}}{\alpha_{r-r^{\prime}+1}} \cdot B_{0}^{n, r^{\prime}-1}\left(\operatorname{Ik}^{n, r}\left(\pi_{K_{H_{r}}} \phi\right)\right) \bmod \operatorname{Im} A_{-1}^{n, r} .
$$

It remains to show that

$$
\frac{\kappa_{r^{\prime}, r^{\prime}-1}}{\alpha_{r-r^{\prime}+1}}=\frac{\kappa_{r, r^{\prime}-1}}{\kappa_{r, r^{\prime}}} .
$$

This again follows by "Iwasawa decomposition in stages". Namely, we consider the parabolic subgroup $R$ of $H_{r}$ stabilizing the flag

$$
X_{r-r^{\prime}} \subset X_{r-r^{\prime}+1} \subset V_{r} .
$$

Then $R$ is contained in the maximal parabolic subgroups $P\left(X_{r-r^{\prime}}\right)$ and $P\left(X_{r-r^{\prime}+1}\right)$. By the same argument as in Lemma 9.19, one obtains:

$$
\kappa_{r, r^{\prime}} \cdot \kappa_{r^{\prime}, r^{\prime}-1}=\kappa_{r, r^{\prime}-1} \cdot \alpha_{r-r^{\prime}+1},
$$

which is the desired identity. 
Hence (10.12) (which is actually (10.7) ) becomes

$$
\frac{\kappa_{r, r^{\prime}-1}}{\kappa_{r, r^{\prime}}} \cdot B_{0}^{n, r^{\prime}-1}\left(\mathrm{Ik}^{n, r}\left(\pi_{K_{H_{r}}} \phi\right)\right) \quad \bmod \operatorname{Im} A_{-1}^{n, r} .
$$

Hence if one combines the four equations (10.4) (10.5) (10.6) and (10.14) with (10.3), one can see that one has the desired second term identity for the pair $\left(W_{n}, V_{r}\right)$ with associated $\mathcal{N}_{0}=m-d(n)$, which completes the inductive step.

Hence the proof of Theorem 8.1 is now complete.

\section{Rallis Inner product Formula}

In this section, we use the first and second term identities of the regularized Siegel-Weil formula to derive the Rallis inner product formula and prove our non-vanishing theorem of global theta lifts.

11.1. Dual Pairs. As before, let $V_{r}$ be an $m$-dimensional $\epsilon$-Hermitian space over $E$ with Witt index $r$ and anisotropic kernel $V_{0}$ of dimension $m_{0}$, so that $m=m_{0}+2 r$. Let $U_{n}$ be an $n$-dimensional $-\epsilon$-Hermitian space over $E$. Then the pair of spaces $\left(U_{n}, V_{r}\right)$ determines the dual reductive pair $G\left(U_{n}\right) \times H\left(V_{r}\right)$ as follows.

\begin{tabular}{|c|c|c|c|}
\hline$\epsilon_{0}$ & & $G\left(U_{n}\right)$ & $H\left(V_{r}\right)$ \\
\hline 0 & & $\mathrm{U}_{n}$ & $\mathrm{U}_{m}$ \\
\hline \multirow{2}{*}{-1} & $n$ even & $\mathrm{O}_{n}$ & $\mathrm{Sp}_{m}$ \\
\cline { 2 - 4 } & $n$ odd & $\mathrm{O}_{n}$ & $\mathrm{Mp}_{m}$ \\
\hline \multirow{2}{*}{1} & $m$ even & $\mathrm{Sp}_{n}$ & $\mathrm{O}_{m}$ \\
\cline { 2 - 4 } & $m$ odd & $\mathrm{Mp}_{n}$ & $\mathrm{O}_{m}$ \\
\hline
\end{tabular}

Observe that, while we have always taken $H\left(V_{r}\right)$ to be a linear group in the earlier sections of the paper, we have now allowed $H\left(V_{r}\right)$ to be the metaplectic group.

11.2. Theta lifts. To define the global theta lifting from $G\left(U_{n}\right)$ to $H\left(V_{r}\right)$, one needs to choose some auxiliary data for the splitting of metaplectic covers so as to define the Weil representation of $G\left(U_{n}\right) \times$ $H\left(V_{r}\right)$. Namely, one fixes a pair of Hecke characters $\left(\chi_{U}, \chi_{V}\right)$ as follows:

$$
\begin{aligned}
& \chi_{U}= \begin{cases}\text { a Hecke character of } \mathbb{A}_{E^{\times}} \text {such that }\left.\chi_{W}\right|_{\mathbb{A}^{\times}}=\chi_{E}^{n} & \text { if } \epsilon_{0}=0, \\
\text { the quadratic Hecke character of } \mathbb{A}^{\times} \text {associated to } \operatorname{disc} U_{n} & \text { if } \epsilon_{0}=-1, \\
\text { the trivial character of } \mathbb{A}^{\times} & \text {if } \epsilon_{0}=1 ;\end{cases} \\
& \chi_{V}= \begin{cases}\text { a Hecke character of } \mathbb{A}_{E^{\times}} \text {such that }\left.\chi_{V}\right|_{\mathbb{A}^{\times}}=\chi_{E}^{m} & \text { if } \epsilon_{0}=0, \\
\text { the trivial character of } \mathbb{A}^{\times} & \text {if } \epsilon_{0}=-1, \\
\text { the quadratic Hecke character of } \mathbb{A}^{\times} \text {associated to } \operatorname{disc} V_{r} & \text { if } \epsilon_{0}=1 .\end{cases}
\end{aligned}
$$

Having fixed $\left(\chi_{U}, \chi_{V}\right)$, one then has the associated Weil representation $\omega_{\psi, \chi_{U}, \chi_{V}}$ of $G\left(U_{n}\right) \times H\left(V_{r}\right)$. If $U_{n} \otimes_{E} V_{r}=\mathbb{X} \oplus \mathbb{Y}$ is a Witt decomposition of the symplectic space $U_{n} \otimes_{E} V_{r}$, then $\omega_{\psi, \chi_{U}, \chi_{V}}$ may be realized on the Schwartz space $\mathcal{S}(\mathbb{X})(\mathbb{A})$. One then has the usual automorphic realization

$$
\theta: \omega_{\psi, \chi_{U}, \chi_{V}} \longrightarrow\left\{\text { Functions on }\left[G\left(U_{n}\right)\right] \times\left[H\left(V_{r}\right)\right]\right\}
$$

given by

$$
\theta(\phi)(g, h)=\sum_{x \in \mathbb{X}(F)} \omega_{\psi, \chi_{U}, \chi_{V}}(g, h) \phi(x)
$$


for $(g, h) \in G\left(U_{n}\right)(\mathbb{A}) \times H\left(V_{r}\right)(\mathbb{A})$ and $\phi \in \mathcal{S}(\mathbb{X})(\mathbb{A})$. For a cuspidal representation $\pi$ of $G\left(U_{n}\right)$, we consider its global theta lift $\Theta_{n, r}(\pi)$ to $H\left(V_{r}\right)$, so that $\Theta_{n, r}(\pi)$ is the automorphic subrepresentation of $H\left(V_{r}\right)$ spanned by the automorphic forms

$$
\theta_{n, r}(\phi, f)(h)=\int_{\left[G\left(U_{n}\right)\right]} \theta(\phi)(g, h) \cdot \overline{f(g)} d g
$$

for $\phi \in \omega_{\psi, \chi_{U}, \chi_{V}}$ and $f \in \pi$.

We assume that $\Theta_{n, j}(\pi)=0$ for $j<r$. Then a well-known result of Rallis [R1] says that $\Theta_{n, r}(\pi)$ is contained in the space of cusp forms on $H\left(V_{r}\right)$. We are interested in detecting whether $\Theta_{n, r}(\pi)$ is nonzero.

11.3. Doubling See-Saw. To detect if $\Theta_{n, r}(\pi)$ is nonzero, we compute the inner product

$$
\left\langle\theta_{n, r}\left(\phi_{1}, f_{1}\right), \theta_{n, r}\left(\phi_{2}, f_{2}\right)\right\rangle
$$

for $\phi_{i} \in \omega_{\psi, \chi_{U}, \chi_{V}}$ and $f_{i} \in \pi$. The mechanism for this computation is the following doubling see-saw diagram

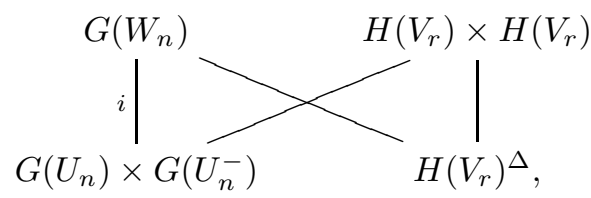

where $U_{n}^{-}$is the space $U_{n}$ with the form scaled by -1 , and

$$
W_{n}=U_{n} \oplus\left(U_{n}^{-}\right)
$$

is the split $\epsilon$-Hermitian space of dimension $2 n$. Indeed, we choose a Witt decomposition of $W_{n}$ to be

$$
W_{n}=Y_{n} \oplus Y_{n}^{*}
$$

with

$$
Y_{n}=U_{n}^{\Delta}=\left\{(u, u): u \in U_{n}\right\}
$$

and

$$
Y_{n}^{*}=U_{n}^{\nabla}=\left\{(u,-u): u \in U_{n}\right\} .
$$

The Weil representation $\omega_{\psi, \chi_{V}, W_{n}, V_{r}}$ of $G\left(W_{n}\right) \times H\left(H_{r}\right)^{\Delta}$ (which is what we have denoted by $\omega_{n, r}$ in the previous sections) can be realized on $\mathcal{S}\left(Y_{n}^{*} \otimes V_{r}\right)$ such that $H\left(V_{r}\right)^{\Delta}$ acts by

$$
\omega_{\psi, \chi_{V}, W_{n}, V_{r}}(h) \phi(x)=\varphi\left(h^{-1} \cdot x\right) .
$$

Thus, the branch of the see-saw involving the dual pair

$$
G\left(W_{n}\right) \times H\left(V_{r}\right)^{\Delta}
$$

gives rise to the (regularized) theta integral which we studied in the earlier parts of this paper. By [K2] and HKS, one knows that

$$
\left.\omega_{\psi, \chi_{V}, W_{n}, V_{r}}\right|_{G\left(U_{n}\right) \times G\left(U_{n}\right)}=\left.\omega_{\psi, \chi_{V}, U_{n}, V_{r}} \otimes\left(\omega_{\psi, \chi_{V}, U_{n}, V_{r}}^{\vee} \cdot\left(\chi_{V} \circ \operatorname{det}\right)\right)\right|_{G\left(U_{n}\right) \times G\left(U_{n}\right)} .
$$

Indeed, there is an isomorphism [Li, Pg. 182]

$$
\delta: \omega_{\psi, \chi_{V}, U_{n}, V_{r}} \otimes\left(\omega_{\psi, \chi_{V}, U_{n}, V_{r}}^{\vee} \cdot\left(\chi_{V} \circ \operatorname{det}\right)\right) \longrightarrow \omega_{\psi, \chi_{V}, W_{n}, V_{r}}
$$

such that

for $\phi_{i} \in \mathcal{S}(\mathbb{X})(\mathbb{A})$.

$$
\delta\left(\phi_{1} \otimes \overline{\phi_{2}}\right)(0)=\left\langle\phi_{1}, \phi_{2}\right\rangle
$$


11.4. Inner product. We remind the reader of the different possible ranges for the triple $(n, m, r)$ :

\begin{tabular}{|c|c|c|}
\hline \multirow{2}{*}{$m \leq d(n)$} & $r=0$ & convergent range \\
\cline { 2 - 3 } & $r>0$ & boundary/first term range \\
\hline \multirow{2}{*}{$d(n)<m \leq 2 \cdot d(n)$} & $m \leq d(n)+r$ & second term range \\
\cline { 2 - 3 } & $m>d(n)+r$ & convergent range \\
\hline$m>2 \cdot d(n)$ & & convergent range \\
\hline
\end{tabular}

Since we are primarily interested in the second term range, we shall assume henceforth that

$$
d(n)<m \leq 2 \cdot d(n) .
$$

This implies that

$$
0<s_{m, n}=\frac{m-d(n)}{2} \leq \frac{d(n)}{2} .
$$

Now the see-saw identity for the doubling see-saw gives:

Proposition 11.1. Suppose that $d(n)<m \leq 2 \cdot d(n)$. Let $\pi$ be an irreducible cuspidal automorphic representation of $G\left(U_{n}\right)$ such that $\Theta_{n, j}(\pi)=0$ for $j<r$, so that $\Theta_{n, r}(\pi)$ is cuspidal.

(i) If the triple $(n, m, r)$ is in the second term range and $r \leq n$, then one has

$$
\begin{aligned}
& \left\langle\theta_{n, r}\left(\phi_{1}, f_{1}\right), \theta_{n, r}\left(\phi_{2}, f_{2}\right)\right\rangle \\
& =\tau\left(H_{r}\right) \cdot \int_{\left[G\left(U_{n}\right) \times G\left(U_{n}\right)\right]} B_{-1}^{n, r}\left(\delta\left(\phi_{1} \otimes \bar{\phi}_{2}\right)\right)\left(g_{1}, g_{2}\right) \cdot \overline{f_{1}\left(g_{1}\right)} \cdot f_{2}\left(g_{2}\right) \cdot \chi_{V}^{-1}\left(\operatorname{det}\left(g_{2}\right)\right) d g_{1} d g_{2} \\
& =[E: F] \cdot \int_{\left[G\left(U_{n}\right) \times G\left(U_{n}\right)\right]} A_{0}^{n, r}\left(\delta\left(\phi_{1} \otimes \bar{\phi}_{2}\right)\right)\left(g_{1}, g_{2}\right) \cdot \overline{f_{1}\left(g_{1}\right)} \cdot f_{2}\left(g_{2}\right) \cdot \chi_{V}^{-1}\left(\operatorname{det}\left(g_{2}\right)\right) d g_{1} d g_{2}
\end{aligned}
$$

for $\phi_{i} \in \omega_{\psi, \chi_{U}, \chi_{V}}$ and $f_{i} \in \pi$.

(ii) If the triple $(n, m, r)$ is in the convergent range, then one has the same identity relating the first term and the third term in (i).

Proof. (i) The first equality is proved in the same way as [GT, Prop. 6.1]. The second equality is proved in the same way as [GT, Prop. 6.3] by using the second term identity of Theorem 8.1.

(ii) This similarly follows from the Siegel-Weil formula ([KR1, KR2] and [13]) in the convergent case.

11.5. Doubling zeta integral. The last integral in (i) of the above proposition is the so-called doubling zeta integral. More precisely, for $f_{i} \in \pi$ and $\Phi(s)$ a holomorphic section of $I_{n}^{n}\left(s, \chi_{V}\right):=\operatorname{Ind}_{P\left(Y_{n}\right)}^{G\left(W_{n}\right)}\left(\chi_{V} \circ\right.$ $\operatorname{det})|\operatorname{det}|^{s}$, one sets

$$
Z\left(s, \Phi, f_{1}, f_{2}\right)=\int_{\left[G\left(U_{n}\right)\right] \times\left[G\left(U_{n}\right)\right]} E(s, \Phi)\left(g_{1}, g_{2}\right) \cdot \overline{f_{1}\left(g_{1}\right)} \cdot f_{2}\left(g_{2}\right) \cdot \chi_{V}^{-1}\left(\operatorname{det}\left(g_{2}\right)\right), d g_{1} d g_{2} .
$$

By [PS-R], this converges for $\operatorname{Re}(s) \gg 0$ and extends to a meromorphic function on $\mathbb{C}$. Thus Proposition 11.1 can be stated as

Proposition 11.2. Assume that $\pi$ is as in Proposition 11.1. Then for $\phi_{i} \in \omega_{\psi, \chi_{U}, \chi_{V}}$ and $f_{i} \in \pi$, one has

$$
\left\langle\theta_{n, r}\left(\phi_{1}, f_{1}\right), \theta_{n, r}\left(\phi_{2}, f_{2}\right)\right\rangle=[E: F] \cdot \operatorname{Val}_{s=s_{m, n}} Z\left(s, \Phi^{n, r}\left(\delta\left(\phi_{1} \otimes \bar{\phi}_{2}\right)\right), f_{1}, f_{2}\right),
$$

where recall that $\Phi^{n, r}\left(\delta\left(\phi_{1} \otimes \bar{\phi}_{2}\right)\right)$ is the Siegel-Weil section associated with $\delta\left(\phi_{1} \otimes \bar{\phi}_{2}\right)$. 
11.6. Local zeta integrals and $L$-factors. For $\operatorname{Re}(s) \gg 0$, if $\Phi=\otimes_{v} \Phi_{v}$ and $f_{i}=\otimes_{v} f_{i, v}$ are pure tensors, one has an Euler product

$$
Z\left(s, \Phi, f_{1}, f_{2}\right)=\prod_{v} Z_{v}\left(s, \Phi_{v}, f_{1, v}, f_{2, v}\right)
$$

where

$$
Z_{v}\left(s, \Phi_{v}, f_{1, v}, f_{2, v}\right)=\int_{G\left(U_{n}\right)\left(F_{v}\right)} \Phi_{v}\left(g_{v}, 1\right) \cdot \overline{\left\langle\pi_{v}\left(g_{v}\right) f_{1}, f_{2}\right\rangle} d g_{v} .
$$

By PS-R], $Z_{v}\left(s, \Phi_{v}, f_{1, v}, f_{2, v}\right)$ extends to a meromorphic function on $\mathbb{C}$. In [LR], Lapid-Rallis has defined the standard $L$-factors $L\left(s, \pi_{v} \times \chi_{V, v}\right)$ using this family of local zeta integrals (see [G] for the metaplectic case). In Y4, Yamana has shown that $L\left(s+\frac{1}{2}, \pi_{v} \times \chi_{V, v}\right)$ is precisely the GCD of the family of doubling zeta integrals associated to "good" sections $\Phi_{v}(s)$. Moreover, when $\operatorname{Re}(s) \geq 0$, a section $\Phi_{v}(s)$ is good at $s$ if and only if it is holomorphic there. Thus, it is natural to define the normalized local zeta integral

$$
Z_{v}^{*}\left(s, \Phi_{v}, f_{1, v}, f_{2, v}\right):=\frac{Z_{v}\left(s, \Phi_{v}, f_{1, v}, f_{2, v}\right)}{L\left(s+\frac{1}{2}, \pi_{v} \times \chi_{V, v}\right)} .
$$

Then $Z_{v}^{*}\left(s, \Phi_{v}, f_{1, v}, f_{2, v}\right)$ is an entire function of $s$ and for any $s_{0}$ such that $\operatorname{Re}\left(s_{0}\right) \geq 0$, one can find a standard section $\Phi$ and $f_{i, v} \in \pi_{v}$ such that

$$
Z_{v}^{*}\left(s_{0}, \Phi_{v}, f_{1, v}, f_{2, v}\right) \neq 0 .
$$

Note that when every data involved is unramified (which is the case for almost all $v$ ), one has

$$
Z_{v}\left(s, \Phi_{v}, f_{1, v}, f_{2, v}\right)=\frac{L\left(s+\frac{1}{2}, \pi_{v} \times \chi_{V, v}\right)}{d_{v}\left(s, \chi_{v}\right)}
$$

where $d_{v}\left(s, \chi_{V}\right)$ is the product of a number of explicit Hecke $L$-factors (see [LR, P. 334, Remark 3]). When $s>0, d_{v}\left(s, \chi_{V}\right)$ has no poles and the Euler product $\prod_{v} d_{v}\left(s, \chi_{V}\right)$ is absolutely convergent, so that

$$
Z^{*}\left(s, \Phi, f_{1}, f_{2}\right):=\prod_{v} Z_{v}^{*}\left(s, \Phi_{v}, f_{1, v}, f_{2, v}\right)
$$

is absolutely convergent (and thus holomorphic) when $\operatorname{Re}(s)>0$.

11.7. Rallis inner product. Hence one has the Rallis inner product formula as follows.

Theorem 11.4. Suppose that $d(n)<m \leq 2 \cdot d(n)$ and $r \leq n$. Let $\pi$ be an irreducible cuspidal representation of $G\left(U_{n}\right)$ and consider its global theta lift $\Theta_{n, r}(\pi)$ on $H\left(V_{r}\right)$. Assume that $\Theta_{n, j}(\pi)=0$ for $j<r$, so that $\Theta_{n, r}(\pi)$ is cuspidal.

(i) For $\phi_{1}, \phi_{2} \in \omega_{\psi, \chi_{V}, V_{r}, U_{n}}$ and $f_{1}, f_{2} \in \pi$, we have

$$
\left\langle\theta\left(\phi_{1}, f_{1}\right), \theta\left(\phi_{2}, f_{2}\right)\right\rangle=[E: F] \cdot \operatorname{Val}_{s=s_{m, n}} L\left(s+\frac{1}{2}, \pi \times \chi_{V}\right) \cdot Z^{*}\left(s, \phi_{1} \otimes \overline{\phi_{2}}, f_{1}, f_{2}\right),
$$

where

$$
s_{m, n}=\frac{m-n-\epsilon_{0}}{2}>0
$$

and $Z^{*}(s,-)$ denotes the normalized doubling zeta integral as in (11.3).

(ii) Assume further that for all places $v$ of $F$, the local theta lift $\Theta_{n, r}\left(\pi_{v}\right)$ is nonzero. Then $L\left(s+\frac{1}{2}, \pi \times \chi_{V}\right)$ is holomorphic at $s=s_{m, n}$, so that in the context of (i),

$$
\left\langle\theta\left(\phi_{1}, f_{1}\right), \theta\left(\phi_{2}, f_{2}\right)\right\rangle=[E: F] \cdot L\left(s_{m, n}+\frac{1}{2}, \pi \times \chi_{V}\right) \cdot Z^{*}\left(s_{m, n}, \phi_{1} \otimes \overline{\phi_{2}}, f_{1}, f_{2}\right) .
$$

In particular, the global theta lift $\Theta_{n, r}(\pi)$ is nonzero if and only if

(a) for all places $v, Z_{v}^{*}\left(s_{m, n}\right)$ is nonzero on $R\left(V_{r, v}\right) \otimes \pi_{v}^{\vee} \otimes \pi_{v}$, and

(b) $L\left(s_{m, n}+\frac{1}{2}, \pi \times \chi_{V}\right) \neq 0$. 
Proof. (i) This follows immediately from Proposition 11.2 together with the definition of $Z^{*}(s,-)$.

(ii) It remains to prove that $L\left(s+\frac{1}{2}, \pi \times \chi_{V}\right)$ is holomorphic at $s=s_{m, n}$. This was essentially shown by Yamana in [Y4, Lemma 10.1], but we give the proof here for the sake of completeness. First assume that $\epsilon_{0} \neq-1$, so $G\left(U_{n}\right)$ is either symplectic or unitary. By [Y4, Theorem $\left.10.1(2)\right]$ and the tower property of global theta lifting, if the $L$-function $L\left(s+\frac{1}{2}, \pi \times \chi_{V}\right)$ is not holomorphic at $s=s_{m, n}$, then there exists an $\epsilon$-Hermitian space $V_{r^{\prime}}^{\#}$ with $\chi_{V_{r^{\prime}}^{\#}}=\chi_{V_{r}}$ and $\operatorname{dim} V_{r}+\operatorname{dim} V_{r^{\prime}}^{\#}=2 \cdot d(n)$ such that the global theta lift $\Theta_{n, V_{r^{\prime}}^{\#}}(\pi)$ to $H\left(V_{r^{\prime}}^{\#}\right)$ is non-vanishing. But since by our assumption $\Theta_{n, j}(\pi)=0$ for all $j<r, V_{r^{\prime}}^{\#}$ belongs to a different Witt tower from $V_{r}$. Hence there is a place $v$ so that $V_{r, v}$ and $V_{r, v}^{\#}$ belong to different Witt towers but nonetheless the two local theta lifts $\Theta_{n, r}\left(\pi_{v}\right)$ and $\Theta_{n, V_{r^{\prime}}^{\#}}\left(\pi_{v}\right)$ to $H\left(V_{r}\right)\left(F_{v}\right)$ and $H\left(V_{r^{\prime}}^{\#}\right)\left(F_{v}\right)$, respectively, are non-vanishing. But the conservation relation (see [KR6], GI2, Thm. 5.4] and [SZ]) then implies that

$$
\operatorname{dim} V_{r, v}+\operatorname{dim} V_{r^{\prime}, v}^{\#} \geq 2 d(n)+2
$$

which contradicts the fact that $V_{r, v}$ and $V_{r^{\prime}, v}^{\#}$ are of complementary dimension.

Next assume $\epsilon_{0}=-1$, so that $G\left(U_{n}\right)$ is orthogonal. Similarly to the above, there exists an automorphic determinant character $\eta$ on $G\left(U_{n}\right)(\mathbb{A})$ such that $\Theta_{n, r^{\prime}}(\pi \otimes \eta) \neq 0$ where $r^{\prime}$ is such that $\operatorname{dim} V_{r}+\operatorname{dim} V_{r^{\prime}}=$ $2 \cdot d(n)$. By the same reasoning as above, this would contradict the conservation relation.

11.8. Local nonvanishing. To obtain the local-global criterion for the nonvanishing of $\Theta_{n, r}(\pi)$ by using the Rallis inner product formula we have obtained, one needs to understand the nonvanishing of local theta lifts in terms of the normalized local zeta integrals. In particular, we hope to have

Conjecture 11.5. The local theta lift $\Theta_{n, r}\left(\pi_{v}\right)$ is nonzero if and only if $Z_{v}^{*}\left(s_{m, n}\right)$ is nonzero on $R\left(V_{r, v}\right) \otimes$ $\pi_{v}^{\vee} \otimes \pi_{v}$ (where $\left.0<s_{m, n} \leq d(n) / 2\right)$.

Note that the implication ( $\Longleftarrow$ ) is obvious, so the content of the conjecture is the reverse implication.

Unfortunately, we are not able to prove this conjecture in full generality when the place $v$ is real. The best we know at this moment is:

Proposition 11.6. Suppose that $0<s_{m, n} \leq \frac{d(n)}{2}$.

(i) Assume that one of the following conditions hold:

- $v$ is finite, or

- $\epsilon_{0}=-1$, or

- $\epsilon_{0}=0, v$ is archimedean and $E_{v}=F_{v} \times F_{v}$, or

- $\epsilon_{0}=1$ and $F_{v}=\mathbb{C}$.

Then the local theta lift $\Theta_{n, r}\left(\pi_{v}\right)$ is nonzero if and only if $Z_{v}^{*}\left(s_{m, n}\right)$ is nonzero on $R\left(V_{r, v}\right) \otimes \pi_{v}^{\vee} \otimes \pi_{v}$.

(ii) Assume that we are in a case not covered by (i), i.e.

- $\epsilon_{0}=0, F_{v}=\mathbb{R}$ and $E_{v}=\mathbb{C}$, or

- $\epsilon_{0}=1, F_{v}=\mathbb{R}$.

Suppose that the signature of $V_{r, v}$ is $(p, q)$ with $p+q=m$. If the local theta lift $\Theta_{n, r}\left(\pi_{v}\right)$ is nonzero, then there is an $\epsilon$-Hermitian space $V_{v}^{\prime}$ over $E_{v}$ such that

(a) the signature $\left(p^{\prime}, q^{\prime}\right)$ of $V_{v}^{\prime}$ (with $p^{\prime}+q^{\prime}=m$ ) satisfies

$$
\begin{cases}p \equiv p^{\prime} & \bmod 4 \text { if } \epsilon_{0}=1 \\ p \equiv p^{\prime} & \bmod 2 \text { if } \epsilon_{0}=0 .\end{cases}
$$


(b) $Z_{v}^{*}\left(s_{m, n}\right)$ is nonzero on $R\left(V_{v}^{\prime}\right) \otimes \pi_{v}^{\vee} \otimes \pi_{v}$;

When $m=d(n)+1$, one has $V_{v}^{\prime}=V_{r, v}$.

Proof. (i) This follows readily from Proposition 5.2 and its archimedean analog using the conservation relation [SZ]; see [Y4, Lemma 8.6].

(ii) This follows from a more detailed knowledge of the module structure of $I_{n}^{n}\left(s_{m, n}, \chi_{V_{r}, v}\right)$. For $s_{m, n} \in$ $X_{n}(\chi)$ and $s_{m, n}>0$, the typical module diagram of $I_{n}^{n}\left(s_{m, n}, \chi_{V_{r}, v}\right)$ is illustrated in [LZ1, Figure 4] if $\epsilon_{0}=0$ and in [LZ2, Figure 5] and [LZ4, $\left.\S 6.1, \S 6.2\right]$ if $\epsilon_{0}=1$. Moreover, in these figures, one may read off the location of the submodules $R_{n}\left(V_{v}\right)$ for all $V_{v}$ such that $\operatorname{dim} V_{v}=\operatorname{dim} V_{r, v}$ and $\chi_{V_{v}}=\chi_{V_{r, v}}$ (if $\epsilon_{0}=1$ ).

Consider the submodule

$$
\Sigma=\bigoplus_{V_{v}^{\prime}} R_{n}\left(V_{v}^{\prime}\right)
$$

where the sum runs over all $\epsilon$-Hermitian spaces $V_{v}^{\prime}$ over $E_{v}$ whose signature $\left(p^{\prime}, q^{\prime}\right)$ satisfies the condition (a) in the Proposition. We need to show that $Z_{v}^{*}\left(s_{m, n}\right)$ is nonzero when restricted to $\Sigma \otimes \pi_{v}^{\vee} \otimes \pi_{v}$.

On examining the module diagram of $I_{n}^{n}\left(s_{m, n}, \chi_{V_{r, v}}\right)$ given in [LZ1, Figure 4], LZ2, Figure 5] and [LZ4, $\S 6.1, \S 6.2]$, one notes that

$$
I_{n}^{n}\left(s_{m, n}, \chi_{V_{r, v}}\right) / \Sigma \cong \bigoplus_{U_{v}} R_{n}\left(U_{v}\right)
$$

where the sum $U_{v}$ runs over all $\epsilon$-Hermitian modules such that

- $\operatorname{dim} U_{v}+\operatorname{dim} V_{r, v}=2 \cdot d(n)$

- $\chi_{U_{v}}=\chi_{V_{r, v}}$ if $\epsilon_{0}=1$;

- $U_{v}$ does not lie in the same Witt tower as the Hermitian spaces $V_{v}^{\prime}$ which occur in the definition of $\Sigma$, and in particular, $U_{v}$ lies in a different Witt tower as $V_{r, v}$.

Since $\Theta_{n, r}\left(\pi_{v}\right)$ is nonzero by hypothesis, the conservation relation shown in SZ implies that the local theta lift of $\pi_{v}$ to any such $H\left(U_{v}\right)$ is zero. Thus $Z_{v}^{*}\left(s_{m, n}\right)$ must be nonzero when restricted to $\Sigma \otimes \pi_{v}^{\vee} \otimes \pi_{v}$.

When $m=d(n)+1$, suppose for the sake of contradiction that $V_{v}^{\prime} \neq V_{r, v}$. Then $V_{v}^{\prime}$ and $V_{r, v}$ belong to two different Witt towers. Since the theta lifts of $\pi_{v}$ to $H\left(V_{r, v}\right)$ and $H\left(V_{v}^{\prime}\right)$ are both nonzero, and the two Witt towers in question are not "adjacent" in the language of [SZ, the conservation relation shown in $[\mathrm{SZ}$ implies that

$$
\operatorname{dim} V_{r, v}+\operatorname{dim} V_{v}^{\prime}>2 d(n)+2 .
$$

This contradicts the fact that $\operatorname{dim} V_{r, v}=\operatorname{dim} V_{v}^{\prime}=d(n)+1$. The Proposition is proved.

11.9. Nonvanishing of theta lifts. Finally, we can state and prove our non-vanishing theorem:

Theorem 11.7. Assume that $d(n)<m \leq 2 \cdot d(n)$ and $r \leq n$. Let $\pi$ be an irreducible cuspidal representation of $G\left(U_{n}\right)$ and consider its global theta lift $\Theta_{n, r}(\pi)$ to $H\left(V_{r}\right)$. Assume that $\Theta_{n, j}(\pi)=0$ for $j<r$, so that $\Theta_{n, r}(\pi)$ is cuspidal.

(i) If $\Theta_{n, r}(\pi)$ is nonzero, then

(a) for all places $v, \Theta_{n, r}\left(\pi_{v}\right) \neq 0$, and

(b) $L\left(s_{m, n}+\frac{1}{2}, \pi \times \chi_{V}\right) \neq 0$, i.e. nonzero holomorphic.

(ii) The converse to (i) holds when one assumes one of the following conditions:

- $\epsilon_{0}=-1$

- $\epsilon_{0}=0$ and $E_{v}=F_{v} \times F_{v}$ for all archimedean places $v$ of $F$;

- $\epsilon_{0}=1$ and $F$ is totally complex; 
- $m=d(n)+1$.

(iii) In general, under the conditions (a) and (b) in (i), there is a $\epsilon$-Hermitian space $V^{\prime}$ over $E$ such that - $V^{\prime} \otimes F_{v} \cong V_{r} \otimes F_{v}$ for every finite or complex place of $F$;

- the global theta lift $\Theta_{U_{n}, V^{\prime}}(\pi)$ of $\pi$ to $H\left(V^{\prime}\right)$ is nonzero.

Under any one of the conditions of (ii), one may take $V^{\prime}$ to be $V_{r}$.

Proof. (i) This follows immediately from Theorem 11.4(ii).

(ii) This follows from Theorem 11.4(ii) and Proposition 11.6.

(iii) For each real place $v$ of $F$ as in Proposition 11.6(ii), pick an $\epsilon$-Hermitian space $V_{v}^{\prime}$ as supplied by Proposition 11.6(ii). The condition on the signature of $V_{v}^{\prime}$ ensures that there is an $\epsilon$-Hermitian space $V^{\prime}$ over $E$ such that $V^{\prime} \otimes_{F} F_{v} \cong V_{v}^{\prime}$ for these places $v$, and $V^{\prime} \otimes_{F} F_{v} \cong V_{r} \otimes_{F} F_{v}$ for all other places. Now one applies Theorem 11.4 with $V^{\prime}$ in place of $V_{r}$ to deduce the desired result.

The reader may recall from $\$ 3.2$ that the condition $r \leq n$ in the above theorem holds automatically, except when $\epsilon_{0}=1$ and $m=2 r=2 n+2$, so that

$$
H\left(V_{r}\right) \cong \mathrm{O}_{n+1, n+1} \text { (split) and } G\left(U_{n}\right)=\mathrm{Sp}_{n}
$$

and $n$ is necessarily even. There is, however, no harm in omitting this case from the discussion. Indeed, the dual pair $\left(G\left(U_{n}\right), H\left(V_{r}\right)\right)$ is in the so-called stable range, so that one knows the global and local theta lifts in question are all nonzero. In fact, even the pair $\left(G\left(U_{n}\right), H\left(V_{r-1}\right)\right)$, which is the lower step of the tower, is in the stable range. Thus, one sees that $\Theta_{n, r-1}(\pi) \neq 0$ and $\Theta_{n, r}(\pi) \neq 0$ is non-cuspidal.

\section{REFERENCES}

[G] W. T. Gan, Doubling zeta integrals and local factors for metaplectic groups, Nagoya Math. Journal 208 (2012), 67-95.

[GI1] W. T. Gan and A. Ichino, On endoscopy and the refined Gross-Prasad conjecture for $\left(\mathrm{SO}_{5}, \mathrm{SO}_{4}\right)$, J. Inst. Math. Jussieu 10 (2011), 235-324.

[GI2] W. T. Gan and A. Ichino, Formal degree and local theta correspondence, to appear in Inventiones Math.

[GS] W. T. Gan and G. Savin, Representations of metaplectic groups I: epsilon dichotomy and local Langlands correspondence, Compositio Math. Vol. 148 (2012), issue 06, pp. 1655-1694.

[GT] W. T. Gan and S. Takeda, On the regularized Siegel-Weil formula (the second term identity) and non-vanishing of theta lifts from orthogonal groups, J. Reine Angew. Math. 659 (2011).

[GJS] D. Ginzburg, D. Jiang and D. Soudry, Poles of L-functions and theta liftings for orthogonal groups, J. Inst. Math. Jussieu 8 (2009), 693-741.

[GJ] R. Godement and H. Jacquet, Zeta functions of simple algebras, Lecture Notes in Mathematics 260, Springer-Verlag, Berlin-New York, 1972.

[Ha] N. Harris, On the refined Gross-Prasad conjecture for unitary groups, Int. Math. Res. Notices (2012) doi: $10.1093 / \mathrm{imrn} / \mathrm{rns} 219$.

[HKS] M. Harris, S. Kudla and J. Sweet, Theta dichotomy for unitary groups, J. Amer. Math. Soc. 9 (1996), 941-1004.

[HLS] M. Harris, J. S. Li and C. Skinner, The Rallis inner product formula and p-adic L-functions, in Automorphic representations, L-functions and applications: progress and prospects, 225-255, Ohio State Univ. Math. Res. Inst. Publ., 11, de Gruyter, Berlin, 2005.

[HL] R. Howe and S. T. Lee, Degenerate principal series representations of $\mathrm{GL}_{n}(\mathbb{C})$ and $\mathrm{GL}_{n}(\mathbb{R})$, J. Funct. Anal. 166 (1999), no. 2, 244-309.

[I1] A. Ichino, On the regularized Siegel-Weil formula, J. Reine Angew. Math. 539 (2001), 201-234.

[I2] A. Ichino, A regularized Siegel-Weil formula for unitary groups, Mathematische Zeitschrift 247 (2004), 241-277.

[I3] A. Ichino, On the Siegel-Weil formula for unitary groups, Math. Z. 255 (2007), no. 4, 721-729.

[Ik] T. Ikeda, On the residue of the Eisenstein series and the Siegel-Weil formula, Compositio Math. 103 (1996), 183-218

[JS] D. H. Jiang and D. Soudry, On the genericity of cuspidal automorphic forms of $S_{2 n+1}$ II, Compositio Math. Volume 143 Part 3 (May 2007), 721-748.

[K1] S. S. Kudla, On the local theta-correspondence, Invent. Math. 83 (1986), 229-255.

[K2] S. Kudla, Splitting metaplectic covers of dual reductive pairs, Israel J. Math. 87 (1994), 361-401. 
[K3] S. Kudla, Some extensions of the Siegel-Weil formula in Eisenstein series and applications, 205-237, Progr. Math., 258, Birkhauser 2008.

[KR1] S. Kudla and S. Rallis, On the Weil-Siegel Formula, Journal Reine Angew. Math. 387 (1988), 1-68.

[KR2] S. Kudla and S. Rallis, On the Weil-Siegel Formula II: Isotropic Convergent Case, J. Reine Angew. Math. 391 (1988), 65-84.

[KR3] S. S. Kudla and S. Rallis, Poles of Eisenstein series and L-functions, Festschrift in honor of I. I. Piatetski-Shapiro on the occasion of his sixtieth birthday, Part II, Israel Math. Conf. Proc. 3, Weizmann, Jerusalem, 1990, 81-110.

[KR4] S. Kudla and S. Rallis, Ramified degenerate principal series representations for $\operatorname{Sp}(n)$, Israel J. Math. 78 (1992), 209-256.

[KR5] S. Kudla and S. Rallis, A regularized Siegel-Weil formula: the first term identity, Ann. Math. 140 (1994), 1-80.

[KR6] S. Kudla and S. Rallis, On first occurrence in the local theta correspondence, Automorphic representations, Lfunctions and applications: progress and prospects, Ohio State Univ. Math. Res. Inst. Publ. 11, de Gruyter, Berlin, 2005, 273-308.

[KRS] S. Kudla, S. Rallis and D. Soudry, On the degree 5 L-function for $\mathrm{Sp}(2)$, Invent. Math. 107 (1992), no. 3, $483-541$.

[KS] S.S.Kudla and W.J. Sweet, Jr., Degenerate principal series representations for U(n,n), Israel J. Math. 98 (1997), 253-306.

[LR] E. Lapid and S. Rallis, On the local factors of representations of classical groups, in Automorphic representations, Lfunctions and applications: progress and prospects, 309-359, Ohio State Univ. Math. Res. Inst. Publ., 11, de Gruyter, Berlin, 2005

[L1] S. T. Lee, On some degenerate principal series representations of $\mathrm{U}(n, n)$ J. Funct. Anal. 126 (1994), no. 2, 305-366.

[L2] S. T. Lee, Degenerate principal series representations of $\operatorname{Sp}(2 n, \mathbb{R})$, Compositio Math. 103 (1996), no. 2, $123-151$.

[LZ1] S. T. Lee and C. B. Zhu, Degenerate principal series and local theta correspondence, Trans. Amer. Math. Soc. 350 (1998), no. 12, 5017-5046.

[LZ2] S. T. Lee and C. B. Zhu, Degenerate principal series and local theta correspondence II, Israel J. Math. 100 (1997), $29-59$.

[LZ3] S. Lee and C. Zhu, Degenerate principal series and local theta correspondence III: The case of complex groups, J. Algebra 319 (2008), no. 1, 336-359.

[LZ4] S. Lee and C. Zhu, Degenerate principal series of metaplectic groups and Howe correspondence, in Automorphic Representations and L-Functions, 379-408, Tata Institute of Fundamental Research, India 2013.

[Li] J. S. Li, Nonvanishing theorems for the cohomology of certain arithmetic quotients, J. Reine Angew. Math. 428 (1992), $177-217$.

[Lo] H. Loke, Howe quotients of unitary characters and unitary lowest weight modules with an appendix by Soo Teck Lee, Represent. Theory 10 (2006), 21-47 (electronic).

[Mo] C. Moeglin, Non nullité de certains relêvements par sêries théta, J. of Lie Theory 7 (1997), 201-229.

[MVW] C. Mœglin, M.-F. Vignéras, and J.-L. Waldspurger, Correspondances de Howe sur un corps p-adique, Lecture Notes in Mathematics 1291, Springer-Verlag, Berlin, 1987.

[PS-R] I. I. Piatetski-Shapiro and S, Rallis, L-functions for classical groups, Part A in Lecture Notes in Math. 1254, Springer-Verlag, New York, 1-52.

[Q] Y. Qiu, Periods of Saito-Kurokawa representations, Int. Math. Res. Notices (online 2013) doi:10.1093/imrn/rnt179.

[R1] S. Rallis, Injectivity properties of liftings associated to Weil representations, Compositio Math. 52 (1984), no. 2, 139-169.

[R2] S. Rallis, On the Howe duality conjecture, Compositio Math. 51 (1984), 333-399.

[R3] S. Rallis, L-functions and the oscillator representation, Lecture Notes in Mathematics, 1245. Springer-Verlag, Berlin, 1987. xvi+239 pp.

[RS1] S. Rallis and G. Schiffman, Automorphic forms constructed from the Weil representation: holomorphic case, Amer. J. Math. 100 (1978), no. 5, 1049-1122.

[RS2] S. Rallis and G. Schiffman, Weil representation. I. Intertwining distributions and discrete spectrum, Mem. Amer. Math. Soc. 25 (1980), no. 231, iii+203 pp.

[RS3] S. Rallis and G. Schiffman, On a relation between $\tilde{S L_{2}}$ cusp forms and cusp forms on tube domains associated to orthogonal groups, Trans. Amer. Math. Soc. 263 (1981), no. 1, 1-58.

[S1] J. Sweet, A computation of the gamma matrix of a family of p-adic zeta integrals, J. Number Theory 55 (1995), $222-260$.

[S2] J. Sweet Functional equations of p-adic zeta integrals and representations of the metaplectic group, preprint.

[SZ] B. Y. Sun and C. B. Zhu, Conservation relations for local theta correspondence, preprint, available at arXiv:1204.2969

[T1] V. Tan, A regularized Siegel-Weil formula on U(2,2) and U(3), Duke Math. J. 94 (1998), no. 2, 341-378.

[T2] V. Tan, Poles of Siegel Eisenstein series on U(n,n), Canad. J. Math. 51 (1999), no. 1, 164-175.

[W] J.-L. Waldspurger, Correspondance de Shimura, J. Math. Pures et Appl. 59 (1980), 1-133.

[We1] A. Weil, Sur la formule de Siegel dans la theorie des groupes classiques, Acta Math. 113 (1965) 1-87. 
[We2] A. Weil Adeles and algebraic groups, with appendices by M. Demazure and Takashi Ono. Progress in Mathematics, 23. Birkhauser, (1982).

[X] W. Xiong, A weak second term identity of the regularized Siegel-Weil formula for unitary groups, to appear in Math. Zeitschrift.

[Y1] S.Yamana, Degenerate principal series representations for quaternionic unitary groups, Israel J. Math. 185 (2011), $77-124$.

[Y2] S. Yamana, On the Siegel-Weil formula: the case of singular forms, Compositio Math. 147 (2011), 1003-1021.

[Y3] S. Yamana, On the Siegel-Weil formula for quaternionic unitary groups, to appear in American J. of Math.

[Y4] S. Yamana, L-functions and theta correspondence for classical groups, to appear in Inventiones Math.

W. T. Gan and Y. Qiu: National University of Singapore, 21 Lower Kent Ridge Road, Singapore, 119077

E-mail address: matgwt@nus.edu.sg

E-mail address: matqy@nus.edu.sg

S. Takeda: Mathematics Department, University of Missouri, Columbia, 202 Math Sciences Building, Columbia, $\mathrm{MO}, 65211$

E-mail address: takedas@missouri.edu 